\title{
AN EQUIVALENCE FOR IRREDUCIBLE PARAMETRIZATION AND ITS APPLICATIONS TO THE DIRECT PROOF OF AN EQUIVALENCE OF THE PUISEUX PAIRS AND THE MULTIPLICITY SEQUENCES FOR IRREDUCIBLE CURVES*
}

\author{
CHUNGHYUK KANG ${ }^{\dagger}$
}

\begin{abstract}
The first aim is to find an equivalence of irreducible plane curve singularities. After then, as an application, the second aim is to prove easily and rigorously an equivalence of the Puiseux pairs (equivalently, the multiplicity and Puiseux exponents) and the multiplicity sequences for irreducibility plane curve singularities inductively, using the $\sigma$-process only. Moreover, as another application, we prove very easily and analytically the Inverse Theorem, that is, the relationship between Puiseux pairs and the reverse Puiseux pairs. Note also that the equivalence of Puiseux pairs and the multiplicity sequences for irreducible plane curve singularities was once proved by this inverse theorem. Rigorously speaking about an equivalence of irreducible parametrization, we may assume that the parametrization of any irreducible plane curve $C$ is given by $y(t)=t^{n}$ and $z(t)=c_{1} t^{k_{1}}+c_{2} t^{k_{2}}+\cdots=c_{1} t^{k_{1}}(1+H(t))$ where $1<n, 1<k_{1}<k_{2}<\cdots$, and the $c_{i}$ are nonzero complex numbers, and $H(t)$ is just the substitution.

If $n \leq k_{1}$, then the irreducible parametrization of the plane curve $C$ is called the Puiseux expansion, and the Puiseux pairs for the given Puiseux expansion of the curve $C$ has been welldefined. If $n>k_{1}$, it is very interesting to define the Puiseux pairs of the Puiseux expansion which is equivalent to the parametrization of the curve $C$, as the Puiseux pairs of $C$, as follows: Let $s$ be the new parameter defined by a conformal mapping of one complex variable $t$ at the origin such that $s(t)=c_{1}{ }^{\frac{1}{k_{1}}} t(1+H(t))^{\frac{1}{k_{1}}}$ with $z(t)=(s(t))^{k_{1}}$ and $s(0)=0$, and let $t=\phi(s)$ be its inverse. Then, the paramtrization defined by $z(\phi(s))=s^{k_{1}}$ and $y(\phi(s))=b_{1} s^{\ell_{1}}+b_{2} s^{\ell_{2}}+\cdots$, where $1<n=\ell_{1}<\ell_{2}<\cdots$, and $k_{1}<n$, and the $b_{i}$ are nonzero complex numbers, being equivalent to the parametrization of the type $(y(t), z(t))$, is the Puiseux expansion with the parameter $s$. In this case, the Puiseux pairs for the curve $C$ can be defined from the Puiseux expansion parametrized by $y_{1}(s)=y(\phi(s))$ and $z_{1}(s)=z(\phi(s))$.

The second aim for this paper is, to prove the following theorem( Theorem $A$ ) in an elementary way, without using the well-known theorem (Theorem $B$ ):

TheOREM A: Whenever any two irreducible parametrizations have the same Puiseux pairs (equivalently, the same multiplicity and Puiseux exponents) by a nonsingular change of the parametrization, then they have the same multiplicity sequences, and conversely.
\end{abstract}

Theorem B: As far as arbitrary Puiseux expansion of irreducible plane curve singularities is concerned, any two irreducible plane curve singularities have the same topological type if and only if they have the same Puiseux pairs.

For example, we can prove by Theorem $A$ that the standard Puiseux expansion defined by $y=t^{n}$ and $z=t^{\alpha_{1}}+\cdots+t^{\alpha_{r}}$, and another parametrization defined by $y=t^{\alpha_{1}}$ and $z=t^{n}\{1+$ $\left.t^{\alpha_{2}-\alpha_{1}}+\cdots+t^{\alpha_{r}-\alpha_{1}}\right\}$ have the same multiplicity sequence, and also the same Puiseux pairs by a nonsingular change of a parameter, without using Theorem $B$.

1. Introduction. The first aim in this paper is to find an equivalence of irreducible plane curve singularities. After then, as an application, the second aim in this paper is to prove easily and rigorously an equivalence of the Puiseux pairs (equivalently, the multiplicity and Puiseux exponents) and the multiplicity sequences for irreducibility plane curve singularities inductively, using the $\sigma$-process only. Moreover, as another application, we prove very easily and analytically the Inverse Theorem,

* Received August 1, 2002; accepted for publication September 8, 2003.

† Department of Mathematics,Seoul National University, Seoul 151-747, Korea (chkang@math.snu.ac.kr). 
that is, the relationship between the Puiseux pairs and the reverse Puiseux pairs. Note also that the equivalence of Puiseux pairs and the multiplicity sequences for irreducible plane curve singularities was already proved in the algebraic form, by this Inverse Theorem.

In this paper, we mention the following well-known theorem (Theorem B), without proof:

Theorem B. As far as arbitrary Puiseux expansion of irreducible plane curve singularities is concerned, any two irreducible plane curve singularities have the same topological types if and only if they have the same type of the standard Puiseux expansion (or the same Puiseux pairs).

(*) Throughout this paper, we will complete our aim without using Theorem B.

Now in more detail, let the parametrization of an irreducible plane curve $C$ be given by $y=t^{n}$ and $z=c_{1} t^{k_{1}}+c_{2} t^{k_{2}}+\cdots=c_{1} t^{k_{1}}(1+H(t))$ where $1<n, 1<k_{1}<k_{2}<$ $\cdots$, and the $c_{i}$ are nonzero complex numbers and $H(t)$ is the just the substitution. If $n \leq k_{1}$, then the above parametrization is called the Puiseux expansion for the curve $C$. In particular, if the parametrization is defined by $y=t^{n}$ and $z=t^{\alpha_{1}}+t^{\alpha_{2}}+\cdots+t^{\alpha_{r}}$ where $2 \leq n<\alpha_{1}<\alpha_{2}<\cdots<\alpha_{r}$ and $n>\operatorname{gcd}\left(n, \alpha_{1}\right)>\operatorname{gcd}\left(n, \alpha_{1}, \alpha_{2}\right)>\cdots>$ $\operatorname{gcd}\left(n, \alpha_{1}, \alpha_{2}, \ldots, \alpha_{r}\right)=1$, then it is called the standard Puiseux expansion for the irreducible plane curve.

In order to avoid the complexity of the terminology in this paper, first of all, we can rewrite the statement of the definition of the Puiseux pairs, as follows.

Definition 1.1. Let the parametrization for arbitrary irreducible plane curve $C$ be defined by

$$
y(t)=t^{n}, \quad z(t)=c_{1} t^{k_{1}}+c_{2} t^{k_{2}}+\cdots=c_{1} t^{k_{1}}(1+H(t)),
$$

where $1<n, 1<k_{1}<k_{2}<\cdots$, and the $c_{i}$ are nonzero complex numbers and $H(t)$ is just the substitution.

Moreover, note that the curve $C$ is irreducible in $\mathbb{C}\{y, z\} \Longleftrightarrow n \geq \operatorname{gcd}\left(n, k_{1}\right) \geq$ $\operatorname{gcd}\left(n, k_{1}, k_{2}\right) \geq \cdots \geq \operatorname{gcd}\left(n, k_{1}, k_{2}, \ldots\right)=1$.

Now, consider two cases, respectively.

Case[I] Let $n \leq k_{1}$. Then, the parametrization for the curve $C$ of (1.0.1) is called the Puisuex expansion.

Case[II] Let $n>k_{1}$. Then, the parametrization for the curve $C$ of (1.0.1) is not called the Puisuex expansion.

Case[I] Assume that $n \leq k_{1}$. Now, we can define the sequence $\left\{\gamma_{1}, \gamma_{2}, \ldots, \gamma_{p}\right\}$ from the set $\left\{k_{i}: i=1,2, \ldots\right\}$, consisting of the exponents of the above parameter $t$, as follows: Note that $n$ is the multiplicity of the curve $C$ at the origin.

$(* *) \gamma_{1}$ is the smallest positive integer among the exponents $k_{i}$ such that $n>\operatorname{gcd}\left(n, \gamma_{1}\right)$; $\gamma_{2}$ is the smallest positive integer among the exponents $k_{i}$ such that $n>\operatorname{gcd}\left(n, \gamma_{1}\right)>$ $\operatorname{gcd}\left(n, \gamma_{1}, k_{i}\right) ; \gamma_{3}$ is the smallest positive integer among the exponents $k_{i}$ such that $n>\operatorname{gcd}\left(n, \gamma_{1}\right)>\operatorname{gcd}\left(n, \gamma_{1}, \gamma_{2}\right)>\operatorname{gcd}\left(n, \gamma_{1}, \gamma_{2}, k_{i}\right) ; \ldots ; \gamma_{p}$ is the smallest positive integer among the exponents $k_{i}$ such that $n>\operatorname{gcd}\left(n, \gamma_{1}\right)>\operatorname{gcd}\left(n, \gamma_{1}, \gamma_{2}\right)>$ $\operatorname{gcd}\left(n, \gamma_{1}, \gamma_{2}, \gamma_{3}\right)>\cdots>\operatorname{gcd}\left(n, \gamma_{1}, \gamma_{2}, \ldots, \gamma_{p}\right)=1$. 
(1) By the uniqueness of construction of the set $\left\{\gamma_{i}: 1 \leq i \leq p\right\}, \gamma_{i}$ is called $i$-th Puiseux exponent in this paper.

(2) By (1), let $\mathrm{S}$ be the set defined by $\left\{n, \gamma_{1}, \gamma_{2}, \ldots, \gamma_{p}\right\}$. Whenever the Puiseux expansion for the curve $C$ is given, then the set $S$ is uniquely determined.

(2a) In this paper, $S$ is called the multiplicity and Puiseux exponents for a given Puiseux expansion of the curve $C$, that is, a new terminology.

(2b) If necessary, we can construct uniquely the standard Puiseux expansion defined by $y=t^{n}$ and $z=t^{\gamma_{1}}+t^{\gamma_{2}}+\cdots+t^{\gamma_{p}}$ for the curve $C$.

(3) By $(2)$, let $d_{i}=\operatorname{gcd}\left(n, \gamma_{1}, \ldots, \gamma_{i}\right)$ for $1 \leq i \leq p$, and write $d_{0}=n$ for brevity of notation.

Define $\lambda_{i}$ and $\mu_{i}$ by $\lambda_{i}=\frac{\gamma_{i}}{d_{i}}$ and $\mu_{i}=\frac{d_{i-1}}{d_{i}}$ for $1 \leq i \leq p$, and let $\left(\lambda_{i}, \mu_{i}\right)$ be defined by the Puiseux pair for each $i$.

Then, $\left\{\left(\lambda_{i}, \mu_{i}\right): i=1,2, \ldots, p\right\}$ is called a finite sequence of Puiseux pairs for a given Puiseux expansion of the curve $C$. If necessary, this sequence will be sometimes called the set of Puiseux pairs for a given Puiseux expansion of the curve $C$.

(4) By the next remark, it can be shown that there is a one-to-one correspondence between the set of the multiplicity and Puiseux exponents, and the set of Puiseux pairs, that is, (2) and (3) have the same type of definitions arithmetically.

(4a) If the parametrization defined by $(y(t), z(t))$ in (1.0.1) is the Puiseux expansion, then it is said that this Puiseux expansion have either the multiplicity sequence and Puiseux exponents $\left\{n, \gamma_{1}, \gamma_{2}, \ldots, \gamma_{p}\right\}$, or the Puiseux pairs $\left\{\left(\lambda_{i}, \mu_{i}\right): i=\right.$ $1,2, \ldots, p\}$ where each $\lambda_{i}$ and $\mu_{i}$ is defined as we have seen in (3).

(5) By (4), throughout this paper, we prefer to choose the terminology in (2) rather than that in $(3)$, if necessary.

Case[II] Assume that $n>k_{1}$. For the convenience of the notation, we may begin without loss of generality that the parametrization of the pair $(y(t), z(t))$ for the curve $C$ of (1.0.1) is written in the following:

$$
y(t)=t^{m}, \quad z(t)=b_{1} t^{\beta_{1}}+b_{2} t^{\beta_{2}}+\cdots, \quad \text { with } m>\beta_{1}
$$

where the $b_{i}$ are nonzero complex numbers, and $m>1$ and $1<\beta_{1}<\beta_{2}<\beta_{3}<\cdots$, and $m \geq \operatorname{gcd}\left(m, \beta_{1}\right) \geq \operatorname{gcd}\left(m, \beta_{1}, \beta_{2}\right) \geq \cdots \geq \operatorname{gcd}\left(n, \beta_{1}, \beta_{2}, \ldots\right)=1$.

By (1.0.2), let $s$ be the new parameter defined by a conformal mapping

$$
s(t)=t\left(b_{1}+\sum_{i \geq 2} b_{i} t^{\beta_{i}-\beta_{1}}\right)^{\frac{1}{\beta_{1}}}
$$

of $t$ at the origin such that $z(s(t))=s^{\beta_{1}}$ and $s(0)=0$, and let $t=\phi(s)$ be its inverse.

Then, the Puiseux expansion defined by $y_{1}(s)=y(\phi(s))$ and $z_{1}(s)=z(\phi(s))$, which is equivalent to the parametrization of the pair $(y(t), z(t))$ in $(1.0 .2)$, can be written as follows:

$$
z_{1}(s)=s^{\beta_{1}}, \quad y_{1}(s)=c_{1} s^{\ell_{1}}+c_{2} s^{\ell_{2}}+\cdots, \quad \text { with } \beta_{1}<\ell_{1}
$$

where $1<m=\ell_{1}<\ell_{2}<\cdots$, and $\beta_{1}<\ell_{1}$, and the $c_{i}$ are nonzero complex numbers.

Therefore, if $m=\ell_{1}$ is greater than $\beta_{1}$, then by using Theorem 3.4 in this paper, first we will find the inverse $t=\phi(s)$ of a conformal mapping $s=s(t)$ in (1.0.2), which gives an algorithm for the construction of the Puiseux expansion in (1.0.4), 
that is, an equivalent parametrization for the above curve $C$. Next, applying the new terminology to this case, by the same way as we have used in Case[I] of this definition, we can naturally generalize the definition of the following words for this curve $C$ of (1.0.4) in Case[II], respectively:

The multiplicity and Puiseux exponents; the standard Puiseux expansion; a finite sequence of the Puiseux pairs:

REMARK 1.1.1. If the multiplicity and Puiseux exponents(equivalently, the standard Puiseux expansion) are given, then it is clear that a finite sequence of the Puiseux pairs is uniquely determined. Conversely, if a finite sequence of the Puiseux pairs is given, then we show that the construction of the multiplicity and the Puiseux exponents is trivial, which can be easily proved from the following computations:

For the proof, follow the same notation as we have seen in both (2) and (3) of Case[I] of Definition 1.1. Since $\left(\lambda_{p}, \mu_{p}\right)$ is given and $d_{p}=1$ from the assumption, then we can compute $\gamma_{p}$ and $d_{p-1}$ because $\lambda_{p}=\gamma_{p}$ and $\mu_{p}=d_{p-1}$. If $p=1$, then $\gamma_{p}$ and $d_{p-1}=n$ were already computed, and so the proof is done. Let $p>1$. Next, since $\left(\lambda_{p-1}, \mu_{p-1}\right)$ is given by assumption, and $\gamma_{p}$ and $d_{p-1}$ were already computed, then we can compute $\gamma_{p-1}$ and $d_{p-2}$ because $\lambda_{p-1}=\frac{\gamma_{p-1}}{d_{p-1}}$ and $\mu_{p-1}=\frac{d_{p-2}}{d_{p-1}}$. If $p=2$, then $\gamma_{p}, \gamma_{p-1}$, and $d_{p-2}=n$ were already computed, and so the proof is done. Let $p>2$. Thus, following the induction method on the positive integer $p$, the proof can be easily done with $d_{0}=n$.

The first aim is to find an algorithm for the construction of an irreducible plane curve $C_{p}$ with the Puiseux expansion which is equivalent to the parametrization of given any irreducible plane curve $C$ of the above type (1.0.1) whether or not $n \leq k_{1}$, by a conformal mapping theorem of one complex variable(Theorem 3.4). As an easy corollary of Theorem 3.4, we can find an easy algorithm for getting the standard Puiseux expansion topologically equivalent to the parametrization of either the curve $C_{p}$ or the curve $C$, by Definition 4.4 and Theorem 4.5.

The second aim is, as an application of Theorem 3.4, to prove easily an equivalence of the Puiseux pairs(equivalently, the multiplicity and the Puiseux exponents) and the multiplicity sequences for irreducible plane curve singularities inductively, using the $\sigma$-process only, without using the well-known theorem (Theorem B), which can be represented as follows:

THEOREM 5.1 (THEOREM A). Whenever any two irreducible parametrizations have the same Puiseux pairs in the sense of Definition 1.1, then they have the same multiplicity sequences, and conversely.

For example, let the parametrization of an irreducible plane curve $C_{1}$ be given by $y(t)=t^{n}$ and $z(t)=c_{1} t^{k_{1}}+c_{2} t^{k_{2}}+\cdots=c_{1} t^{k_{1}}(1+H(t))$ whether or not $n \leq k_{1}$, where $1<n, 1<k_{1}<k_{2}<\cdots$, and the $c_{i}$ are nonzero complex numbers and $H(t)$ is the just the substitution. As compared with the parametrization for the curve $C_{1}$, let $C_{2}$ be the curve parametrized by $y=c_{1} t^{n}(1+H(t))$ and $z=t^{k_{1}}$.

For the proof of the second aim in this example, first it is easy to show by Theorem 3.4 that two curves $C_{1}$ and $C_{2}$ have the same type of the Puiseux pairs in the sense of Definition 1.1, and next we will prove by Theorem 5.1 that they have the same multiplicity sequence, without using Theorem $B$. 
In particular, let the standard Puiseux expansion for the irreducible curve $C_{1}$ be defined by $y=t^{n}$ and $z=t^{\alpha_{1}}+t^{\alpha_{2}}+\cdots+t^{\alpha_{r}}$ where $2 \leq n<\alpha_{1}<\alpha_{2}<\cdots<\alpha_{r}$ and $n>\operatorname{gcd}\left(n, \alpha_{1}\right)>\operatorname{gcd}\left(n, \alpha_{1}, \alpha_{2}\right)>\cdots>\operatorname{gcd}\left(n, \alpha_{1}, \alpha_{2}, \ldots, \alpha_{r}\right)=1$, and let the parametrization of the curve $C_{2}$ be defined by $z=t^{\alpha_{1}}$ and $y=t^{n}+t^{n+\alpha_{2}-\alpha_{1}}+$ $t^{n+\alpha_{3}-\alpha_{1}}+\cdots+t^{n+\alpha_{r}-\alpha_{1}}$. Then, $C_{1}$ and $C_{2}$ have the same type of the Puiseux pairs in the sense of Definition 1.1, and also they have the same multiplicity sequence by Theorem 5.1.

As another application, we can prove very easily and analytically the Inverse Theorem(Theorem 6.2), which has been written in ([Ab3]) without any other proof. Also, this theorem is equivalent to the Inverse Theorem([Ab1]), originally written in the algebraic form, with proof.

As far as irreducible plane curve singularities are concerned, it was just wellknown by Theorem $2.7([\mathrm{En}-\mathrm{Ch}])$ and by $([\mathrm{Br}-\mathrm{Kn}])$ that knowledge of the Puiseux pairs is equivalent to knowledge of the multiplicity sequences. Also, it was proved algebraically by ([Ab1], [Ab2],[Ab3]) that the Puiseux pairs and the multiplicity sequences are equivalent, by using the reverse Puiseux pairs. Now, for example, consider two irreducible plane curves $V=\left\{y=t^{4}\right.$ and $\left.z=t^{6}+t^{9}+t^{10}\right\}$ and $W=$ $\left\{y=t^{4}+t^{7}+t^{8}\right.$ and $\left.z=t^{6}\right\}$ with isolated singularity at the origin, respectively. But, it has been not yet proved rigorously that both $V$ and $W$ have the same Puiseux pairs, and that both $V$ and $W$ have the same multiplicity sequences. So, in this paper we are going to show rigorously that not only $V$ and $W$ have the desired property, but also this result can be generalized, by the conformal mapping theorem of one complex variable and a $\sigma$-process only.

In general, in order to grasp the contents of this paper with ease and simplicity, let $f(y, z)$ be irreducible in $\mathbb{C}\{y, z\}$ with an isolated singularity at the origin in $\mathbb{C}^{2}$ where $\mathbb{C}\{y, z\}$ is the ring of convergent power series at the origin in $\mathbb{C}^{2}$, and $V(f)$ be an analytic variety defined by $f$ at the origin. Then, assume that the curve defined by $f$ at the origin has an irreducible parametrization as follows: For notation of brevity, the curve defined by the above $f$ is also denoted by $V(f)$, if necessary.

$$
y(t)=t^{n}, \quad z(t)=c_{1} t^{k_{1}}+c_{2} t^{k_{2}}+\cdots,
$$

where $1<n, 1<k_{1}<k_{2}<\cdots$, and the $c_{i}$ are nonzero complex numbers, and $n \geq \operatorname{gcd}\left(n, k_{1}\right) \geq \operatorname{gcd}\left(n, k_{1}, k_{2}\right) \geq \cdots \geq \operatorname{gcd}\left(n, k_{1}, k_{2}, \ldots\right)=1$.

To solve the first aim is to construct the irreducible curve $C_{1}$ parametrized by the Puiseux expansion, which is equivalent to the parametrization of $V(f)$ of (1.0.5), whether or not $n \leq k_{1}$.

In preparation for such construction, first of all, it is very interesting and important for us to have the following lemma ( Lemma 1.2), which will be denoted by Lemma 3.3 later.

Lemma 1.2. The irreducible curve $V(f)$ of (1.0.5) can be easily rearranged in the form

$$
\begin{aligned}
y(t)= & t^{n} \\
z(t)= & a_{1} t^{\alpha_{1}}\left(1+D_{1}(t)\right)+a_{2} t^{\alpha_{2}}\left(1+D_{2}(t)\right)+\cdots \\
& +a_{r} t^{\alpha_{r}}\left(1+D_{r}(t)\right)+a_{r+1} t^{\alpha_{r+1}}\left(1+D_{r+1}(t)\right) \\
= & a_{1} t^{\alpha_{1}}(1+H(t))
\end{aligned}
$$


where

(i) $2 \leq n$ and $2 \leq \alpha_{1}=k_{1}<\alpha_{2}<\cdots<\alpha_{r+1}$,

(ii) $n \geq d_{1}>d_{2}>\cdots>d_{r+1}=1$ with $\operatorname{gcd}\left(n, \alpha_{1}, \ldots, \alpha_{i}\right)=d_{i}$ for $1 \leq i \leq r+1$,

(iii) $p_{1}, p_{2}, \ldots, p_{r}$ are nonnegative integers such that $p_{i} d_{i}<\alpha_{i+1}-\alpha_{i}<\left(p_{i}+1\right) d_{i}$ for $1 \leq i \leq r$, $\mathbb{C}\{t\}$,

(iv) $\bar{D}_{j}(t)=\sum_{i=1}^{p_{j}} c_{j i} t^{i d_{j}} \in \mathbb{C}[t]$ for $1 \leq j \leq r$ and $D_{r+1}(t)=\sum_{i=1}^{\infty} c_{j+1, i} t^{i} \in$

(v) $1+H(t)=1+D_{1}(t)+t^{\alpha_{2}-\alpha_{1}}\left(c_{20}+D_{2}(t)\right)+\cdots+t^{\alpha_{r}-\alpha_{1}}\left(c_{r 0}+D_{r}(t)\right)+$ $t^{\alpha_{r+1}-\alpha_{1}}\left(c_{r+1,0}+D_{r+1}(t)\right)$ with $c_{i 0}=\frac{a_{i}}{a_{1}}$ for $2 \leq i \leq r+1$,

(vi) the $a_{i}$ are all nonzero complex numbers with $a_{1}=c_{1}$ for $i=1,2, \ldots, r+1$, from the coefficients $c_{i}$ of (1.0.5).

REMARK 1.2.1. By Lemma 3.3 again, recall that we can define the sequence $\left\{\alpha_{1}, \alpha_{2}, \ldots, \alpha_{r+1}\right\}$ of (1.0.6) from $\left\{k_{i}: i=1,2, \ldots\right\}$ of (1.0.5) by the following way:

Put $\alpha_{1}=k_{1} ; \alpha_{2}$ is the smallest positive integer among the exponents $k_{i}$ such that $n \geq \operatorname{gcd}\left(n, \alpha_{1}\right)>\operatorname{gcd}\left(n, \alpha_{1}, k_{i}\right) ; \alpha_{3}$ is the smallest positive integer among the exponents $k_{i}$ such that $n \geq \operatorname{gcd}\left(n, \alpha_{1}\right)>\operatorname{gcd}\left(n, \alpha_{1}, \alpha_{2}\right)>\operatorname{gcd}\left(n, \alpha_{1}, \alpha_{2}, k_{i}\right)$; $\ldots ; \alpha_{r+1}$ is the smallest positive integer among the exponents $k_{i}$ such that $n \geq$ $\operatorname{gcd}\left(n, \alpha_{1}\right)>\operatorname{gcd}\left(n, \alpha_{1}, \alpha_{2}\right)>\operatorname{gcd}\left(n, \alpha_{1}, \alpha_{2}, \alpha_{3}\right)>\cdots>\operatorname{gcd}\left(n, \alpha_{1}, \alpha_{2}, \ldots, \alpha_{r}\right)>$ $\operatorname{gcd}\left(n, \alpha_{1}, \alpha_{2}, \ldots, \alpha_{r}, k_{i}\right)=1$.

For the construction of the Puiseux expansion which is equivalent to the parametrization of $V(f)$ in (1.0.6), we need to consider two cases: Let $\alpha_{1}=k_{1}$ for notation.

(i) $n \leq \alpha_{1}$ and (ii) $n>\alpha_{1}$.

Case(i) Let $n \leq \alpha_{1}$. Then, the parametrization of $V(f)$ itself is the Puiseux expansion. So, in order to find the curve $C_{1}$ with the standard Puiseux expansion which has the same Puiseux pairs as $V(f)$ of (1.0.6) does, without mentioning the well-known theorem to the parametrization of $V(f)$ in (1.0.6), just apply the definition of the multiplicity and Puiseux exponents in Definition 1.1, to $V(f)$ with the Puiseux expansion, for the following two subcases (ia) and (ib), respectively:

Case(ia): Let $n \leq \alpha_{1}$ and $n>\operatorname{gcd}\left(n, \alpha_{1}\right)$. Then, $n$ is not a divisor of $\alpha_{1}$.

Case(ib): Let $n \leq \alpha_{1}$ and $n=\operatorname{gcd}\left(n, \alpha_{1}\right)$. That is, $n$ is a divisor of $\alpha_{1}$.

Moreover, whether $n$ is a divisor of $\alpha_{1}$ or not, it can be easily proved that $V(f)$ of (1.0.6) and the curve $C_{1}^{*}$ parametrized by $\left(y_{1}^{*}(t), z_{1}^{*}(t)\right)$ have the same Puiseux pairs in the sense of Definition 1.1 where $y_{1}^{*}(t)=t^{n}$ and $z_{1}^{*}(t)=t^{\alpha_{1}}+t^{\alpha_{2}}+\cdots+t^{\alpha_{r+1}}$.

Case(ia) Let $n \leq \alpha_{1}$ and $n>\operatorname{gcd}\left(n, \alpha_{1}\right)$. Now, apply Definition 1.1 to the parametrization of $V(f)$ of (1.0.6). Then, $V(f)$ and the curve $C_{1}=C_{1}^{*}$ parametrized by $\left(y_{1}(t), z_{1}(t)\right)$ have the same Puiseux pairs in the sense of Definition 1.1 where $y_{1}(t)=t^{n}$ and $z_{1}(t)=t^{\alpha_{1}}+t^{\alpha_{2}}+\cdots+t^{\alpha_{r+1}}$ is the standard Puiseux expansion.

Case(ib) Let $n \leq \alpha_{1}$ and $n=\operatorname{gcd}\left(n, \alpha_{1}\right)$. That is, $n$ is a divisor of $\alpha_{1}$. Then, apply Definition 1.1 to the parametrization of $V(f)$ of (1.0.6), too. Then, $V(f)$ and the curve $C_{1}$ parametrized by $\left(y_{1}(t), z_{1}(t)\right)$ have the same Puiseux pairs in the sense of Definition 1.1 where $y_{1}(t)=t^{n}$ and $z_{1}(t)=z_{1}^{*}(t)-t^{\alpha_{1}}=t^{\alpha_{2}}+t^{\alpha_{3}}+\cdots+t^{\alpha_{r+1}}$ is defined to be the standard Puiseux expansion, by Definition 1.1.

But in Case(i), the remaining problem for the the aim of this paper is still to prove that the multiplicity sequences of $V(f)$ and $C_{1}$ are the same(Theorem 5.1). 
Case(ii) Let $n>\alpha_{1}$, and so $n>\operatorname{gcd}\left(n, \alpha_{1}\right)$. First, we must find the method how to construct the irreducible plane curve $C_{p}$ with the Puiseux expansion which is equivalent to the parametrization of the curve $V(f)$ in (1.0.5), for which we need some parameter $s$ with the following properties:

(a) $t=\phi(s)$ is an analytic function of $s$ near $s=0$.

(b) $\phi(0)=0$ and $\phi^{\prime}(0) \neq 0$.

After the curve $C_{p}$ with the above properties (a) and (b) is constructed, just apply the definition of the multiplicity and Puiseux exponents in Definition 1.1, to $C_{p}$ with the Puiseux expansion. Then, it can be easily shown to construct the curve with the standard Puiseux expansion which is equivalent to the parametrization of the curve $C_{p}$, as we have done in Case (i).

But, in order completely to solve the same kind of problem in Case(ii) as we have seen in Case(i), still it remains to show that the multiplicity sequences of $V(f)$ and $C_{p}$ in Case(ii) are the same, but the proof must be the same as that of Case(i), because the multiplicity is invariant under equivalent parametrizations.

Therefore, we can apply Lemma 3.3 and Theorem 3.4 to the parametrization of $V(f)$, so that we may get the Puiseux expansion which is an equivalent parametrization to the curve $V(f)$, whether or not $n \leq k_{1}$.

For Case(ii), let $s$ be the new parameter defined by a conformal mapping of one complex variable $t$ at the origin,

$$
\begin{aligned}
s(t) & =t\left(c_{1}+\sum_{i \geq 2} c_{i} t^{k_{i}-k_{1}}\right)^{\frac{1}{k_{1}}} \\
& =a_{1}^{\frac{1}{\alpha_{1}}} t(1+H(t))^{\frac{1}{\alpha_{1}}} \quad \text { with } s(0)=0, \\
z(s) & =s^{\alpha_{1}}
\end{aligned}
$$

for an equivalent parametrization of the curve $V(f)$ defined by $(y(t), z(t))$ of (1.0.5) or (1.0.6), and let $t=\phi(s)$ be its inverse. Note that $a_{1}=c_{1}$ and $\alpha_{1}=k_{1}$ just for notation.

$(* *)$ Now, to write down the contents of this paper in order is as follows:

(A) For the first aim of this paper, it is just to compute $t=\phi(s)$ in a convergent power series at $s=0$ by Theorem 3.4, and as an easy corollary, to compute $(\phi(s))^{n}$ by using the binomial expansion, for the parametrization $\left(y_{1}(s), z_{1}(s)\right)=$ $(y(\phi(s)), z(\phi(s)))=\left((\phi(s))^{n}, s^{k_{1}}\right)$ in terms of $s$, which is equivalent to the parametrization $(y(t), z(t))$ of $V(f)$ of (1.0.6).

(B) For the curve $V(f)$, it is to find the curve $C_{1}$ with the Puiseux expansion which is equivalent to the parametrization of the curve $V(f)$ by Theorem 4.5 , as an application of $(\mathrm{A})$.

(C) For the second aim of this paper, it is to show by Theorem 3.4 and Theorem 5.1 that the multiplicity and Puiseux exponents(or the Puiseux pairs) and the multiplicity sequences are equivalent, by using a finite sequence of $\sigma$ processes only.

(D) As an application of (A), it is to prove easily and analytically the Inverse Theorem(Theorem 6.2).

In more detail, in order to succeed in the first aim described just as above in this paper, by applying the conformal mapping theorem of one complex variable to $V(f)$ of (1.0.6) in Lemma 1.2, first we can compute $t=\phi(s)$ in $\mathbb{C}\{s\}$ and 
$\left(y_{1}(s), z_{1}(s)\right)=(y(\phi(s), z(\phi(s))$ in the parameter $s$ of $(1.0 .7)$, and then next find an equivalent parametrization for the curve $V(f)$, by the following theorem(Theorem 1.3), which will be denoted by Theorem 3.4, later:

THEOREM 1.3.

Assumption In order to get another parametrization which is equivalent to the pairs $(y(t), z(t))$ of the curve $V(f)$ in either (1.0.5) or (1.0.6), let $t=\phi(s)$ be the inverse function of $s=s(t)$ at the origin, which may be defined by (1.0.7).

Conclusion Then, we have the following consequences [I], [II]:

[I] Then, $t=\phi(s)$ at $s=0$ can be written as follows: Let $\alpha_{1}=k_{1}$.

$$
\begin{aligned}
t= & \phi(s) \\
= & c_{1}^{-\frac{1}{\alpha_{1}}} s\left\{\left(1+Q_{1}(s)\right)+s^{\alpha_{2}-\alpha_{1}}\left(B_{20}+Q_{2}(s)\right)+s^{\alpha_{3}-\alpha_{1}}\left(B_{30}+Q_{3}(s)\right)\right. \\
& \left.+\cdots+s^{\alpha_{r+1}-\alpha_{1}}\left(B_{r+1,0}+Q_{r+1}(s)\right)\right\} \\
= & c_{1}^{-\frac{1}{\alpha_{1}}} s\{1+J(s)\},
\end{aligned}
$$

where

(i) $Q_{j}(s)=\sum_{i=1}^{p_{j}} B_{j i} s^{i d_{j}} \in \mathbb{C}[s]$ with $d_{j}=\operatorname{gcd}\left(n, \alpha_{1}, \ldots, \alpha_{j}\right)$ for $1 \leq j \leq r$ and $Q_{j+1}(s)=\sum_{i=1}^{\infty} B_{r+1, i} s^{i} \in \mathbb{C}\{s\}$,

(ii) all the $B_{j, i(j)}$ are complex numbers with $1 \leq j \leq r+1$ and $1 \leq i(j) \leq p_{j}$, noting that $p_{r+1}$ may be infinite, $r+1$,

(iii) the $B_{j, 0}=\frac{c_{j, 0}}{-\alpha_{1}}\left(c_{1}^{-\frac{1}{\alpha_{1}}}\right)^{\alpha_{j}-\alpha_{1}}$ are all nonzero complex numbers for $2 \leq i \leq$

(iv) $J(s)$ is just the substitution.

[II] As a corollary of $[\mathbf{I}]$ with the binomial series expansion, we get the following equivalent parametization for $V(f)$ of (1.0.6) very easily:

$$
\begin{aligned}
z= & s^{\alpha_{1}} \\
y= & t^{n} \\
= & c_{1}^{-\frac{n}{\alpha_{1}}} s^{n}\left\{\left(1+Q_{1}^{*}(s)\right)+s^{\alpha_{2}-\alpha_{1}}\left(b_{20}+Q_{2}^{*}(s)\right)+s^{\alpha_{3}-\alpha_{1}}\left(b_{30}+Q_{3}^{*}(s)\right)\right. \\
& \left.+\cdots+s^{\alpha_{r+1}-\alpha_{1}}\left(b_{r+1,0}+Q_{r+1}^{*}(s)\right)\right\} \\
= & c_{1}^{-\frac{n}{\alpha_{1}}} s^{n}\{1+L(s)\}
\end{aligned}
$$

where $\mathbb{C}\{s\}$,

(i) $\operatorname{gcd}\left(n, \alpha_{1}, \alpha_{2}-\alpha_{1}, \ldots, \alpha_{i}-\alpha_{1}\right)=\operatorname{gcd}\left(n, \alpha_{1}, \alpha_{2}, \ldots, \alpha_{i}\right)=d_{i}$ for $1 \leq i \leq r+1$,

(ii) $Q_{j}^{*}(s)=\sum_{i=1}^{p_{j}} b_{j i} s^{i d_{j}} \in \mathbb{C}[s]$ for $1 \leq j \leq r$ and $Q_{r+1}^{*}(s)=\sum_{i=1}^{\infty} b_{r+1, i} s^{i} \in$

(iii) all the $b_{j, i(j)}$ are complex numbers with $1 \leq j \leq r+1$ and $1 \leq i(j) \leq p_{j}$, noting that $p_{r+1}$ may be infinite,

(iv*) the $b_{j, 0}$ are all nonzero complex numbers for $2 \leq j \leq r+1$, noting that $b_{j, 0}=n B_{j, 0}$ for $2 \leq j \leq r+1$,

(v) $L(s)$ is just the substitution. 
REMARK 1.3.1. Suppose that the assumption and notations of Theorem 1.3 are satisfied. As a very important application of Theorem 1.3, we have the followings:

(1) Let $C^{*}$ and $C^{* *}$ be two curves defined by the following parametrizations, respectively:

$$
\begin{aligned}
& C^{*}:=\left\{\begin{array}{l}
y=t^{n} \\
z=t^{\alpha_{1}}+t^{\alpha_{2}}+\cdots+t^{\alpha_{r+1}},
\end{array}\right. \\
& C^{* *}:=\left\{\begin{array}{l}
y=t^{\alpha_{1}} \\
z=t^{n}+t^{n+\alpha_{2}-\alpha_{1}}+t^{n+\alpha_{3}-\alpha_{1}}+\cdots+t^{n+\alpha_{r+1}-\alpha_{1}},
\end{array}\right.
\end{aligned}
$$

where

(i) $2 \leq n$ and $2 \leq \alpha_{1}=k_{1}<\alpha_{2}<\cdots<\alpha_{r+1}$,

(ii) $n \geq d_{1}>d_{2}>\cdots>d_{r+1}=1$ with $\operatorname{gcd}\left(n, \alpha_{1}, \ldots, \alpha_{i}\right)=d_{i}$ for $1 \leq i \leq r+1$. Then, apply Theorem 1.3 to the parametrization of $V(f)$, and then it can be easily shown by Lemma 1.2, Theorem 1.3 and Definition 1.1 that $V(f), C^{*}$ and $C^{* *}$ have the same type of the standard Puiseux expansion whether or not $n \leq k_{1}=\alpha_{1}$, because whenever the $a_{i}$ are all nonzero complex numbers with $a_{1}=c_{1}$ for $i=1,2, \ldots, r+1$, then the $b_{j, 0}$ are all nonzero complex numbers for $2 \leq j \leq r+1$, and conversely.

(2) Also, for convenience of notations, it is interesting to note that the parametrization for $V(f)$ of (1.0.6) can be just rewritten as follows: For brevity of notation, write $C=V(f)$.

$$
\begin{aligned}
y= & t^{n} \\
z= & a_{1} t^{\alpha_{1}}\left\{\left(1+D_{1}(t)\right)+t^{\alpha_{2}-\alpha_{1}}\left(\frac{a_{2}}{a_{1}}+\frac{a_{2}}{a_{1}} D_{2}(t)\right)+\cdots\right. \\
& \left.+t^{\alpha_{r}-\alpha_{1}}\left(\frac{a_{r}}{a_{1}}+\frac{a_{r}}{a_{1}} D_{r}(t)\right)+t^{\alpha_{r+1}-\alpha_{1}}\left(\frac{a_{r+1}}{a_{1}}+\frac{a_{r+1}}{a_{1}} D_{r+1}(t)\right)\right\} \\
= & a_{1} t^{\alpha_{1}}(1+H(t)),
\end{aligned}
$$

where $H(t)$ is just the substitution.

Now, in order to compare $L(s)$ of (1.0.9) and $H(t)$ of (1.0.11) simultaneously, let $C$ of (1.0.11) and $\tilde{C}$ be two irreducible curves defined by (i) and (ii) respectively, as follows:

$$
\begin{aligned}
& \text { (i) } y=t^{n} \quad \text { and } z=a_{1} t^{\alpha_{1}}(1+H(t)) . \\
& \text { (ii) } y=t^{n}(1+H(t)) \text { and } z=a_{1} t^{\alpha_{1}} .
\end{aligned}
$$

Even if $H(t)$ may be assumed to be chosen arbitrary, then by Definition 1.1, Theorem 3.4 and Lemma 4.3, two irreducible curves defined by $C$ of (i) and $\tilde{C}$ of (ii) in (1.0.12) have the same Puiseux pairs.

For example, if the standard Puiseux expansion for an irreducible plane curve $C$ is defined by $y=t^{n}$ and $z=t^{\alpha_{1}}(1+H(t))=t^{\alpha_{1}}\left(1+t^{\alpha_{2}-\alpha_{1}}+t^{\alpha_{3}-\alpha_{1}}+\cdots+t^{\alpha_{r}-\alpha_{1}}\right)=$ $t^{\alpha_{1}}+t^{\alpha_{2}}+\cdots+t^{\alpha_{r}}$ where $2 \leq n<\alpha_{1}<\alpha_{2}<\cdots<\alpha_{r}$ and $n>\operatorname{gcd}\left(n, \alpha_{1}\right)>$ $\operatorname{gcd}\left(n, \alpha_{1}, \alpha_{2}\right)>\cdots>\operatorname{gcd}\left(n, \alpha_{1}, \alpha_{2}, \ldots, \alpha_{r}\right)=1$, and the parametrization for an irreducible curve $\tilde{C}$ is defined by $z=t^{\alpha_{1}}$ and $y=t^{n}(1+H(t))=t^{n}\left(1+t^{\alpha_{2}-\alpha_{1}}+\right.$ $\left.t^{\alpha_{3}-\alpha_{1}}+\cdots+t^{\alpha_{r}-\alpha_{1}}\right)$, then $C$ and $\tilde{C}$ have the same Puiseux pairs. 
As a very important application of Theorem 3.4, in order to explain the contents of Theorem 5.1 , let $g(y, z)$ be arbitrary in $\mathbb{C}\{y, z\}$ with an isolated singularity at the origin in $\mathbb{C}^{2}$, and $V(g)$ be an analytic variety defined by $g(y, z)$ at the origin. Then, as we have seen in (1.0.6), it is also important to consider that the parametrization of another irreducible curve $V(g)$ with an isolated singularity at the origin can be given as follows:

$$
\begin{aligned}
y= & t^{m} \\
z= & t^{\beta_{1}}\left(1+L_{1}(t)\right)+b_{2} t^{\beta_{2}}\left(1+L_{2}(t)\right) \\
& +\cdots+b_{u} t^{\beta_{u}}\left(1+L_{u}(t)\right)+b_{u+1} t^{\beta_{u+1}}\left(1+L_{u+1}(t)\right),
\end{aligned}
$$

where

(i) $2 \leq m$ and $2 \leq \beta_{1}<\beta_{2}<\cdots<\beta_{u+1}$,

(ii) $m \geq e_{1}>e_{2}>\cdots>e_{u+1}=1$ with $\operatorname{gcd}\left(m, \beta_{1}, \ldots, \beta_{i}\right)=e_{i}$ for $1 \leq i \leq u+1$,

(iii) $q_{1}, q_{2}, \ldots, q_{u+1}$ are nonnegative integers such that $q_{i} e_{i}<\beta_{i+1}-\beta_{i}<\left(q_{i}+1\right) e_{i}$ for $1 \leq i \leq u$, $\mathbb{C}\{t\}$,

(iv) $\bar{L}_{j}(t)=\sum_{i=1}^{q_{j}} b_{j i} t^{i e_{j}} \in \mathbb{C}[t]$ for $1 \leq j \leq u$ and $L_{u+1}(t)=\sum_{i=1}^{\infty} b_{u+1, i} t^{i} \in$

(v) the $b_{i}$ are all nonzero numbers for $1 \leq i \leq u+1$.

Finally, by applying (1.0.12) and Lemma 4.3 to either (1.0.6) or (1.0.8), and (1.0.13), with a finite $\sigma$-processes, this paper for the second aim may be very easily described by [I], [II], [III] and [IV] as follows:

[I] Assume that $n<\alpha_{1}$ and $n>\operatorname{gcd}\left(n, \alpha_{1}\right)$ in $(1.0,6)$, and also that $m<\beta_{1}$ and $m>\operatorname{gcd}\left(m, \beta_{1}\right)$ in (1.0.13). Note that the parametrizations of both $V(f)$ Of (1.0.6) and $V(g)$ of (1.0.13) are Puiseux expansions. Then, we can prove the following(Theorem5.1).

$V(f)$ and $V(g)$ have the same multiplicity sequence.

$\Longleftrightarrow$ The multiplicity and Puiseux exponents for both $V(f)$ and $V(g)$ are the same, in the sense of Definition 4.1.

That is, $n=m, r+1=u+1$, and $\alpha_{i}=\beta_{i} \quad$ for all $i=1,2, \ldots, r+1$.

$\Longleftrightarrow$ The Puiseux pairs for both $V(f)$ and $V(g)$ are the same, by Definition 4.1.

[II] Assume that $n<\alpha_{1}$ and $n>\operatorname{gcd}\left(n, \alpha_{1}\right)$ in (1.0.6), and that $2 \leq \beta_{1}<$ $m$ and $\beta_{1}<\beta_{2}<\cdots<\beta_{u+1}$ and $\beta_{1}>\operatorname{gcd}\left(m, \beta_{1}\right)>\operatorname{gcd}\left(m, \beta_{1}, \beta_{2}\right)>\cdots>$ $\operatorname{gcd}\left(m, \beta_{1}, \beta_{2}, \ldots, \beta_{u+1}\right)=1$ in (1.0.13). Note that the parametrization of $V(f)$ is the Puiseux expansion, but the parametrization of $V(g)$ is not the Puiseux expansion. So, using the construction method in Theorem 3.4 , let $C_{2}$ be the curve with the Puiseux expansion which is equivalent to the parametrization of $V(g)$ in (1.0.13). Thus, we can get the following by $[\mathrm{I}]$.

$$
\begin{gathered}
V(f) \text { and } C_{2} \text { have the same multiplicity sequence } \\
\Longleftrightarrow n=\beta_{1}, \alpha_{1}=m, r=u \text { and } \alpha_{i}=m+\beta_{i}-\beta_{1} \text { for } 1 \leq i \leq r+1,
\end{gathered}
$$

which is viewed as a necessary and sufficient condition that $V(f)$ and $V(g)$ have the same Puiseux pairs. 
[III] Assume that $n<\alpha_{1}$ and $n>\operatorname{gcd}\left(n, \alpha_{1}\right)$ in (1.0.6), and that $2 \leq \beta_{1}<$ $m$ and $\beta_{1}<\beta_{2}<\cdots<\beta_{u+1}$ and $\beta_{1} \geq \operatorname{gcd}\left(m, \beta_{1}\right)>\operatorname{gcd}\left(m, \beta_{1}, \beta_{2}\right)>\cdots>$ $\operatorname{gcd}\left(m, \beta_{1}, \beta_{2}, \ldots, \beta_{u+1}\right)=1$ with $\beta_{1}=\operatorname{gcd}\left(m, \beta_{1}\right)$. Note that $V(f)$ is the Puiseux expansion, but $V(g)$ is not the Puiseux expansion. By the same technique as in Theorem 3.4, let $C_{2}$ be the curve defined by the Puiseux expansion which is equivalent to the parametrization of $V(g)$ in (1.0.13), and then apply Lemma 4.3 to the curve $C_{2}$ because $\beta_{1}$ is a divisor of $m$. Thus, we can get the following by [I].

$V(f)$ and $C_{2}$ have the same multiplicity sequence.

$\Longleftrightarrow n=\beta_{1}, \alpha_{1}=m+\beta_{2}-\beta_{1}, \alpha_{2}=m+\beta_{3}-\beta_{1}, \ldots, \alpha_{r+1}=m+\beta_{r+2}-\beta_{1}$, where $r+2=u+1$,

which is viewed as a necessary and sufficient condition that $V(f)$ and $C_{2}$ have the same Puiseux pairs. Thus, $V(f)$ and $V(g)$ have the same Puiseux pairs.

[IV] Assume that $n>\alpha_{1}$ and $\alpha_{1}>\operatorname{gcd}\left(n, \alpha_{1}\right)$ in (1.0.6), and that $2 \leq \beta_{1}<$ $m$ and $\beta_{1}<\beta_{2}<\cdots<\beta_{u+1}$ and $\beta_{1}>\operatorname{gcd}\left(m, \beta_{1}\right)>\operatorname{gcd}\left(m, \beta_{1}, \beta_{2}\right)>\cdots>$ $\operatorname{gcd}\left(m, \beta_{1}, \beta_{2}, \ldots, \beta_{u+1}\right)=1$ in (1.0.13). Note that neither $V(f)$ nor $V(g)$ is the Puiseux expansion. But, using the construction method in Theorem 3.4, we may assume that $C_{1}$ is the curve with the Puiseux expansions which is an equivalent parametrization for $V(f)$, and also $C_{2}$ is the curve with the Puiseux expansions which is an equivalent parametrization for $V(g)$. Thus, we can prove the following by [I].

$C_{1}$ and $C_{2}$ have the same multiplicity sequence.

$\Longleftrightarrow \alpha_{1}=\beta_{1}, n=m, r=u$ and $n+\alpha_{i}-\alpha_{1}=m+\beta_{i}-\beta_{1}$ for $1 \leq i \leq r+1$.

That is, $n=m, r=u$ and $\alpha_{i}=\beta_{i}$ for $1 \leq i \leq r+1$.

$\Longleftrightarrow C_{1}$ and $C_{2}$ have the same Puiseux pairs,

which is viewed as a necessary and sufficient condition that $V(f)$ and $V(g)$ have the same Puiseux pairs.

Therefore, summarizing the above results again, then we can prove Theorem 5.1. Moreover, we can prove by Theorem 5.1 and Theorem 4.5 that any two irreducible plane curve singularities have the same topological type of singularities at the origin if and only if they have the same multiplicity sequences. Also, as an application, it is easily computable by Theorem 3.4 that two irreducible curves $V=\left\{y=t^{4}\right.$ and $z=$ $\left.t^{6}+t^{9}\right\}$ and $U_{\lambda}=\left\{y=t^{4}+\lambda t^{7}\right.$ and $\left.z=t^{6}+t^{9}\right\}$ with isolated singularity at the origin have the same multiplicity sequence if and only if $\lambda \neq \frac{2}{3}$.

Finally, using Theorem 3.4, we will prove very easily the Inverse Theorem (Theorem 6.2 , which has been analytically written in ([Ab3]) without proof. This theorem is the restatement of the Inverse Theorem in ([Ab1]), which was already represented and proved in the algebraic statement.

2. Known preliminaries. Let $\mathbb{C}\{y, z\}$ be the ring of convergent power series at the origin in $\mathbb{C}^{2}$. Let $V(f)=\{(y, z): f(y, z)=0\}$ be an analytic variety at $(y, z)=(0,0)$ in $\mathbb{C}^{2}$ with an isolated singular point at the origin where $f$ is in $\mathbb{C}\{y, z\}$ and square-free. Let $\pi: M \rightarrow \mathbb{C}^{2}$ be a blow-up of $\mathbb{C}^{2}$ at $(0,0)$. Let $(v, u)$ and $\left(v^{\prime}, u^{\prime}\right)$ be the local coordinates for $\mathrm{M}$ with $\pi(v, u)=(y, z)=(v u, v)$ and $\pi\left(v^{\prime}, u^{\prime}\right)=$ 
$(y, z)=\left(v^{\prime}, v^{\prime} u^{\prime}\right)$ where $u^{\prime}=\frac{1}{u}$ and $v^{\prime}=v u$. Let $e$ be the multiplicity of $f$ at $(0,0)$ with $e \geq 2$. Then $\pi^{-1}(V(f))$, the total transform of $V(f)$, is locally given by $f(v, v u)=v^{e} f_{1}(v, u)$ and $f\left(v^{\prime} u^{\prime}, v^{\prime}\right)=v^{\prime} f_{2}\left(v^{\prime}, u^{\prime}\right)$. Let $V^{(1)}(f)$ be an analytic variety locally defined by either $f_{1}(v, u)=0$ or $f_{2}\left(v^{\prime}, u^{\prime}\right)=0$. Then, we call $V^{(1)}(f)$ the proper transform of $V(f)$ at $(0,0)$. Note that if $f$ is irreducible in $\mathbb{C}\{y, z\}$, then just one of the local coordinates is needed for the study of $V^{(1)}(f)$. After $m$ iterations of blow-ups, let $\tau_{m}=\pi \circ \pi_{2} \circ \cdots \circ \pi_{m}: M^{(m)} \rightarrow \mathbb{C}^{(2)}$. Let $V^{(m)}(f)$ be the proper transform of $V(f)$ under $\tau_{m}$. Let $E^{(m)}=\tau_{m}^{-1}(0,0)$. Then, $E^{(m)}$ is, by definition, an exceptional set of the first kind. Let $E^{(m)}=\cup_{i=1}^{m} E_{i}$ be the decomposition of $E^{(m)}$ into irreducible components. Each $E_{i}$ is called an exceptional curve of the first kind. Let $\left(f \circ \tau_{m}\right)_{\text {divisor }}=V^{(m)}(f)+\sum_{i=1}^{m} e_{i} E_{i}$ be the divisor of $f \circ \tau_{m}$. Then, we have the following well-known theorem.

THEOREM 2.1. Let $V(f)=\{(y, z): f(y, z)=0\}$ be an analytic variety at $(y, z)=$ $(0,0)$ in $\mathbb{C}^{2}$ with an isolated singular point at the origin where $f$ is in $\mathbb{C}\{y, z\}$ and square-free. There exists an analytic manifold $M$ by the composition of a finite number $(m)$ successive blow-ups, $\tau_{m}: M \rightarrow \mathbb{C}^{2}$, such that if $R$ is the set of regular points on $V$ then $\tau: \overline{\tau_{m}^{-1}(R)} \rightarrow V$ is a resolution of the singular point $(0,0)$ of $V$, where $\overline{\tau_{m}^{-1}(R)}$ is the closure of $\tau_{m}^{-1}(R)$ in $R$.

COROLlARY 2.2. Under the same assumption of Theorem 2.1, after additional blow-ups any two components of $V^{(m)}$ and $\cup_{i=1}^{m} E_{i}$ meet with normal crossings whenever they meet and no three components of $V^{(m)}$ and $E_{i}$ meet, where $V^{(m)}$ and $\cup E_{i}$ are defined just before Theorem 2.1.

\section{DEFINITION 2.3.}

(i) For an isolated singularity of a plane curve, the smallest resolution with normal crossings in the sense of the above corollary is called the standard resolution of the given singularity.

(ii) Let $V(f)=\{(y, z): f(y, z)=0\}$ and $V(g)=\{(y, z): g(y, z)=0\}$ be analytic varieties at $(y, z)=(0,0)$ in $\mathbb{C}^{2}$ with an isolated singular point at the origin where $f$ and $g$ are in $\mathbb{C}\{y, z\}$ and square-free. $V(f)$ and $V(g)$ are said to either have a homeomorphic resolution or be equisingular if $\left(f \circ \tau_{m}\right)_{\text {divisor }}$ and $\left(g \circ \tau_{m}\right)_{\text {divisor }}$ are equivalent in the sense of the standard resolution.

Now, we want to write the local defining equation for singularities of irreducible plane curves in terms of parametrization, if necessary and consider the definition of the multiplicity sequence.

THEOREM 2.4. Suppose that the parametrization for the curve $C$ is given as follows:

$$
y=t^{n} \text { and } z=c_{1} t^{k_{1}}+c_{2} t^{k_{2}}+\cdots
$$

where the $c_{i}$ are nonzero complex numbers, and $0<n, 0<k_{1}<k_{2}<\cdots$. It is not assumed that $n<k_{1}$.

Then the parametrization is irreducible in $\mathbb{C}\{y, z\}$ if and only if $n \geq \operatorname{gcd}\left(n, k_{1}\right) \geq$ $\operatorname{gcd}\left(n, k_{1}, k_{2}\right) \geq \cdots \geq \operatorname{gcd}\left(n, k_{1}, k_{2}, \ldots\right)=1$. 
Definition 2.5. Let $f(y, z)$ be irreducible in $\mathbb{C}\{y, z\}$ with an isolated singularity at the origin. Let the curve $C$ defined by $f(y, z) \in \mathbb{C}\{y, z\}$ have a parametrization with a parameter $t$ as follows:

$$
y=t^{n} \text { and } z=c_{1} t^{k_{1}}+c_{2} t^{k_{2}}+\cdots
$$

where the $c_{i}$ are nonzero complex numbers and $1<n, 1<k_{1}<k_{2}<\cdots$. It is not assumed that $k_{1} \geq n$. Then, we have the following definitions.

(i) If $k_{1} \geq n$, then the above parametrization is called the Puiseux expansion for the curve $C$.

(ii) In particular, if the above parametrization is defined by $y=t^{n}$ and $z=$ $t^{\alpha_{1}}+t^{\alpha_{2}}+\cdots+t^{\alpha_{r}}$ where $2 \leq n<\alpha_{1}<\alpha_{2}<\cdots<\alpha_{r}$ and $n>\operatorname{gcd}\left(n, \alpha_{1}\right)>$ $\operatorname{gcd}\left(n, \alpha_{1}, \alpha_{2}\right)>\cdots>\operatorname{gcd}\left(n, \alpha_{1}, \alpha_{2}, \ldots, \alpha_{r}\right)=1$, then it is called the standard Puiseux expansion, and also by Definition 4.2 in this paper, the set $\left\{n, \alpha_{1}, \alpha_{2}, \ldots, \alpha_{r}\right\}$ will be defined to be the multiplicity and Puiseux exponents for the standard Puiseux expansion by Definition 4.1, as we have seen in Definition 1.1.

DEFINITION 2.6. Let us just consider the definition of the multiplicity sequence for irreducible curves. Suppose that we are given an irreducible curve germ with an isolated singularity. Let $\nu_{0}$ be the multiplicity of this curve germ at this point. If we blow up once, then we again find at most one singularity. Let $\nu_{1}$ be the multiplicity of the curve of the germ blown up once, $\nu_{2}$ be the multiplicity of the curve of the germ blown up twice, and continue to the standard resolution. The sequence ends with a sequences of ends. The sequences of these multiplicities, $\left\{\nu_{1}, \nu_{2}, \ldots, \nu_{n-1}\right\}$, where the last one is not is not counted, is then the multiplicity sequence.

THEOREM 2.7(ENRIQUES-CHISINI).

(i) For an irreducible curve with Puiseux expansion

$$
\begin{aligned}
& x=t^{m} \\
& y=a_{1} t^{k_{1}}+a_{2} t^{k_{2}}+\cdots+a_{q} t^{k_{q}},
\end{aligned}
$$

in which only essential (characteristic) term appear, the multiplicity sequence is determined by the following chain of $g$ Euclidean algorithms: Let $i=1,2, \ldots$.

$$
\begin{aligned}
\lambda_{i} & =\mu_{i, 1} r_{i, 1}+r_{i, 2}, \\
r_{i, 1} & =\mu_{i, 2} r_{i, 2}+r_{i, 3}, \\
& \ldots \ldots \\
r_{i, w(i)-1} & =\mu_{i, w(i)} r_{i, w(i)} \quad \text { with } \quad 0 \leq r_{i, j+1}<r_{i, j}, \\
\lambda_{i} & =k_{i}-k_{i-1} \quad \text { for } 1 \leq i \leq g, \quad \text { and } \quad k_{0}=0, \\
r_{i, 1} & =r_{i-1, w(i-1)} \quad \text { for } i>1, \quad \text { and } \quad r_{1,1}=m .
\end{aligned}
$$

In the multiplicity sequence, the multiplicity $r_{i j}$ then appears $\mu_{i j}$ times, where $i=$ $1, \ldots, g ; j=1, \ldots, w(i)$. (If a certain multiplicity arises from several successive algorithms, then it is also counted multiply.)

(ii) For an arbitrary irreducible curve one obtains the multiplicity sequences by omitting all non-characteristic terms from the Puiseux expansion and then applying the algorithm above. 
(iii) Conversely, one can reconstruct the exponents of the characteristic terms of the Puiseux expansion of an irreducible curve, i.e. the Puiseux pairs of the curve, from the multiplicity sequence, by the chain of Euclidean algorithms.

Proof of Theorem 2.7. See [Bri-Kn].

Definition 2.8. Let $V=\left\{z \in \mathbb{C}^{n+1}: f(z)=0\right\}$ and $W=\left\{z \in \mathbb{C}^{n+1}: g(z)=0\right\}$ be germs of complex analytic hypersurface with isolated singularity at the origin.

(i) $f$ and $g$ are said to have the same topological type of the singularity at the origin if there is a germ at the origin of homeomorphisms $\phi:\left(U_{1}, 0\right) \rightarrow\left(U_{2}, 0\right)$ such that $\phi(V)=W$ and $\phi(0)=0$ where $U_{1}$ and $U_{2}$ are open subsets in $\mathbb{C}^{n+1}$. In this case, denote this relation by $f \sim g$ or $V \sim W$. Otherwise, we write $f \not g$ or $V \not W$.

(ii) $f$ and $g$ are said to have the same analytic type of the singularity at the origin if there is a germ at the origin of biholomorphisms $\psi:\left(U_{1}, 0\right) \rightarrow\left(U_{2}, 0\right)$ such that $\psi(V)=W$ and $\psi(0)=0$ where $U_{1}$ and $U_{2}$ are open subsets in $\mathbb{C}^{n+1}$, that is, $f \circ \psi=u g$ where $u$ is a unit in ${ }_{n+1} \mathcal{O}$, the ring of germs of holomorphic functions at the origin in $\mathbb{C}^{n+1}$. In this case, denote this relation by $f \approx g$ or $V \approx W$. Otherwise, we write $f \not \approx g$ or $V \not \approx W$.

THEOREM 2.9([BR],[Bu],[Z1]). Let $f(y, z)$ be irreducible in $\mathbb{C}\{y, z\}$ with an isolated singularity at the origin in $\mathbb{C}^{2}$. Then the curve defined by $f$ at the origin can be described topologically by $y=t^{n}$ and $z=t^{\alpha_{1}}+t^{\alpha_{2}}+\cdots+t^{\alpha_{p}}$ where $n<\alpha_{1}<\cdots<\alpha_{p}$ and $n>\operatorname{gcd}\left(n, \alpha_{1}\right)>\cdots>\operatorname{gcd}\left(n_{1}, \alpha_{1}, \ldots, \alpha_{p}\right)=1$. If for a given $f$ there is another homeomorphic parametrization defined by $y=t^{m}$ and $z=t^{\beta_{1}}+\cdots+t^{\beta_{q}}$ where $m<\beta_{1}<\cdots<\beta_{q}$ and $m>\operatorname{gcd}\left(m, \beta_{1}\right)>\cdots>\operatorname{gcd}\left(m, \beta_{1}, \ldots, \beta_{q}\right)=1$, then $n=m$, and $p=q$ and $\alpha_{i}=\beta_{i}$ for $1 \leq i \leq p$.

Note by Definition 2.5 that the multiplicity and Puiseux exponents for the standard Puiseux expansion determine the topological types of the irreducible plane curve singularities, and conversely.

3. How to get an equivalent parametrization from any given irreducible parametrization by the inverse mapping theorem of one complex variable.

Definition 3.1. Let $C$ be an irreducible curve defined by the following pair $(y(t), z(t))$ :

$$
\begin{aligned}
& y=a_{1} t^{n_{1}}+a_{2} t^{n_{2}}+\cdots \\
& z=b_{1} t^{k_{1}}+b_{2} t^{k_{2}}+\cdots
\end{aligned}
$$

where $y(t)$ and $z(t)$ are in $\mathbb{C}\{t\}$, and the $a_{i}$ and the $b_{i}$ are nonzero complex numbers, and $0<n_{1}<n_{2}<\cdots$ and $0<k_{1}<k_{2}<\cdots$. Then, the pair $\left(y_{1}(t), z_{1}(t)\right)$ is called an equivalent irreducible parametrization of the pair $(y(t), z(t))$ if there is an analytic function $\phi(t)$ in a neighborhood of zero such that $\phi(0)=0$ and $\phi^{\prime}(0) \neq 0$ and that $y_{1}(t)=y(\phi(t))$ and $z_{1}(t)=z(\phi(t))$.

For example, let $s$ be the new parameter defined by a conformal mapping

$$
s(t)=t\left(b_{1}+\sum_{i \geq 2} b_{i} t^{k_{i}-k_{1}}\right)^{\frac{1}{k_{1}}}
$$

of $t$ at the origin such that $z(s)=s^{k_{1}}$ and $s(0)=0$ and $t=\phi(s)$ be its inverse. Then, an equivalent parametrization of the above curve $C$ defined by $y_{1}(s)=y(\phi(s))$ and 
$z_{1}(s)=z(\phi(s))$ is that $z_{1}=s^{k_{1}}$ and $y_{1}=c_{1} s^{\gamma_{1}}+c_{2} s^{\gamma_{2}}+\cdots$ where $1<\gamma_{1}=n_{1}<$ $\gamma_{2}<\cdots$, and the $c_{i}$ and $b_{1}$ are nonzero numbers.

Problem. We may begin without loss of generality that the parametrization of the pair $(y(t), z(t))$ for the curve $C$ of Definition 3.1 is given by the followings:

$$
\begin{aligned}
& y=t^{n} \\
& z=b_{1} t^{k_{1}}+b_{2} t^{k_{2}}+\cdots
\end{aligned}
$$

where the $b_{i}$ are nonzero complex numbers, and $n>0$ and $0<k_{1}<k_{2}<k_{3}<\cdots$, and $n \geq \operatorname{gcd}\left(n, k_{1}\right) \geq \operatorname{gcd}\left(n, k_{1}, k_{2}\right) \geq \cdots \geq \operatorname{gcd}\left(n, k_{1}, k_{2}, \ldots\right)=1$.

Then, the problem is how to find the multiplicity and Puiseux exponents(or, Puiseux pairs) of the Puiseux expansion which is an equivalent paramtrization for the irreducible curve in (3.1.3), using the following two cases (i) and (ii), respectively :

(i) If $n$ is not greater than $k_{1}$, then the problem is easy to solve by Definition 4.1 and Theorem 4.2, which were already well-known.

(ii) If $n$ is greater than $k_{1}$, then using the inverse $t=\phi(s)$ of a conformal mapping $s=s(t)$ in (3.1.2), compute the multiplicity and Puiseux exponents of the Puiseux expansion $\left(y_{1}(s), z_{1}(s)\right)=(y(\phi(s)), z(\phi(s)))$ which is an equivalent parametrization for the curve $C$.

DEFINITION 3.2. Let $\phi(t)$ be an analytic function in a neighborhood of zero such that $\phi(0)=\phi^{\prime}(0)=\cdots=\phi^{(k)}(0)=0$, but $\phi^{(k+1)}(0) \neq 0$. Then, it is said that $\phi(t)$ has a multiplicity $k$ at $t=0$ and write $\operatorname{mult}(\phi(t), 0)=k$ for notation. Let $f(y, z)$ be in $\mathbb{C}\{y, z\}$. It is said that $f(y, z)$ has a multiplicity $\nu$ at $(y, z)=(0,0)$, denoted by $\operatorname{mul}(f(y, z),(0,0))=\nu$, if there is the least integer $\nu$ such that some partial derivative of $f$ of order $\nu$ is nonzero at the origin.

LEMMA 3.3 (THE REARRANGEMENT OF AN IRREDUCIBLE PARAMETRIZATION).

Assumption Let the curve $V$ defined by $f(y, z) \in \mathbb{C}\{y, z\}$ have an irreducible parametrization as follows:

$$
y=t^{n} \text { and } z=c_{1} t^{k_{1}}+c_{2} t^{k_{2}}+\cdots
$$

where the $c_{i}$ are nonzero complex numbers and $1 \leq n, 1 \leq k_{1}<k_{2}<\cdots$, and $n \geq \operatorname{gcd}\left(n, k_{1}\right) \geq \operatorname{gcd}\left(n, k_{1}, k_{2}\right) \geq \cdots \geq \operatorname{gcd}\left(n, k_{1}, k_{2}, \ldots\right)=1$. To get a desired rearrangement of $y=t^{n}$ and $z=\sum_{i=1}^{\infty} c_{i} t^{k_{i}}$ in the conclusion of this lemma, first we can define a finite sequence $\left\{\alpha_{1}, \alpha_{2}, \ldots, \alpha_{r+1}\right\}$ from the sequence $\left\{k_{i}: i=1,2, \ldots\right\}$ consisting of the exponents $k_{i}$ in (3.3.1) as follows:

(1) Let $\alpha_{1}=k_{1}$, and then note that $n \geq \operatorname{gcd}\left(n, \alpha_{1}\right)$. That is, either $n=$ $\operatorname{gcd}\left(n, \alpha_{1}\right)$ or $n>\operatorname{gcd}\left(n, \alpha_{1}\right)$.

(2) Let $\alpha_{2}$ be the smallest positive integer among the exponents $k_{i}$ such that $n \geq \operatorname{gcd}\left(n, \alpha_{1}\right)>\operatorname{gcd}\left(n, \alpha_{1}, k_{i}\right)$.

(3) Let $\alpha_{3}$ be the smallest positive integer among the exponents $k_{i}$ such that $n \geq \operatorname{gcd}\left(n, \alpha_{1}\right)>\operatorname{gcd}\left(n, \alpha_{1}, \alpha_{2}\right)>\operatorname{gcd}\left(n, \alpha_{1}, \alpha_{2}, k_{i}\right)$.

…...

$(\mathrm{r}+1)$ Let $\alpha_{r+1}$ be the smallest positive integer among the exponents $k_{i}$ such that $n \geq \operatorname{gcd}\left(n, \alpha_{1}\right)>\operatorname{gcd}\left(n, \alpha_{1}, \alpha_{2}\right)>\cdots>\operatorname{gcd}\left(n, \alpha_{1}, \alpha_{2}, \ldots, \alpha_{r}\right)>\operatorname{gcd}\left(n, \alpha_{1}, \alpha_{2}, \ldots\right.$, $\left.\alpha_{r}, k_{i}\right)=1$.

Let $d$ and $k$ be arbitrary positive integers. For brevity of notation, if $k$ is divisible by $d$, then we write $d \mid k$. Otherwise, we write $d \nmid k$. 
Now, let $d_{i}=\operatorname{gcd}\left(n, \alpha_{1}, \ldots, \alpha_{i}\right)$ for $1 \leq i \leq r+1$, and then $n \geq d_{1}>d_{2}>\cdots>$ $d_{r+1}$. Note that $d_{i} \mid\left(\alpha_{i}-\alpha_{1}\right), d_{i} \backslash\left(\alpha_{i+1}-\alpha_{1}\right)$, and $d_{i+1} \mid d_{i}$.

Conclusion The given irreducible parametrization of $V$ can be rearranged in $t$ as follows:

$$
\begin{aligned}
y= & t^{n} \\
z= & c_{1} t^{\alpha_{1}}\left\{\left(1+c_{11} t^{d_{1}}+c_{12} t^{2 d_{1}}+\cdots+c_{1 p_{1}} t^{p_{1} d_{1}}\right)\right. \\
& +t^{\alpha_{2}-\alpha_{1}}\left(c_{20}+c_{21} t^{d_{2}}+c_{22} t^{2 d_{2}}+\cdots+c_{2 p_{2}} t^{p_{2} d_{2}}\right) \\
& +\ldots \ldots \\
& +t^{\alpha_{r}-\alpha_{1}}\left(c_{r 0}+c_{r 1} t^{d_{r}}+c_{r 2} t^{2 d_{r}}+\cdots+c_{r p_{r}} t^{p_{r} d_{r}}\right) \\
& \left.+t^{\alpha_{r+1}-\alpha_{1}}\left(c_{r+1,0}+\sum_{k+1}^{\infty} c_{r+1, k} t^{k}\right)\right\}
\end{aligned}
$$

satisfying the properties (i), (ii) and (iii).

(i) $c_{10}=1, c_{20}, c_{30}, \ldots, c_{r+1,0}$ are all nonzero complex numbers.

(ii) $p_{1}, p_{2}, \ldots, p_{r}$ are nonnegative integers such that

$$
\begin{gathered}
\alpha_{1}+p_{1} d_{1}<\alpha_{2}<\alpha_{1}+\left(p_{1}+1\right) d_{1} \\
\alpha_{2}+p_{2} d_{2}<\alpha_{3}<\alpha_{2}+\left(p_{2}+1\right) d_{2} \\
\ldots \ldots \\
\alpha_{r-1}+p_{r-1} d_{r-1}<\alpha_{r}<\alpha_{r-1}+\left(p_{r-1}+1\right) d_{r-1} \\
\alpha_{r}+p_{r} d_{r}<\alpha_{r+1}<\alpha_{r}+\left(p_{r}+1\right) d_{r}
\end{gathered}
$$

(iii) Let $S$ be the set which consists of the remaining coefficients in $t$, that is,

$$
\begin{aligned}
S= & \left\{c_{11}, c_{12}, \ldots, c_{1, p_{1}}\right\} \cup\left\{c_{21}, c_{22}, \ldots, c_{2, p_{2}}\right\} \cup \ldots \\
& \cup\left\{c_{r 1}, c_{r 2}, \ldots, c_{r, p_{r}}\right\} \cup\left\{c_{r+1, k}: k=1,2, \ldots\right\} .
\end{aligned}
$$

Then, any element of $S$ is either zero or nonzero.

Note that $p_{i}$ may be zero for some $i, 1 \leq i \leq r$. In particular, if $p_{i}=0$ for $1 \leq i \leq r$, then note that $c_{i 1}, c_{i 2}, \ldots, c_{i, p_{i}}$ are all zero except for $c_{i o}$.

Proof of Lemma 3.3. It is trivial.

TheOREM 3.4 (AN EQUiVALENCE OF IRREDUCIBLE PARAMETRIZATION).

Assumption We may assume without loss of generality that the curve $V$ defined by $\overline{f(y, z) \in \mathbb{C}\{y, z\}}$ at the origin has an irreducible parametrization as follows:

$$
\begin{aligned}
y= & t^{n}, \\
z= & c t^{\alpha_{1}}\left\{\left(1+D_{1}(t)\right)+t^{\alpha_{2}-\alpha_{1}}\left(c_{20}+D_{2}(t)\right)+\cdots\right. \\
& \left.+t^{\alpha_{r}-\alpha_{1}}\left(c_{r 0}+D_{r}(t)\right)+t^{\alpha_{r+1}-\alpha_{1}}\left(c_{r+1,0}+D_{r+1}(t)\right)\right\} \\
= & c t^{\alpha_{1}}(1+H(t)) \text { or } \\
y= & t^{n}, \\
z= & c t^{\alpha_{1}}\left\{1+D_{1}(t)\right\}+c t^{\alpha_{2}}\left\{c_{20}+D_{2}(t)\right\}+\cdots \\
& +c t^{\alpha_{r}}\left\{c_{r 0}+D_{r}(t)\right\}+c t^{\alpha_{r+1}}\left\{c_{r+1,0}+D_{r+1}(t)\right\}
\end{aligned}
$$


where

(i) $1 \leq n$ and $1 \leq \alpha_{1}<\alpha_{2}<\cdots<\alpha_{r+1}$,

(ii) $n \geq d_{1}>d_{2}>\cdots>d_{r+1}=1$ with $\operatorname{gcd}\left(n, \alpha_{1}, \alpha_{2}, \ldots, \alpha_{i}\right)=d_{i}$ for $1 \leq i \leq$ $r+1$,

(iii) $p_{1}, p_{2}, \ldots, p_{r}$ are nonnegative integers such that

$$
\begin{gathered}
\alpha_{1}+p_{1} d_{1}<\alpha_{2}<\alpha_{1}+\left(p_{1}+1\right) d_{1}, \\
\alpha_{2}+p_{2} d_{2}<\alpha_{3}<\alpha_{2}+\left(p_{2}+1\right) d_{2}, \\
\quad \ldots \ldots \\
\alpha_{r-1}+p_{r-1} d_{r-1}<\alpha_{r}<\alpha_{r-1}+\left(p_{r-1}+1\right) d_{r-1} \\
\alpha_{r}+p_{r} d_{r}<\alpha_{r+1}<\alpha_{r}+\left(p_{r}+1\right) d_{r}
\end{gathered}
$$

(iv) let

$$
\begin{aligned}
D_{1}(t)= & \sum_{i=1}^{p_{1}} c_{1 i} t^{i d_{1}} \in \mathbb{C}[t], \\
D_{2}(t)= & \sum_{i=1}^{p_{2}} c_{2 i} t^{i d_{2}} \in \mathbb{C}[t], \\
\cdots & \ldots \\
D_{r}(t)= & \sum_{i=1}^{p_{r}} c_{r i} t^{i d_{r}} \in \mathbb{C}[t], \\
D_{r+1}(t)= & \sum_{k=1}^{\infty} c_{r+1, k} t^{k} \in \mathbb{C}\{t\}, \\
1+H(t)= & 1+D_{1}(t)+t^{\alpha_{2}-\alpha_{1}}\left(c_{20}+D_{2}(t)\right)+\cdots \\
& +t^{\alpha_{r}-\alpha_{1}}\left(c_{r 0}+D_{r}(t)\right)+t^{\alpha_{r+1}-\alpha_{1}}\left(c_{r+1,0}+D_{r+1}(t)\right),
\end{aligned}
$$

(v) $c, c_{10}=1, c_{20}, c_{30}, \ldots, c_{r+1,0}$ are all nonzero complex numbers.

Conclusion We have the followings: Observe that (I) of two statements (I) and (II) below may be omitted, in order to simplify the statements for Conclusion, if necessary.

(I) In preparation for the construction of an equivalent irreducible parametrization of $V$, let $s$ be the new parameter defined by

$$
s(t)=c^{\frac{1}{\alpha_{1}}} t(1+H(t))^{\frac{1}{\alpha_{1}}}
$$

where

(i) $c^{\frac{1}{\alpha_{1}}}$ is a complex root such that $\omega^{\alpha_{1}}=c$,

(ii) $s=s(t)$ is a conformal mapping of $t$ at the origin,

(iii) $z=s^{\alpha_{1}}$.

Then $t=c^{-\frac{1}{\alpha_{1}}} s(1+H(t))^{-\frac{1}{\alpha_{1}}}$, as $t=\phi(s) \in \mathbb{C}\{s\}$, can be written as follows: 
Note that $y=(\phi(s))^{n}$.

$$
\begin{aligned}
t= & \phi(s) \\
= & c^{-\frac{1}{\alpha_{1}}} s\left\{1+Q_{1}(s)+s^{\alpha_{2}-\alpha_{1}}\left(B_{20}+Q_{2}(s)\right)\right. \\
& \left.+\cdots+s^{\alpha_{r}-\alpha_{1}}\left(B_{r 0}+Q_{r}(s)\right)+s^{\alpha_{r+1}-\alpha_{1}}\left(B_{r+1,0}+Q_{r+1}(s)\right)\right\}
\end{aligned}
$$

where

$$
\begin{aligned}
& B_{20}= \frac{c_{20}}{-\alpha_{1}}\left(c^{-\frac{1}{\alpha_{1}}}\right)^{\alpha_{2}-\alpha_{1}}, B_{30}=\frac{c_{30}}{-\alpha_{1}}\left(c^{-\frac{1}{\alpha_{1}}}\right)^{\alpha_{3}-\alpha_{1}}, \ldots, \\
& B_{r+1,0}=\frac{c_{r+1,0}}{-\alpha_{1}}\left(c^{-\frac{1}{\alpha_{1}}}\right)^{\alpha_{r+1}-\alpha_{1}}, \\
& Q_{1}(s)= B_{11} s^{d_{1}}+B_{12} s^{2 d_{1}}+\cdots+B_{1, p_{1}} s^{p_{1} d_{1}} \in \mathbb{C}[s], \\
& Q_{2}(s)= B_{21} s^{d_{2}}+B_{22} s^{2 d_{2}}+\cdots+B_{2, p_{2}} s^{p_{2} d_{2}} \in \mathbb{C}[s], \\
& \cdots \cdots \\
& Q_{r}(s)= B_{r 1} s^{d_{r}}+B_{r 2} s^{2 d_{r}}+\cdots+B_{r, p_{r}} s^{p_{r} d_{r}} \in \mathbb{C}[s], \\
& Q_{r+1}(s)=\sum_{k=1}^{\infty} B_{r+1, k} s^{k} \in \mathbb{C}\{s\}
\end{aligned}
$$

such that all the $B_{i j}$ are complex numbers and that in particular the $B_{i 0}$ are nonzero for $2 \leq i \leq r+1$. Note that $Q_{i}(0)=0$ for $1 \leq i \leq r+1$.

(II) The equivalent parametrization with the new parameter $s$ for $V$ can be analytically written in the following form:

$$
\begin{aligned}
z= & s^{\alpha_{1}} \\
y= & c^{-\frac{n}{\alpha_{1}}} s^{n}\left\{1+Q_{1}^{*}(s)+s^{\alpha_{2}-\alpha_{1}}\left(b_{20}+Q_{2}^{*}(s)\right)\right. \\
& \left.+s^{\alpha_{3}-\alpha_{1}}\left(b_{30}+Q_{3}^{*}(s)\right)+\cdots+s^{\alpha_{r+1}-\alpha_{1}}\left(b_{r+1,0}+Q_{r+1}^{*}(s)\right)\right\}
\end{aligned}
$$

where

$$
\begin{aligned}
b_{20}= & \frac{n}{-\alpha_{1}} c_{20} c^{\frac{1}{-\alpha_{1}}\left(\alpha_{2}-\alpha_{1}\right)}, b_{30}=\frac{n}{-\alpha_{1}} c_{30} c^{\frac{1}{-\alpha_{1}}\left(\alpha_{3}-\alpha_{1}\right)}, \ldots, \\
& b_{r+1,0}=\frac{n}{-\alpha_{1}} c_{r+1,0} c^{\frac{1}{-\alpha_{1}}\left(\alpha_{r+1}-\alpha_{1}\right)}, \\
Q_{1}^{*}(s)= & b_{11} s^{d_{1}}+b_{12} s^{2 d_{1}}+\cdots+b_{1, p_{1}} s^{p_{1} d_{1}} \in \mathbb{C}[s], \\
Q_{2}^{*}(s)= & b_{21} s^{d_{2}}+b_{22} s^{2 d_{2}}+\cdots+b_{2, p_{2}} s^{p_{2} d_{2}} \in \mathbb{C}[s], \\
& \cdots \cdots \\
Q_{r}^{*}(s)= & b_{r 1} s^{d_{r}}+b_{r 2} s^{2 d_{r}}+\cdots+b_{r, p_{r}} s^{p_{r} d_{r}} \in \mathbb{C}[s], \\
Q_{r+1}^{*}(s)= & \sum_{k=1}^{\infty} b_{r+1, k} s^{k} \in \mathbb{C}\{s\}
\end{aligned}
$$

such that all the $b_{i j}$ are complex numbers and that in particular the $b_{i 0}$ are nonzero for $2 \leq i \leq r+1$. Note that $Q_{i}^{*}(0)=0$ for all $i=2,3, \ldots, r+1$.

Remark: Observe by (3.4.5) and (3.4.7) that 


$$
b_{20}=n B_{20}, b_{30}=n B_{30}, \ldots, b_{r+1,0}=n B_{r+1,0} .
$$

Proof of Theorem 3.4. First, let us find a new a parameter $s$ for $V$ of either (3.4.3) or (3.4.6) such that $z(t)=s^{\alpha_{1}}$ with $\alpha_{1}=k_{1}$. Because $z(t)=c t^{\alpha_{1}}(1+H(t))$ by (3.4.1), where $H(t)$ is an analytic function in $\mathbb{C}\{t\}$ with $H(0)=0$, then $s=\psi(t)$ can be written in the form

$$
s=c^{\frac{1}{\alpha_{1}}} t(1+H(t))^{\frac{1}{\alpha_{1}}}
$$

where

(i) $c^{\frac{1}{\alpha_{1}}}$ is a complex root such that $\omega^{\alpha_{1}}=c$,

(ii) $H(t)$ is an analytic function defined by (3.4.2).

Since $H(t)$ is analytic at the origin with $H(0)=0$, then $\psi(t)$ is analytic at the origin such that $\psi(0)=0$ and $\psi^{\prime}(0) \neq 0$. That is, $\psi(t)$ is a conformal mapping at the origin. So at $t=0, s=\psi(t)$ has an inverse analytic function, denoted by $t=\phi(s)$ with $\phi(0)=0$. Therefore, by (3.4.8) we have

$$
\begin{aligned}
t & =c^{-\frac{1}{\alpha_{1}}} s(1+H(t))^{-\frac{1}{\alpha_{1}}} \quad \text { with } \quad H(0)=0 \\
& =c^{-\frac{1}{\alpha_{1}}} s\left(1+H(\phi(s))^{-\frac{1}{\alpha_{1}}} \text { with } \quad H(\phi(0))=0 .\right.
\end{aligned}
$$

Note by $y=t^{n}$ that

$$
\begin{aligned}
z & =s^{\alpha_{1}} \quad \text { and also } \\
y & =c^{-\frac{n}{\alpha_{1}}} s^{n}(1+H(t))^{-\frac{n}{\alpha_{1}}} \\
& =c^{-\frac{n}{\alpha_{1}}} s^{n}(1+H(\phi(s)))^{-\frac{n}{\alpha_{1}}} .
\end{aligned}
$$

So, for the proof, it is enough to consider two cases, respectively: (i) $\operatorname{gcd}\left(n_{1}, \alpha_{1}\right)=1$ and (ii) $\operatorname{gcd}\left(n_{1}, \alpha_{1}\right)>1$.

In preparation for the proof, write $H_{1}(s)=H(\phi(s))$ for brevity. Then $H_{1}(s)$ is an analytic function of $s$ with $H_{1}(0)=0$, and so we will find a convergent power series of $\left(1+H_{1}(s)\right)^{-\frac{n}{\alpha_{1}}}$ at $s=0$. Note by the binomial series expansion that for $\lambda=\frac{q}{p}$ with some nonzero integers $p$ and $q$

$$
\begin{aligned}
\left(1+H_{1}(s)\right)^{\lambda}= & +\left(\begin{array}{l}
\lambda \\
1
\end{array}\right) H_{1}(s)+\left(\begin{array}{l}
\lambda \\
2
\end{array}\right)\left(H_{1}(s)\right)^{2}+\cdots \\
& +\left(\begin{array}{l}
\lambda \\
k
\end{array}\right)\left(H_{1}(s)\right)^{k}+\cdots
\end{aligned}
$$

where the binomial coefficients are defined by

$$
\left(\begin{array}{l}
\lambda \\
k
\end{array}\right)=\frac{\lambda(\lambda-1) \cdots(\lambda-k+1)}{1 \cdot 2 \cdots k}
$$

Then, it is trivial to prove that

$$
\left(1+H_{1}(s)\right)^{\lambda}=1+u(s)
$$

where $u(s)$ is an analytic function of $s$ at 0 with $u(0)=0$ because $H_{1}(0)=0$. Now, by $(3.4 .9),(3.4 .10)$ and $(3.4 .13)$, it is clear that

$$
\begin{aligned}
& t=c^{-\frac{1}{\alpha_{1}}} s\left(1+u_{1}(s)\right) \text { and } z=s^{\alpha_{1}} \\
& y=c^{-\frac{n}{\alpha_{1}}} s^{n}\left(1+u_{2}(s)\right)
\end{aligned}
$$


where $u_{1}(s)$ and $u_{2}(s)$ are analytic functions of $s$ at the origin with $u_{1}(s)=u_{2}(0)=0$. Now, consider two cases mentioned above.

The first case: Let $\operatorname{gcd}\left(n, \alpha_{1}\right)=1$. Then, the proof of this case is done by (3.4.14).

The second case: Let $\operatorname{gcd}\left(n, \alpha_{1}\right)>1$. To prove this theorem, it is enough to consider this case only. For the proof, by (3.4.2), (3.4.8) and (3.4.9), $t=\phi(s)$ can be written as follows:

$$
t=c^{-\frac{1}{\alpha_{1}}} s(1+H(t))^{-\frac{1}{\alpha_{1}}}
$$

where

$$
\begin{aligned}
D_{1}(t)= & \sum_{i=1}^{p_{1}} c_{1 i} t^{i d_{1}} \in \mathbb{C}[t] \\
D_{2}(t)= & \sum_{i=1}^{p_{2}} c_{2 i} t^{i d_{2}} \in \mathbb{C}[t] \\
\ldots & \\
D_{r}(t)= & \sum_{i=1}^{p_{r}} c_{r i} t^{i d_{r}} \in \mathbb{C}[t] \\
D_{r+1}(t)= & \sum_{k=1}^{\infty} c_{r+1, k} t^{k} \in \mathbb{C}\{t\} \\
H(t)= & D_{1}(t)+t^{\alpha_{2}-\alpha_{1}}\left(c_{20}+D_{2}(t)\right)+\cdots \\
& +t^{\alpha_{r}-\alpha_{1}}\left(c_{r 0}+D_{r}(t)\right)+t^{\alpha_{r+1}-\alpha_{1}}\left(c_{r+1,0}+D_{r+1}(t)\right) .
\end{aligned}
$$

In preparation for the computation of an analytic function $t=\phi(s)$, first we will prove the following lemma.

LEMMA 3.4.1. Let $H(t)$ be given by (3.4.16). Then we get the followings:

$$
\begin{aligned}
(1+H(t))^{-\frac{1}{\alpha_{1}}}= & 1+D_{1}^{*}(t)+t^{\alpha_{2}-\alpha_{1}}\left(-\frac{c_{20}}{\alpha_{1}}+D_{2}^{*}(t)\right) \\
& +t^{\alpha_{3}-\alpha_{1}}\left(-\frac{c_{30}}{\alpha_{1}}+D_{3}^{*}(t)\right)+\cdots+t^{\alpha_{r}-\alpha_{1}}\left(-\frac{c_{r 0}}{\alpha_{1}}+D_{r}^{*}(t)\right) \\
& +t^{\alpha_{r+1}-\alpha_{1}}\left(-\frac{c_{r+1,0}}{\alpha_{1}}+D_{r+1}^{*}(t)\right)
\end{aligned}
$$

where

$$
\begin{aligned}
& D_{1}^{*}(t)=A_{11} t^{d_{1}}+A_{12} t^{2 d_{1}}+\cdots+A_{1, p_{1}} t^{p_{1} d_{1}} \in \mathbb{C}[t] \\
& D_{2}^{*}(t)=A_{21} t^{d_{2}}+A_{22} t^{2 d_{2}}+\cdots+A_{2, p_{2}} t^{p_{2} d_{2}} \in \mathbb{C}[t] \\
& \quad \cdots \cdots \\
& D_{r}^{*}(t)=A_{r 1} t^{d_{r}}+A_{r_{2}} t_{2 d_{r}}+\cdots+A_{r, p_{r}} t^{p_{r} d_{r}} \in \mathbb{C}[t] \\
& D_{r+1}^{*}(t)=\sum_{k=1}^{\infty} A_{r+1, k} t^{k} \in \mathbb{C}\{t\}
\end{aligned}
$$


such that all the $A_{i j}$ are complex numbers.

In general, for any $\lambda=\frac{q_{1}}{q_{2}}$ with nonzero integers $q_{1}$ and $q_{2},(1+H(t))^{\lambda}$ can be rewritten as follows: We use the same notations and meanings as in (3.4.17).

$$
\begin{aligned}
(1+H(t))^{\lambda}=1 & +D_{1}^{*}(t)+t^{\alpha_{2}-\alpha_{1}}\left(\lambda c_{20}+D_{2}^{*}(t)\right) \\
& +t^{\alpha_{3}-\alpha_{2}}\left(\lambda c_{30}+D_{3}^{*}(t)\right)+\cdots+t^{\alpha_{r}-\alpha_{1}}\left(\lambda c_{r 0}+D_{r}^{*}(t)\right) \\
& +t^{\alpha_{r+1}-\alpha_{1}}\left(\lambda c_{r+1,0}+D_{r+1}^{*}(t)\right)
\end{aligned}
$$

Proof of Lemma 3.4.1. To prove the lemma, we use some notations and observations as follows:

(i) Let $d$ and $k$ be given positive integers. If $k$ is divisible by $d$, then we write $d \mid k$. Otherwise, we write $d \backslash k$.

(ii) Let $g(t)$ be in $\mathbb{C}\{t\}$. If $t^{k}$ is appeared with a nonzero coefficient in $g(t)$, then we say that $t^{k}$ belongs to $g(t)$ or $t^{k} \in g(t)$. Otherwise, write $t^{k} \notin g(t)$.

(iii) Let $d_{i}=\operatorname{gcd}\left(n, \alpha_{1}, \ldots, \alpha_{i}\right)$ for $1 \leq i \leq r+1$, and then $n \geq d_{1}>d_{2}>\cdots>$ $d_{r+1}=1$. Note that $d_{i} \mid\left(\alpha_{i}-\alpha_{1}\right) ; d_{i} \backslash\left(\alpha_{i+1}-\alpha_{1}\right)$, and $d_{i+1} \mid d_{i}$ for $i=1,2, \ldots, r$.

(iv) Whenever $t^{k}$ belongs to $D_{i}(t)$ of (3.4.16) for each $i=1,2, \ldots, r+1$, then $d_{i} \mid k$. If $t^{k} \in D_{i}(t) D_{j}(t)$ with $1 \leq i<j \leq r+1$, then $d_{j} \mid k$.

(v) For each $i=1,2, \ldots, r+1$, the coefficient $c_{i 0}$ of $t^{\alpha_{i}-\alpha_{1}}$ of $H(t)$ in $\mathbb{C}\{t\}$ is nonzero as we have seen in Lemma 3.3.

$(\mathrm{vi})^{*} \quad \alpha_{i+1}-\alpha_{1}$ is the smallest positive integer among all $\mu$ such that $t^{\mu} \in H(t)$ and $\mu$ is divisible by $d_{i+1}$, but cannot be divisible by $d_{i}$.

Now, as we have seen in (3.4.11), note by the binomial series expansion that

$$
\begin{aligned}
(1+H(t))^{\lambda}= & +\left(\begin{array}{l}
\lambda \\
1
\end{array}\right) H(t)+\left(\begin{array}{l}
\lambda \\
2
\end{array}\right) H^{2}(t) \\
& +\left(\begin{array}{l}
\lambda \\
3
\end{array}\right) H^{3}(t)+\cdots
\end{aligned}
$$

where the binomial coefficients are defined by

$$
\left(\begin{array}{l}
\lambda \\
k
\end{array}\right)=\frac{\lambda(\lambda-1) \cdots(\lambda-k+1)}{1 \cdot 2 \cdots k} .
$$

By (3.4.15) and (3.4.16) with the above observations (vi)*, we have another observation (vii)*:

(vii)* Whenever $\nu$ is an arbitrary positive integer such that $t^{\nu} \in H^{j}(t)$ for any $j \geq 2$ and that $\nu$ is divisible by $d_{i+1}$, but cannot be divisible by $d_{i}$, then $\nu>\alpha_{i+1}-\alpha_{1}$ by (vi)* because $H(0)=0$ and $j \geq 2$.

Thus, the proof of the lemma is done by (3.4.19), (vi)* and (vii)*.

For brevity of notations of (3.4.17) and (3.4.18), let

$$
\begin{aligned}
E_{1}^{*}(t) & =D_{1}^{*}(t) \\
E_{2}^{*}(t) & =t^{\alpha_{2}-\alpha_{1}}\left(\lambda c_{20}+D_{2}^{*}(t)\right) \\
\cdots & \cdots \\
E_{r}^{*}(t) & =t^{\alpha_{r}-\alpha_{1}}\left(\lambda c_{r 0}+D_{r}^{*}(t)\right) \\
E_{r+1}^{*}(t) & =t^{\alpha_{r+1}-\alpha_{1}}\left(\lambda c_{r+1,0}+D_{r+1}^{*}(t)\right) \\
H^{*}(t) & =E_{1}^{*}(t)+E_{2}^{*}(t)+\cdots+E_{r+1}^{*}(t)
\end{aligned}
$$


Then, $(1+H(t))^{\lambda}$ of $(4.22)$ can be rewritten in the form

$$
\begin{aligned}
(1+H(t))^{\lambda} & =1+H^{*}(t) \\
& =1+E_{1}^{*}(t)+E_{2}^{*}(t)+\cdots+E_{r+1}^{*}(t) .
\end{aligned}
$$

Now, in order to prove (I), replacing $\lambda$ in (3.4.20) and (3.4.21) by $\frac{1}{-\alpha_{1}}$, it is enough to show each step inductively in the following lemma.

$\left.{ }^{* *}\right)$ Before the next lemma is proved, first of all, note that the equation in (3.4.30) of the $r$-th step in the next lemma is the same as the equation in (3.4.4) of (I) in the conclusion of this theorem.

Let us prove the lemma mentioned just above.

LEMMA 3.4.2. As in the conclusion (I) of the theorem, we may begin with the given finite sequence $\left\{\alpha_{k}: k=1,2, \ldots, r+1\right.$ with $\left.r \geq 1\right\}$. Then each of the following steps can be described by induction on the integer $l \leq r+1$ as follows: Let $t=\phi(s)$.

Step 1

(i) $s^{\alpha_{2}-\alpha_{1}} \in E_{2}^{*}(\phi(s))$, but $s^{\alpha_{2}-\alpha_{1}} \notin E_{i}^{*}(\phi(s))$ for all $i \neq 2$.

(ii) $s^{\alpha_{2}-\alpha_{1}} \in H^{*}(\phi(s))$ with coefficient $\frac{c_{20}}{-\alpha_{1}}\left(c^{-\frac{1}{\alpha_{1}}}\right)^{\alpha_{2}-\alpha_{1}}$.

(iii) Then $\phi(s)$ can be defined by $\phi_{1}(s)$,

$$
\begin{aligned}
\phi_{1}(s)= & c^{-\frac{1}{\alpha_{1}}} s\left\{1+Q_{1}(s)+B_{20} s^{\alpha_{2}-\alpha_{1}}\right. \\
& \left.+\sum_{k>\alpha_{2}-\alpha_{1}} a_{k} s_{k}\right\}
\end{aligned}
$$

where

$$
\begin{aligned}
B_{20} & =-\frac{c_{20}}{\alpha_{1}}\left(c^{-\frac{1}{\alpha_{1}}}\right)^{\alpha_{2}-\alpha_{1}}, \\
Q_{1}(s) & =B_{11} s^{d_{1}}+B_{12} s^{2 d_{1}}+\cdots+B_{1, p_{1}} s^{p_{1} d_{1}}
\end{aligned}
$$

such that the $B_{1 j}$ are the coefficients, some of which may be zero. Note that if $p_{1}=0$, then $Q_{1}(s)$ is identically zero.

Step 2

(i) $s^{\alpha_{3}-\alpha_{1}} \in E_{3}^{*}(\phi(s))$, but $s^{\alpha_{3}-\alpha_{1}} \notin E_{i}^{*}(\phi(s))$ for all $i \neq 3$.

(ii) $s^{\alpha_{3}-\alpha_{1}} \in H^{*}(\phi(s))$ with coefficient $-\frac{c_{30}}{\alpha_{1}}\left(c^{-\frac{1}{\alpha_{1}}}\right)^{\alpha_{3}-\alpha_{1}}$.

(iii) Then $\phi(s)$ can be defined by $\phi_{2}(s)$,

$$
\begin{gathered}
\phi_{2}(s)=c^{-\frac{1}{\alpha_{1}}} s\left\{1+Q_{1}(s)+s^{\alpha_{2}-\alpha_{1}}\left(B_{20}+Q_{2}(s)\right)\right. \\
\left.\cdot+B_{30} s^{\alpha_{3}-\alpha_{1}}+\sum_{k>\alpha_{3}-\alpha_{1}} a_{k} s_{k}\right\}
\end{gathered}
$$

where

$$
\begin{aligned}
B_{20} & =-\frac{c_{20}}{\alpha_{1}}\left(c^{-\frac{1}{\alpha_{1}}}\right)^{\alpha_{2}-\alpha_{1}} \\
B_{30} & =-\frac{c_{30}}{\alpha_{1}}\left(c^{-\frac{1}{\alpha_{1}}}\right)^{\alpha_{3}-\alpha_{1}} \\
Q_{2}(s) & =B_{21} s^{d_{2}}+B_{22} s^{2 d_{2}}+\cdots+B_{2, p_{2}} s^{p_{2} d_{2}}
\end{aligned}
$$


such that the $B_{2 j}$ are the coefficients, some of which may be zero. Note that if $p_{2}=0$, then $Q_{2}(s)$ is identically zero.

\section{Step (r-1)}

(i) $s^{\alpha_{r}-\alpha_{1}} \in E_{r}^{*}(\phi(s))$, but $s^{\alpha_{r}-\alpha_{1}} \notin E_{i}^{*}(\phi(s))$ for all $i \neq r$.

(ii) $s^{\alpha_{r}-\alpha_{1}} \in H^{*}(\phi(s))$ with coefficient $-\frac{c_{r 0}}{\alpha_{1}}\left(c^{-\frac{1}{\alpha_{1}}}\right)^{\alpha_{r}-\alpha_{1}}$.

(iii) Then $\phi(s)$ can be defined by $\phi_{r-1}(s)$,

$$
\begin{aligned}
\phi_{r-1}(s)= & c^{-\frac{1}{\alpha_{1}}} s\left\{1+Q_{1}(s)+s^{\alpha_{2}-\alpha_{1}}\left(B_{20}+Q_{2}(s)\right)+\cdots\right. \\
& \left.+s^{\alpha_{r-1}-\alpha_{1}}\left(B_{r-1,0}+Q_{r-1}(s)\right)+B_{r 0} s^{\alpha_{r}-\alpha_{1}}+\sum_{k>\alpha_{r}-\alpha_{1}} a_{k} s^{k}\right\}
\end{aligned}
$$

where

$$
\begin{aligned}
B_{20} & =-\frac{c_{20}}{\alpha_{1}}\left(c^{-\frac{1}{\alpha_{1}}}\right)^{\alpha_{r}-\alpha_{1}} \\
& \ldots \cdots \\
B_{r 0} & =-\frac{c_{r o}}{\alpha_{1}}\left(c^{-\frac{1}{\alpha_{1}}}\right)^{\alpha_{r}-\alpha_{1}} \\
Q_{r-1}(s) & =B_{r-1,1} s^{d_{r-1}}+B_{r-1,2} s^{2 d_{r-1}}+\cdots+B_{r-1, r-1} s^{p_{r-1} d_{r-1}}
\end{aligned}
$$

such that the $B_{r-1, j}$ are the coefficients, some of which may be zero. Note that if $p_{r-1}=0$, then $Q_{r-1}(s)$ is identically zero.

Step $\mathrm{r}$

(i) $s^{\alpha_{r+1}-\alpha_{1}} \in E_{r+1}^{*}(\phi(s))$, but $s^{\alpha_{r+1}-\alpha_{1}} \notin E_{i}^{*}(\phi(s))$ for all $i \neq r+1$.

(ii) $s^{\alpha_{r+1}-\alpha_{1}} \in H^{*}(\phi(s))$ with coefficient $-\frac{c_{r+1,0}}{\alpha_{1}}\left(c^{-\frac{1}{\alpha_{1}}}\right)^{\alpha_{r+1}-\alpha_{1}}$.

(iii) Then $\phi(s)$ can be defined by $\phi_{r}(s)$,

$$
\begin{aligned}
\phi_{r}(s)= & c^{-\frac{1}{\alpha_{1}}} s\left\{1+Q_{1}(s)+s^{\alpha_{2}-\alpha_{1}}\left(B_{20}+Q_{2}(s)+\cdots\right.\right. \\
& \left.+s^{\alpha_{r}-\alpha_{1}}\left(B_{r 0}+Q_{r}(s)\right)+B_{r+1,0} s^{\alpha_{r+1}-\alpha_{1}}+\sum_{k>\alpha_{r+1}-\alpha_{1}} a_{k} s^{k}\right\}
\end{aligned}
$$

where

$$
\begin{aligned}
B_{20} & =-\frac{c_{20}}{\alpha_{1}}\left(c^{-\frac{1}{\alpha_{1}}}\right)^{\alpha_{2}-\alpha_{1}} \\
& \ldots \ldots \\
B_{r+1,0} & =-\frac{c_{r+1,0}}{\alpha_{1}}\left(c^{-\frac{1}{\alpha_{1}}}\right)^{\alpha_{r+1}-\alpha_{1}} \\
Q_{r}(s) & =B_{r 1} s^{d_{r}}+B_{r 2} s^{2 d_{r}}+\cdots+B_{r r} s^{p_{r} d_{r}}
\end{aligned}
$$

such that the $B_{r j}$ are the coefficients, some of which may be zero. Note that if $p_{r}=0$, then $Q_{r}(s)$ is identically zero. (II).

Proof of Lemma 3.4.2. The proof will follow from two cases Case (I) and Case 
Case (I): First, we will prove Step 1 of Lemma 3.4.2.

Case (II): For the induction proof of Lemma 3.4.2, after the proof of Step 1 by Case (I), suppose by the induction assumption on the integer $j$ with $1 \leq j \leq r$ that Step $j$ with $1 \leq j \leq(r-1)$ is true. Then, in this case we will prove that Step $(j+1)$ is true.

Instead of the direct proofs for Case (I) and Case (II), first we will construct two easy and elementary sublemmas for the statements which are needed to prove both Case (I) and Case (II), and next we will show these sublemmas, which gives the proofs of Case (I) and Case (II), respectively in the following way:

(A) For the proof of Case (I), we will construct Sublemma I, which is equivalent to the statement of Step 1.

(B) Next, for the proof of Case (II), we will construct Sublemma II, which is equivalent to the statement for the proof of Case (II).

In preparation for the proof of Case (I), it is very clear that the following sublemma by using the induction on the integer $p_{1}$ is just the restatement of Step 1. Let us write down it. Then we will prove it, just after the construction of another sublemma, that is, Sublemma II.

Sublemma I. Let $\phi(s)=c^{-\frac{1}{\alpha_{1}}} s\left(1+\sum_{k>0}^{\infty} a_{k} s^{k}\right)$. Note that $p_{1}$ is the nonnegative integer such that $p_{1} d_{1}<\alpha_{2}-\alpha_{1}<\left(p_{1}+1\right) d_{1}$. Then there are two subcases: $p_{1}=0$ and $p_{1}>0$.

Subcase (I-1) Let $p_{1}=0$.

(i) $s^{\alpha_{2}-\alpha_{1}} \in E_{2}^{*}(\phi(s))$, but $s^{\alpha_{2}-\alpha_{1}} \notin E_{i}^{*}(\phi(s))$ for all $i \neq 2$.

(ii) $s^{\alpha_{2}-\alpha_{1}} \in H^{*}(\phi(s))$ with coefficient $-\frac{1}{\alpha_{1}} c_{20}\left(c^{-\frac{1}{\alpha_{1}}}\right)^{\alpha_{2}-\alpha_{1}}$.

(iii) Then $\phi(s)$ can be defined by $\phi_{1}(s)$,

$$
\begin{gathered}
\phi_{1}(s)=c^{-\frac{1}{\alpha_{1}}} s\left\{1+Q_{1}(s)+B_{20} s^{\alpha_{2}-\alpha_{1}}+\sum_{k>\alpha_{2}-\alpha_{1}} a_{k} s^{k}\right\} \\
\text { with } B_{20}=-\frac{1}{\alpha_{1}} c_{20}\left(c^{-\frac{1}{\alpha_{1}}}\right)^{\alpha_{2}-\alpha_{1}}
\end{gathered}
$$

where $Q_{1}(s)$ is identically zero.

Subcase (I-2) Let $p_{1}>0$. In this case, instead of proving Step 1 directly, first show that the statement (a) is true. After then, we will prove that the next statement $(b)$ is true. Note that the statement (b) and the statement of Step 1 are the same.

(a) For each integer $\tau=0,1,2, \ldots, p_{1}, \phi(s)$ can be defined by $\phi_{1, \tau}(s)$,

$$
\phi_{1, \tau}(s)=c^{-\frac{1}{\alpha_{1}}} s\left\{1+Q_{1, \tau}^{*}(s)+\sum_{k>\tau d_{1}} a_{k} s^{k}\right\}
$$

where

$$
Q_{1, \tau}^{*}(s)=B_{11}^{*} s^{d_{1}}+B_{12}^{*} s^{2 d_{1}}+\cdots+B_{1 \tau}^{*} s^{\tau d_{1}}
$$

such that the $B_{1 i}^{*}$ are complex numbers for $1 \leq i \leq \tau$. If $\tau=0$, note that $Q_{1, \tau}^{*}(s)$ is zero.

(b) Now, if $\tau=p_{1}$, then we may begin with $\phi(s)=\phi_{1, p_{1}}(s)$ in $(a)$. The remaining part of Step 1 just follows from

(i) $s^{\alpha_{2}-\alpha_{1}} \in E_{2}^{*}(\phi(s))$, but $s^{\alpha_{2}-\alpha_{1}} \notin E_{i}^{*}(\phi(s))$ for all $i \neq 2$. 
(ii) $s^{\alpha_{2}-\alpha_{1}} \in H^{*}(\phi(s))$ with coefficient $-\frac{c_{20}}{\alpha_{1}}\left(c^{-\frac{1}{\alpha_{1}}}\right)^{\alpha_{2}-\alpha_{1}}$.

(iii) Then $\phi(s)$ can be defined by $\phi_{1}(s)$,

$$
\begin{aligned}
\phi_{1}(s)= & c^{-\frac{1}{\alpha_{1}}} s\left\{1+Q_{1}(s)+B_{20} s^{\alpha_{2}-\alpha_{1}}+\sum_{k>\alpha_{2}-\alpha_{1}} a_{k} s^{k}\right\} \\
& \text { with } B_{20}=-\frac{c_{20}}{\alpha_{1}}\left(c^{-\frac{1}{\alpha_{1}}}\right)^{\alpha_{2}-\alpha_{1}} \\
& \text { where } \quad Q_{1}(s)=B_{11} s^{d_{1}}+B_{12} s^{2 d_{1}}+\cdots+B_{1, p_{1}} s^{p_{1} d_{1}}
\end{aligned}
$$

such that the $B_{1 i}$ are complex numbers for $1 \leq i \leq p_{1}$.

REMARK. Next, in preparation for the proof of Case (II), suppose by the induction assumption on the integer $j$ with $1 \leq j \leq r$ that Step 1, Step 2, .., Step $j$ with $j \leq(r-1)$ were proved to be true. Then to prove that Step $(j+1)$ with $(j+1) \leq r$ is true, we may begin with $\phi(s)=\phi_{j}(s)$ which was already given by Step $j$, if necessary. Then it is very clear that the following sublemma by using the induction on the integer $p_{j+1}$, denoted by Sublemma 2, is just the replacement of Step $(j+1)$ of Lemma 3.4.2 which is needed to prove. Let us write down this sublemma. After then, we will prove Sublemma I and Sublemma 2, respectively.

Sublemma II. Let $\phi(s)$ be defined by $\phi_{j}(s)$,

$$
\begin{aligned}
\phi_{j}(s)= & c^{-\frac{1}{\alpha_{1}}} s\left\{1+Q_{1} s+s^{\alpha_{2}-\alpha_{1}}\left(B_{20}+Q_{2}(s)\right)+s^{\alpha_{3}-\alpha_{1}}\left(B_{30}+Q_{3}(s)\right)\right. \\
& \left.+\cdots+s^{\alpha_{j}-\alpha_{1}}\left(B_{j 0}+Q_{j}(s)\right)+B_{j+1,0} s^{\alpha_{j+1}-\alpha_{1}}+\sum_{k>\alpha_{j+1}-\alpha_{1}} a_{k} s^{k}\right\} .
\end{aligned}
$$

Note that $p_{j+1}$ is the nonnegative integer such that

$$
p_{j+1} d_{j+1}<\alpha_{j+2}-\alpha_{j+1}<\left(p_{j+1}+1\right) d_{j+1} .
$$

Then there are two subcases: $p_{j+1}=0$ and $p_{j+1}>0$.

Subcase (II-1) Let $p_{j+1}=0$.

(i) $s^{\alpha_{j+2}-\alpha_{1}} \in E_{j+2}^{*}(\phi(s))$, but $s^{\alpha_{j+2}-\alpha_{j+1}} \in E_{i}^{*}(\phi(s))$ for all $i \neq j+2$.

(ii) $s^{\alpha_{j+2}-\alpha_{1}} \in H^{*}(\phi(s))$ with coefficient $-\frac{1}{\alpha_{1}} c_{j+2,0}\left(c^{-\frac{1}{\alpha_{1}}}\right)^{\alpha_{j}-\alpha_{1}}$.

(iii) Then $\phi(s)$ can be defined by $\phi_{j+1}(s)$,

$$
\begin{aligned}
\phi_{j+1}(s)= & c^{-\frac{1}{\alpha_{1}}} s\left\{1+Q_{1}(s)+s^{\alpha_{2}-\alpha_{1}}\left(B_{20}+Q_{2}(s)\right)+\cdots\right. \\
& \left.+s^{\alpha_{j+1}-\alpha_{1}}\left(B_{j+1,0}+Q_{j+1}(s)\right)+B_{j+2,0} s^{\alpha_{j+2}-\alpha_{1}}+\sum_{k>\alpha_{j+2}-\alpha_{1}} a_{k} s^{k}\right\} \\
& \text { with } \quad B_{j+2,0}=-\frac{1}{\alpha_{1}} c_{j+2,0}\left(c^{-\frac{1}{\alpha_{1}}}\right)^{\alpha_{j+2}-\alpha_{1}} \\
& \text { where } \quad Q_{j+1}(s) \text { is identically zero. }
\end{aligned}
$$

Subcase (II-2) Let $p_{j+1}>0$. In this case, instead of proving Step $(j+1)$ directly,

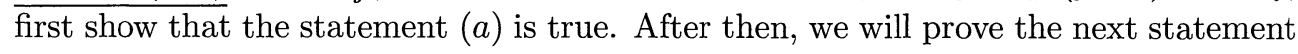


(b) is true. Note that the statement $(b)$ and the statement of Step $(j+1)$ are the same.

(a) For any integer $\tau=0,1,2, \ldots, p_{j+1}, \phi(s)$ can be defined by $\phi_{j+1, \tau}(s)$ as follows:

$$
\begin{aligned}
\phi_{j+1, \tau}(s)= & c^{-\frac{1}{\alpha_{1}}} s\left\{1+Q_{1}(s)+s^{\alpha_{2}-\alpha_{1}}\left(B_{20}+Q_{2}(s)\right)+\cdots+s^{\alpha_{j}-\alpha_{1}}\left(B_{j 0}+Q_{j}(s)\right)\right. \\
& \left.+s^{\alpha_{j+1}-\alpha_{1}}\left(B_{j+1,0}+Q_{j+1, \tau}^{*}(s)\right)+\sum_{k>\alpha_{j+1}-\alpha_{1}+\tau d_{j+1}} a_{k} s^{k}\right\} \\
& \text { where } \quad Q_{j+1, \tau}^{*}(s)=B_{j+1,1}^{*} s^{d_{j+1}}+B_{j+1,2}^{*} s^{2 d_{j+1}}+\cdots+B_{j+1, \tau}^{*} s^{\tau d_{j+1}}
\end{aligned}
$$

such that he $B_{j+1, i}^{*}$ are complex numbers for $1 \leq i \leq \tau$.

If $\tau=0$, note that $Q_{j+1, \tau}^{*}(s)$ is zero.

(b) In particular, if $\tau=p_{j+1}$, then we may begin with $\phi(s)=\phi_{j+1, p_{j+1}(s)}$. The remaining part of Step $(j+1)$ just follows from

(i) $s^{\alpha_{j+2}-\alpha_{1}} \in E_{j+2}^{*}(\phi(s))$, but $s^{\alpha_{j+2}-\alpha_{1}} \notin E_{i}^{*}(\phi(s))$ for all $i \neq 2$.

(ii) $s^{\alpha_{j+2}-\alpha_{1}} \in H^{*}(\phi(s))$ with coefficient $-\frac{c_{j+2,0}}{\alpha_{1}}\left(c^{-\frac{1}{\alpha_{1}}}\right)^{\alpha_{j+2}-\alpha_{1}}$.

(iii) Then $\phi(s)$ can be defined by $\phi_{j+1}(s)$,

$$
\begin{aligned}
\phi_{j+1}(s)= & -\frac{1}{\alpha_{1}} s\left\{1+Q_{1}(s)+s^{\alpha_{2}-\alpha_{1}}\left(B_{20}+Q_{2}(s)\right)+\cdots\right. \\
& \left.+s^{\alpha_{j+1}-\alpha_{1}}\left(B_{j+1,0}+Q_{j+1}(s)\right)+B_{j+2,0} s^{\alpha_{j+2}-\alpha_{1}}+\sum_{k>\alpha_{j+2}-\alpha_{1}} a_{k} s^{k}\right\} \\
& \text { with } \quad B_{j+2,0}=-\frac{c_{j+2,0}}{\alpha_{1}}\left(c^{-\frac{1}{\alpha_{1}}}\right)^{\alpha_{j+2}-\alpha_{1}} \\
& \text { where } Q_{j+1}(s)=B_{j+1,1} s^{d_{j+1}}+B_{j+1,2} s^{2 d_{j+1}}+\cdots+B_{j+1, p_{j+1}} s^{p_{j+1} d_{j+1}}
\end{aligned}
$$

such that the $B_{j+1, i}$ are complex numbers for $1 \leq i \leq p_{j+1}$.

Proof of Sublemma I. Let $t=\phi(s)=c^{-\frac{1}{\alpha_{1}}} s(1+H(t))^{-\frac{1}{\alpha_{1}}}$.

Subcase (I-1) To prove $\left(3.4 .26^{*}\right)$, recall by $(3.4 .17),(3.4 .18),(3.4 .20)$ and $(3.4 .21)$ that

$$
\begin{aligned}
\phi(s) & =c^{-\frac{1}{\alpha_{1}}} s\left(1+H^{*}(t)\right) \\
& =c^{-\frac{1}{\alpha_{1}}} s\left\{1+E_{2}^{*}(t)+E_{3}^{*}(t)+\cdots+E_{r+1}^{*}(t)\right\}
\end{aligned}
$$

because by (3.4.18) and (3.4.20) $p_{1}=0$ implies that $E_{1}^{*}(t)=D_{1}^{*}(t)$ is identically zero. To prove $\left(3.4 .26^{*}\right)$, it is needed just to compute $E_{k}^{*}(\phi(s))$ with $\phi(s)=c^{-\frac{1}{\alpha_{1}}} s(1+$ $\left.\sum_{k>0}^{\infty} a_{k} s^{k}\right)$.

Note by (3.4.18) and (3.4.20) that

$$
\operatorname{mult}\left(E_{i}^{*}(t), 0\right)=\alpha_{i}-\alpha_{1}>\operatorname{mult}\left(E_{2}^{*}(t), 0\right)=\alpha_{2}-\alpha_{1} \quad \text { for each } i=3,4, \ldots, r+3 .
$$

So, to get $\left(3.4 .26^{*}\right)$ with (i), (ii) and (iii), it remains to show that

$$
E_{2}^{*}(\phi(s))=-\frac{c_{20}}{\alpha_{1}}\left(c^{-\frac{1}{\alpha_{1}}}\right)^{\alpha_{2}-\alpha_{1}} s^{\alpha_{2}-\alpha_{1}}+\sum_{k>\alpha_{2}-\alpha_{1}} \mu_{k} s^{k}
$$


where the $\mu_{k}$ are complex numbers. Note that

$$
E_{2}^{*}(t)=t^{\alpha_{2}-\alpha_{1}}\left(-\frac{c_{20}}{\alpha_{1}}+D_{2}^{*}(t)\right)
$$

where $E_{2}^{*}(t)$ are defined by (3.4.18) and (3.4.20) and $D_{2}^{*}(0)=0$. So,

$$
\begin{aligned}
& E_{2}^{*}(\phi(s)) \\
= & \left\{c^{-\frac{1}{\alpha_{1}}} s\left(1+\sum_{k>0} a_{k} s^{k}\right)\right\}^{\alpha_{2}-\alpha_{1}}\left\{-\frac{c_{20}}{\alpha_{1}}+D_{2}^{*}(\phi(s))\right\} \\
= & -\frac{c_{20}}{\alpha_{1}}\left(c^{-\frac{1}{\alpha_{1}}}\right)^{\alpha_{2}-\alpha_{1}} s^{\alpha_{2}-\alpha_{1}} \\
& +\left\{\text { nonzero monomials of degree }>\alpha_{2}-\alpha_{1} \text { in the variable } s\right\} .
\end{aligned}
$$

Thus, the proof of this subcase with $\left(3.4 .26^{*}\right)$ is done.

$\underline{\text { Subcase (I-2) }}$ Let $p_{1}>0$ and $\phi(s)=c^{-\frac{1}{\alpha_{1}}} s\left(1+\sum_{k>0} a_{k} s^{k}\right)$.

(a) The proof of the representation of $\phi_{1, \tau}(s)$ in $\left(3.4 .27^{*}\right)$ will be induction on the integer $\tau$ with $0 \leq \tau \leq p_{1}$.

(a1) Assume that $\tau=0$. Note by $\left(3.4 .27^{*}\right)$ that

$$
\phi_{1,0}(s)=c^{-\frac{1}{\alpha}} s\left\{1+\sum_{k>0} a_{k} s^{k}\right\}
$$

which is trivial to show, because $Q_{1,0}^{*}(s)=0$.

(a2) Suppose that if $\tau=j<p_{1}$, then $\phi(s)$ can be defined by $\phi_{1, j}(s)$ in $\left(3.4 .27^{*}\right)$, that is,

$$
\begin{aligned}
& \phi_{1, j}(s)=c^{-\frac{1}{\alpha_{1}}} s\left\{1+Q_{1, j}^{*}(s)+\sum_{k>j d_{1}} a_{k} s^{k}\right\} \\
& \text { where } \quad Q_{1, j}^{*}(s)=B_{11}^{*} s^{d_{1}}+B_{12}^{*} s^{2 d_{1}}+\cdots+B_{1 j}^{*} s^{j d_{1}}
\end{aligned}
$$

such that the $B_{1 i}^{*}$ are complex numbers for $1 \leq i \leq j$.

To prove $\left(3.4 .27^{*}\right)$ with $\tau=j+1$, we may need to compute $H^{*}\left(\phi_{1, j}(s)\right)$, when we substitute $t=\phi(s)$ by $\phi_{1, j}(s)$ in the following equation

$$
\begin{aligned}
\phi(s) & =c^{-\frac{1}{\alpha_{1}}} s\left(1+H^{*}(t)\right) \\
& =c^{-\frac{1}{\alpha_{1}}} s\left\{1+E_{1}^{*}(t)+E_{2}^{*}(t)+\cdots+E_{r+1}^{*}(t)\right\} .
\end{aligned}
$$

After then, consider

$$
\begin{aligned}
\phi(s) & =c^{-\frac{1}{\alpha_{1}}} s\left(1+H^{*}\left(\phi_{1, j}(s)\right)\right. \\
& =c^{-\frac{1}{\alpha_{1}}} s\left\{1+E_{1}^{*}\left(\phi_{1, j}(s)\right)+E_{2}^{*}\left(\phi_{1, j}(s)\right)+\cdots+E_{r+1}^{*}\left(\phi_{1, j}(s)\right)\right\} .
\end{aligned}
$$

Since $p_{1}$ is positive, then in order to prove $\left(3.4 .27^{*}\right)$ with $\tau=j+1$, it may be assumed that

mult $\left(E_{1}^{*}(t), 0\right) \leq p_{1} d_{1} \quad$ for the brevity of computation, 
otherwise there is nothing to prove, because if $\operatorname{mult}\left(E_{1}^{*}(t), 0\right)>p_{1} d_{1}$, then $E_{1}^{*}(t)$ is identically zero and $\operatorname{mult}\left(E_{i}^{*}(t), 0\right) \geq\left(\alpha_{2}-\alpha_{1}\right)>p_{1} d_{1} \geq(j+1) d_{1}$ for $i=2,3, \ldots, r+1$.

Now, to prove $\left(3.4 .27^{*}\right)$ when $\tau=j+1$, we just need the following claims:

(i) If $s^{\mu} \in E_{1}^{*}\left(\phi_{1, j}(s)\right)$ with $\mu \leq(j+1) d_{1} \leq p_{1} d_{1}$, then $\mu$ is divisible by $d_{1}$.

(ii) If $s^{\sigma} \in E_{u}^{*}\left(\phi_{1, j}(s)\right)$ with $2 \leq u \leq(r+1)$, then $\sigma \geq \alpha_{2}-\alpha_{1}>p_{1} d_{1} \geq(j+1) d_{1}$. Since $\operatorname{mult}\left(E_{u}^{*}(t), 0\right)=\alpha_{i}-\alpha_{1} \geq \alpha_{2}-\alpha_{1}>p_{1} d_{1} \geq(j+1) d_{1}$ for any $u \geq 2$, then for proof of the claim, it remains to show (i) of the claim. Note by (3.4.18) that

$$
E_{1}^{*}(t)=D_{1}^{*}(t)=A_{11} t^{d_{1}}+A_{12} t^{2 d_{1}}+\cdots+A_{1 p_{1}} t^{p_{1} d_{1}} .
$$

For any term $A_{1 \xi} t^{\xi d_{1}} \in E_{1}^{*}(t)$ with $1 \leq \xi \leq p_{1}$ in (3.4.41), we substitute $t$ by $\phi_{1, j}(s)$ of (3.4.38). Then, $t^{\xi d_{1}}=\left(\phi_{1 j}(s)\right)^{\xi d_{1}}$ becomes

$$
\left(\phi_{1, j}(s)\right)^{\xi d_{1}}=\left\{c^{-\frac{1}{\alpha_{1}}} s\left(1+Q_{1, j}^{*}(s)+\sum_{k>j d_{1}} a_{k} s^{k}\right)\right\}^{\xi d_{1}} .
$$

Whenever $s^{\mu} \in\left(\phi_{1, j}(s)\right)^{\xi d_{1}}$ of (3.4.42) such that $\mu \leq(j+1) d_{1}$, then $\mu$ is divisible by $d_{1}$ by Lemma 3.4.1 because $\xi$ is positive, and also because if $s^{k} \in Q_{1, j}^{*}(s)$ then the exponent $k$ may be considered as a multiple of $d_{1}$ in $Q_{1, j}^{*}(s)$ whether or not $Q_{1, j}^{*}(s)$ is identically zero. Since $\operatorname{mult}\left(H^{*}(t)-E_{1}^{*}(t), 0\right)=\alpha_{2}-\alpha_{1}$, then we may assume that $E_{1}^{*}\left(\phi_{1, j}(s)\right)$ has a monomial $s^{(j+1) d_{1}}$ whether or not its coefficient is zero. Therefore, $\phi(s)$ can be defined by $\phi_{1, j+1}(s)$ such that

$$
\begin{array}{r}
\phi_{1, j+1}(s)=c^{-\frac{1}{\alpha_{1}}} s\left\{1+Q_{1, j+1}^{*}+\sum_{k>(j+1) d_{1}} a_{k} s^{k}\right\} \\
Q_{1, j+1}^{*}(s)=B_{11}^{*} s^{d_{1}}+\cdots+B_{1, j+1}^{*} s^{(j+1) d_{1}}
\end{array}
$$

such that the $B_{1 i}^{*}$ are complex numbers for $1 \leq i \leq j+1$.

(b) Let $p_{1}$ be the positive integer such that $p_{1} d_{1}<\alpha_{2}-\alpha_{1}<\left(p_{1}+1\right) d_{1}$. To prove $\left(3.4 .28^{*}\right)$, consider

$$
\begin{aligned}
\phi(s) & =c^{-\frac{1}{\alpha_{1}}} s\left(1+E_{1}^{*}(t)+\cdots+E_{r+1}^{*}(t)\right) \quad \text { with } \\
t=\phi_{1, p_{1}}(s) & =c^{-\frac{1}{\alpha_{1}}} s\left(1+Q_{1, p_{1}}^{*}(s)+\sum_{k>p_{1} d_{1}} a_{k} s^{k}\right)
\end{aligned}
$$

where

$$
Q_{1, p_{1}}^{*}(s)=B_{11}^{*} s^{d_{1}}+\cdots+B_{1, p_{1}}^{*} s^{p_{1} d_{1}}
$$

such that the $B_{1, i}^{*}$ are complex numbers. Since

$$
\begin{aligned}
\left(p_{1}+1\right) d_{1} & >\operatorname{mult}\left(E_{2}^{*}(t), 0\right)=\alpha_{2}-\alpha_{1}>p_{1} d_{1} \quad \text { and } \\
\operatorname{mult}\left(E_{i}^{*}(t), 0\right) & >\operatorname{mult}\left(E_{2}^{*}(t), 0\right) \quad \text { for } i \geq 3,
\end{aligned}
$$

first it is needed to compute $E_{1}^{*}\left(\phi_{1, p_{1}}(s)\right)$ and $E_{2}^{*}\left(\phi_{1, p_{1}}(s)\right)$ instead of $H^{*}\left(\phi_{1, p_{1}}(s)\right)$.

From (3.4.41), recall that

$$
E_{1}^{*}(t)=D_{1}^{*}(t)=A_{11} t^{d_{1}}+\cdots+A_{1, p_{1}}^{p_{1} d_{1}}
$$


As in the proof of (3.4.43), for any $t^{\xi d_{1}} \in E_{1}^{*}(t)$ with $1 \leq \xi \leq p_{1}$,

$$
\begin{aligned}
t^{\xi d_{1}} & =\left(\phi_{1, p_{1}}(s)\right)^{\xi d_{1}} \\
& =\left\{c^{-\frac{1}{\alpha_{1}}} s\left(1+Q_{1, p_{1}}^{*}(s)+\sum_{k>p_{1} d_{1}} a_{k} s^{k}\right)\right\}^{\xi d_{1}} .
\end{aligned}
$$

Whenever $s^{\mu} \in\left(\phi_{1, p_{1}}(s)\right)^{\xi d_{1}}$ of (3.4.45) such that $\mu \leq\left(p_{1}+1\right) d_{1}$, then $\mu$ is divisible by $d_{1}$, which can be proved by the same way as we have done in the proof of $(a)$. Because $\xi$ was chosen arbitrary with $1 \leq \xi \leq p_{1}$, we get

$$
\begin{aligned}
E_{1}^{*}\left(\phi_{1, p_{1}(s)}\right) & =Q_{1}^{*}(s)+\sum_{k>\left(p_{1}+1\right) d_{1}} b_{k} s^{k} \text { with } \\
Q_{1}^{*}(s) & =B_{11} s^{d_{1}}+B_{12} s^{2 d_{1}}+\cdots+B_{1, p_{1}+1} s^{\left(p_{1}+1\right) d_{1}}
\end{aligned}
$$

such that the $B_{1 i}$ are complex numbers for $1 \leq i \leq p_{1}+1$. Since $E_{2}^{*}(t)=t^{\alpha_{2}-\alpha_{1}}\left(-\frac{c_{20}}{\alpha_{1}}+\right.$ $\left.D_{2}^{*}(t)\right)$ implies that

$$
p_{1} d_{1}<\operatorname{mult}\left(E_{2}^{*}(t), 0\right)=\alpha_{2}-\alpha_{1}<\left(p_{1}+1\right) d_{1},
$$

it is clear that $E_{2}^{*}\left(\phi_{1, j+1}(s)\right)$ can be written in the form

$$
\begin{aligned}
E_{2}^{*}\left(\phi_{1, j+1}(s)\right)= & \left\{\left(c^{-\frac{1}{\alpha_{1}}} s\right)^{\alpha_{2}-\alpha_{1}}+\text { monomials of degree }>\alpha_{2}-\alpha_{1} \text { in the variable } s\right\} \\
& \times\left\{-\frac{c_{20}}{\alpha_{1}}+\text { monomials of degree }>0 \text { in the variable } s\right\} \\
= & s^{\alpha_{2}-\alpha_{1}}\left\{-\frac{c_{20}}{\alpha_{1}}\left(c^{-\frac{1}{\alpha_{1}}}\right)^{\alpha_{2}-\alpha_{1}}+\sum_{k>0} \mu_{k} s^{k}\right\}
\end{aligned}
$$

where the $\mu_{k}$ are complex numbers. Note that

$$
\operatorname{mult}\left(E_{i}^{*}(t), 0\right)=\alpha_{i}-\alpha_{1}>\alpha_{2}-\alpha_{1} \quad \text { for any } i \geq 3 .
$$

Now, substitute (3.4.44) by (3.4.46), (3.4.47) and (3.4.48). Then, it can be proved that $\phi(s)$ is written in the form $\left(3.4 .28^{*}\right)$ which satisfies the properties (i), (ii) and (iii). Thus, the proof of Sublemma I is finished.

\section{Proof of Sublemma II.}

Subcase (II-1) Let $p_{j+1}=0$. First, recall by $(3.4 .17),(3.4,18),(3.4 .20)$ and (3.4.21), and by $\left(3.4 .29^{*}\right)$ from the induction assumption that

$$
\begin{aligned}
\phi_{j}(s)= & c^{-\frac{1}{\alpha_{1}}} s\left\{1+Q_{1}(s)+s^{\alpha_{2}-\alpha_{1}}\left(B_{20}+Q_{2}(s)\right)+s^{\alpha_{3}-\alpha_{1}}\left(B_{30}+Q_{3}(s)\right)\right. \\
& \left.+\cdots+s^{\alpha_{j}-\alpha_{1}}\left(B_{j 0}+Q_{j}(s)\right)+B_{j+1,0} s^{\alpha_{j+1}-\alpha_{1}}+\sum_{k>\alpha_{j+1}-\alpha_{1}} a_{k} s^{k}\right\} \\
& \text { with } B_{j+1,0}=-\frac{c_{j+1,0}}{\alpha_{1}}\left(c^{-\frac{1}{\alpha_{1}}}\right)^{\alpha_{2}-\alpha_{1}} .
\end{aligned}
$$


Also, note that

$$
\begin{aligned}
\phi(s) & =c^{-\frac{1}{\alpha_{1}}} s\left(1+H^{*}(t)\right) \\
& =c^{-\frac{1}{\alpha_{1}}} s\left(1+E_{1}^{*}(t)+E_{2}^{*}(t)+\cdots+E_{r+1}^{*}(t)\right)
\end{aligned}
$$

To prove $\left(3.4 .30^{*}\right)$, it is needed to compute $H^{*}\left(\phi_{j}(s)\right)$ in $(3.4 .50)$, by substituting $\phi_{j}(s)$ of (3.4.49) for $t$. For computation of $E_{i}^{*}\left(\phi_{j}(s)\right)$, recall by (3.4.20) that

$$
\begin{aligned}
& E_{1}^{*}(t)=D_{1}^{*}(t) \\
& E_{i}^{*}(t)=t^{\alpha_{i}-\alpha_{1}}\left(-\frac{c_{i 0}}{\alpha_{1}}+D_{i}^{*}(t)\right) \quad \text { for } 2 \leq i \leq r+1 \\
& H^{*}(t)=E_{1}^{*}(t)+E_{2}^{*}(t)+\cdots+E_{r+1}^{*}(t) .
\end{aligned}
$$

Since

$$
\operatorname{mult}\left(E_{i}^{*}(t), 0\right)>\operatorname{mult}\left(E_{j+2}^{*}(t), 0\right)=\alpha_{j+2}-\alpha_{1} \quad \text { for any } i=j+3, \ldots, r+1,
$$

and $\phi(0)=0$, then it is obvious that $s^{\alpha_{j+2}-\alpha_{1}} \notin E_{i}^{*}(\phi(s))$. Also, it can be easily shown that $\operatorname{mult}\left(E_{j+2}^{*}(\phi(s)), 0\right)=\alpha_{j+2}-\alpha_{1}$ and $s^{\alpha_{j+2}-\alpha_{1}} \in E_{j+2}^{*}(\phi(s))$ with its coefficient $c^{-\frac{1}{\alpha_{1}}\left(\alpha_{j+2}-\alpha_{1}\right)} \frac{c_{j+2,0}}{-\alpha_{1}}$ as we have done in the proof of (3.4.48).

So,to prove $\left(3.4 .30^{*}\right)$ with (i), (ii) and (iii), it is enough to show that

$$
s^{\alpha_{j+2}-\alpha_{1}} \notin E_{k}^{*}(\phi(s)) \text { for } k=1,2, \cdots, j+1,
$$

because $p_{j+1}=0$ with $0<\alpha_{j+2}-\alpha_{j+1}<d_{j+1}$ implies that $Q_{j+1}(s)$ is identically zero.

First, compute $E_{1}^{*}\left(\phi_{j}(s)\right)$ if exists where $E_{1}^{*}(t)=D_{1}^{*}(t)=A_{11} t^{d_{1}}+\cdots+A_{1 p_{1}} t^{p_{1} d_{1}}$ by (3.4.41). By the same method as in the proof of (3.4.42), for any $A_{1 \xi} t^{\xi d_{1}} \in E_{1}^{*}(t)$ with $1 \leq \xi \leq p_{1}, t^{\xi d_{1}}=\left(\phi_{j}(s)\right)^{\xi d_{1}}$ becomes

$$
\begin{aligned}
\left(\phi_{j}(s)\right)^{\xi d_{1}}= & {\left[c ^ { - \frac { 1 } { \alpha _ { 1 } } } s \left\{1+Q_{1}(s)+s^{\alpha_{2}-\alpha_{1}}\left(B_{20}+Q_{2}(s)\right)+\cdots\right.\right.} \\
& \left.\left.+s^{\alpha_{j}-\alpha_{1}}\left(B_{j 0}+Q_{j}(s)\right)+B_{j+1,0} s^{\alpha_{j+1}-\alpha_{1}}+\sum_{k>\alpha_{j+1}-\alpha_{1}} a_{k} s^{k}\right\}\right]^{\xi d_{1}} .
\end{aligned}
$$

So, whenever $s^{\mu} \in\left(\phi_{j}(s)\right)^{\xi d_{1}}$ of (3.4.52) such that $\mu \leq \alpha_{j+1}-\alpha_{1}+\xi d_{1}$, then $\mu$ is divisible by $d_{j+1}$, but there is a nonzero monomial $s^{\alpha_{j+1}-\alpha_{1}+\xi d_{1}} \in\left(\phi_{j}(s)\right)^{\xi d_{1}}$ such that the exponent $\alpha_{j+1}-\alpha_{1}+\xi d_{1}$ is not divisible by $d_{j}$ by Lemma 3.4.1 because $B_{j+1,0} \neq 0$.

In particular, if $s^{\mu} \in\left(\phi_{j}(s)\right)^{\xi d_{1}}$ with $\xi=1$ and $\mu$ is not divisible by $d_{j}$ such that $\mu \leq \alpha_{j+1}-\alpha_{1}+d_{1}$, then $\mu=\alpha_{j+1}-\alpha_{1}+d_{1}$.

By construction of $p_{j+1}, p_{j+1}=0$ and $d_{j+1}<d_{1}$ imply that

$$
\begin{aligned}
p_{j+1} d_{j+1} & <\alpha_{j+2}-\alpha_{j+1}<\left(p_{j+1}+1\right) d_{j+1}, \quad \text { or } \\
0 & <\alpha_{j+2}-\alpha_{j+1}<d_{j+1}, \quad \text { and } \\
\alpha_{j+1}-\alpha_{1} & <\alpha_{j+2}-\alpha_{1}<\alpha_{j+1}-\alpha_{1}+d_{j+1}<\alpha_{j+2}-\alpha_{1}+d_{1} .
\end{aligned}
$$

So, $s^{\alpha_{j+2}-\alpha_{1}} \notin\left(\phi_{j}(s)\right)^{\xi d_{1}}$ because $\alpha_{j+2}-\alpha_{1} \leq \alpha_{j+1}-\alpha_{1}+\xi d_{j+1}$ and $\alpha_{j+2}-\alpha_{1}$ can not be divisible by $d_{j+1}$. Since $\xi$ was chosen arbitrary, then it can be proved that $s^{\alpha_{j+2}-\alpha_{1}} \notin E_{1}^{*}(\phi(s))$ with $\alpha_{j+2}-\alpha_{1}<d_{1}$. 
Next, compute

$(* *)$

$E_{i}^{*}\left(\phi_{j}(s)\right) \quad$ for $2 \leq i \leq j+1$

where $E_{i}^{*}(t)=t^{\alpha_{i}-\alpha_{1}}\left(-\frac{c_{i 0}}{\alpha_{1}}+D_{i}^{*}(t)\right) \quad$ and $D_{i}^{*}(t)=A_{i 1} t^{d_{i}}+\cdots+A_{i p_{i}} t^{p_{i} d_{i}}$.

Since $p_{j+1}=0$, then note that $D_{j+1}^{*}(t)$ is identically zero. As in the proof of (3.4.52), we are going to compute from $-\frac{c_{i 0}}{\alpha_{1}} t^{\alpha_{i}-\alpha_{1}}$,

$$
\begin{aligned}
\left(\phi_{j}(s)\right)^{\alpha_{i}-\alpha_{1}} & =\left[c ^ { - \frac { 1 } { \alpha _ { 1 } } } s \left\{1+Q_{1}(s)+s^{\alpha_{2}-\alpha_{1}}\left(B_{20}+Q_{2}(s)\right)+\cdots\right.\right. \\
& \left.\left.+s^{\alpha_{j}-\alpha_{1}}\left(B_{j 0}+Q_{j}(s)\right)+B_{j+1,0} s^{\alpha_{j+1}-\alpha_{1}}+\sum_{k>\alpha_{j+1}-\alpha_{1}} a_{k} s^{k}\right\}\right]^{\alpha_{i}-\alpha_{1}}
\end{aligned}
$$

instead of $E_{i}^{*}\left(\phi_{j}(s)\right)$.

Let $i$ be fixed. Since $B_{j+1,0} \neq 0$, then by Lemma 3.4 .1 we have the following properties:

(3.4.53) Whenever $s^{\mu} \in\left(\phi_{j}(s)\right)^{\alpha_{i}-\alpha_{1}}$ such that $\mu \leq \alpha_{i}-\alpha_{1}+\alpha_{j+1}-\alpha_{1}$, where $\phi_{j}(s)$ was defined by (3.4.49), then $\mu$ is divisible by $d_{j+1}$, but there is a nonzero monomial $s^{\alpha_{i}-\alpha_{1}+\alpha_{j+1}-\alpha_{1}} \in\left(\phi_{j}(s)\right)^{\alpha_{i}-\alpha_{1}}$ such that the exponent $\alpha_{i}-\alpha_{1}+\alpha_{j+1}-\alpha_{1}$ is not divisible by $d_{j}$.

(3.4.54) Because $\alpha_{i}-\alpha_{1} \geq d_{i}=\operatorname{gcd}\left(n, \alpha_{1}, \alpha_{2}, \cdots, \alpha_{i}\right)$ and $0<\alpha_{j+2}-\alpha_{j+1}<d_{j+1}$ with $p_{j+1}=0, \alpha_{i}-\alpha_{1}+\alpha_{j+1}-\alpha_{1} \geq \alpha_{j+1}-\alpha_{1}+d_{i} \geq \alpha_{j+1}-\alpha_{1}+d_{j+1}>\alpha_{j+2}-\alpha_{1}$ for $2 \leq i \leq j+1$.

Then, we claim that $s^{\alpha_{j+2}-\alpha_{1}} \notin\left(\phi_{j}(s)\right)^{\alpha_{i}-\alpha_{1}}$. To prove the claim, assume the contrary. Now, since $s^{\alpha_{j+2}-\alpha_{1}} \in\left(\phi_{j}(s)\right)^{\alpha_{i}-\alpha_{1}}$, then $\alpha_{j+2}-\alpha_{1}$ must divisible by $d_{j+1}$ because of (3.4.53) and (3.4.54). It would be a contradiction, because $\alpha_{j+2}-\alpha_{1}$ cannot not be divisible by $d_{j+2}$. Thus, we proved that $s^{\alpha_{j+2}-\alpha_{1}} \notin\left(\phi_{j}(s)\right)^{\alpha_{i}-\alpha_{1}}$. Similarly, we can show that $s^{\alpha_{j+2}-\alpha_{1}}$ does not belong to $\left.\left(\phi_{j}(s)\right)\right)^{\alpha_{i}-\alpha_{1}+d_{i}}$, and does not belong to $E_{i}^{*}\left(\phi_{j}(s)\right)$, either where $E_{i}^{*}(t)$ was defined by $(* *)$.

Summarizing the above computations, we proved the followings:

(a) $s^{\alpha_{j+2}-\alpha_{1}} \in E_{j+2}^{*}\left(\phi_{j}(s)\right)$ with its coefficient $B_{j+2,0}=-\frac{1}{\alpha_{1}} c_{j+2,0} c^{-\frac{1}{\alpha_{1}}\left(\alpha_{j+2}-\alpha_{1}\right)}$ and $s^{\alpha_{j+2}-\alpha_{1}} \notin E_{i}^{*}\left(\phi_{j}(s)\right)$ for all $i \neq j+2$.

(b) By $(a), \phi(s)$ can be written in the form

$$
\begin{aligned}
\phi_{j+1}(s)= & c^{-\frac{1}{\alpha_{1}}} s\left\{1+Q_{1}(s)+s^{\alpha_{2}-\alpha_{1}}\left(B_{20}+Q_{2}(s)\right)+\cdots\right. \\
& +s^{\alpha_{j+1}-\alpha_{1}}\left(B_{j+1,0}+Q_{j+1,0}(s)\right)+B_{j+2,0} s^{\alpha_{j+2}-\alpha_{1}} \\
& \left.+\sum_{k>\alpha_{j+2}-\alpha_{1}} a_{k} s^{k}\right\} \text { with } \\
& B_{j+2,0}=-\frac{1}{\alpha_{1}} c_{j+2,0} c^{-\frac{1}{\alpha_{1}}\left(\alpha_{j+2}-\alpha_{1}\right)},
\end{aligned}
$$

where $Q_{j+1,0}(s)$ is identically zero. Thus, the proof of Subcase (II-1) is done. 
$\underline{\text { Subcase }(\mathrm{II}-2)}$ Let $p_{j+1}>0$ and $\phi_{j}(s)$ be defined by

$$
\begin{aligned}
\phi_{j}(s)= & c^{-\frac{1}{\alpha_{1}}} s\left\{1+Q_{1}(s)+s^{\alpha_{2}-\alpha_{1}}\left(B_{20}+Q_{2}(s)\right)\right. \\
& +s^{\alpha_{3}-\alpha_{1}}\left(B_{30}+Q_{3}(s)\right)+\cdots+s^{\alpha_{j}-\alpha_{1}}\left(B_{j 0}+Q_{j}(s)\right) \\
& \left.+B_{j+1,0} s^{\alpha_{j+1}-\alpha_{1}}+\sum_{k>\alpha_{j+1}-\alpha_{1}} a_{k} s^{k}\right\} .
\end{aligned}
$$

(a) The proof will be induction on the integer on $\tau$ with $0 \leq \tau \leq p_{j+1}$.

(a1) To prove that $\phi_{j+1,0}(s)$ has the desired form in (3.4.31) when $\tau=0$, it is clear that $\phi_{j+1,0}(s)=\phi_{j}(s)$ of $(3.4 .56)$ because $Q_{j+1,0}^{*}(s)$ of (3.4.31) is identically zero.

(a2) Suppose that if $\tau=e<p_{j+1}$, then $\phi(s)$ can be defined by $\phi_{j+1, e}(s)$ in (3.4.31):

$$
\begin{aligned}
\phi_{j+1, e}(s)= & c^{-\frac{1}{\alpha_{1}}} s\left\{1+Q_{1}(s)+s^{\alpha_{2}-\alpha_{1}}\left(B_{20}+Q_{2}(s)\right)+\cdots\right. \\
& +s^{\alpha_{j}-\alpha_{1}}\left(B_{j 0}+Q_{j}(s)\right)+s^{\alpha_{j+1}-\alpha_{1}}\left(B_{j+1,0}+Q_{j+1, e}^{*}(s)\right) \\
& \left.+\sum_{k>\alpha_{j+1}-\alpha_{1}+e d_{j+1}} a_{k} s^{k}\right\} \\
& \text { where } Q_{j+1, e}^{*}(s)=B_{j+1,1}^{*} s^{d_{j+1}}+B_{j+1,2}^{*} s^{2 d_{j+1}}+\cdots+B_{j+1, e}^{*} s^{e d_{j+1}}
\end{aligned}
$$

such that the $B_{j+1, i}^{*}$ are complex numbers for $1 \leq i \leq \tau=e$.

To prove (3.4.31) with $\tau=e+1 \leq p_{j+1}$, we may need to compute $H^{*}\left(\phi_{j, e}(s)\right)$. Recall that

$$
\begin{aligned}
\phi(s) & =c^{-\frac{1}{\alpha_{1}}} s\left(1+H^{*}(t)\right) \\
& =c^{-\frac{1}{\alpha_{1}}} s\left(1+E_{1}^{*}(t)+\cdots+E_{r+1}^{*}(t)\right) .
\end{aligned}
$$

By (3.4.57) and (3.4.58), it is enough to prove the following claim:

For any $s^{\mu} \in H^{*}\left(\phi_{j, e}(s)\right)$ such that $\mu \leq \alpha_{j+1}-\alpha_{1}+(e+1) d_{j+1}$ then $d_{j+1} \mid \mu$.

Note that $\alpha_{j+1}-\alpha_{1}+(e+1) d_{j+1}$ is divisible by $d_{j+1}$. To prove (3.4.59) of the above claim, compute $E_{i}^{*}\left(\phi_{j, e}(s)\right)$ by (i), (ii) and (iii), respectively as follows:

$$
\text { (i) } i=1 \text {, (ii) } 2 \leq i \leq j+1 \text { and (iii) } j+2 \leq i \leq r+1 \text {. }
$$

(i) First, compute $E_{1}^{*}\left(\phi_{j+1, e}(s)\right)$ where $E_{11}(t)=A_{11} t^{d_{1}}+\cdots+A_{1, p_{1}} t^{p_{1} d_{1}}$ is not identically zero. For any $A_{1 \xi} t^{\xi d_{1}} \in E_{1}^{*}(t)$ with $t=\phi_{j+1, e}(s)$ of (3.4.57), consider

$$
\left(\phi_{j+1, e}(s)\right)^{\xi d_{1}} \text {. }
$$

Whenever $s^{\mu} \in\left(\phi_{j+1, e}(s)\right)^{\xi d_{1}}$ of (3.4.57) such that $\mu \leq \xi d_{1}+\alpha_{j+1}-\alpha_{1}+e d_{j+1}$, then $d_{j+1} \mid \mu$ by Lemma 3.4.1, but for convenience of the proof we may assume that there is a nonzero monomial $s^{\xi d_{1}+\alpha_{j+1}-\alpha_{1}+e d_{j+1}} \in\left(\phi_{j+1, e}(s)\right)^{\xi d_{1}}$ by Lemma 3.4.1 because $B_{j+1}$ is not zero, whether or not $Q_{j+1, s}^{*}(s)$ is identically zero. In particular, 
if $\xi=1$, then

$$
\begin{aligned}
& \mu \leq d_{1}+\alpha_{j+1}-\alpha_{1}+e d_{j+1} \text { and also } \\
& d_{1}+\alpha_{j+1}-\alpha_{1}+e d_{j+1}>\alpha_{j+1}-\alpha_{1}+(e+1) d_{j+1},
\end{aligned}
$$

because $d_{1}>d_{j+1}$. In this case, we proved the claim.

(ii) To compute $E_{i}^{*}\left(\phi_{j+1, e}(s)\right)$ for $2 \leq i \leq j+1$, let $i$ be fixed. Recall that

$$
E_{i}^{*}(t)=t^{\alpha_{i}-\alpha_{1}}\left(-\frac{c_{i 0}}{\alpha_{1}}+D_{i}^{*}(t)\right)
$$

where

$$
D_{i}^{*}(t)=A_{i 1} t^{d_{i}}+\cdots+A_{i p_{i}} t^{p_{i} d_{i}} .
$$

To prove the claim, first compute $-\frac{c_{i 0}}{\alpha_{1}}\left(\phi_{j+1, e}(s)\right)^{\alpha_{i}-\alpha_{1}}$. If $s^{\mu} \in\left(\phi_{j+1, e}(s)\right)^{\alpha_{i}-\alpha_{1}}$ of (3.4.57) such that $\mu \leq \alpha_{i}-\alpha_{1}+\alpha_{j+1}-\alpha_{1}+e d_{j+1}$, then $d_{j+1} \mid \mu$ by Lemma 3.4.1 because $B_{j+1,0} \neq 0$. In particular, it may be assumed that there exists a nonzero monomial $s^{\nu} \in\left(\phi_{j+1, e}(s)\right)^{\alpha_{i}-\alpha_{1}}$ with $\nu=\alpha_{j+1}-\alpha_{1}+(e+1) d_{j+1}$ because $B_{j+1,0} \neq 0$ and then

$$
\begin{array}{r}
d_{i}=\operatorname{gcd}\left(n_{1}, \alpha_{1}, \ldots, \alpha_{i}\right) \geq d_{j+1}=\operatorname{gcd}\left(n_{1}, \alpha_{1}, \ldots, \alpha_{j+1}\right) \quad \text { for } 2 \leq i \leq j+1, \\
\alpha_{i}-\alpha_{1}+\alpha_{j+1}-\alpha_{1}+e d_{j+1} \geq \alpha_{j+1}-\alpha_{1}+(e+1) d_{j+1} .
\end{array}
$$

Thus, we proved the claim for $\left(\phi_{j, e}(s)\right)^{\alpha_{i}-\alpha_{1}}$. Also, using the same method as above, it can be easily shown that there is a nonzero monomial $s^{\nu} \in\left(\phi_{j+1, e}(s)\right)^{\alpha_{i}-\alpha_{1}} D_{i}^{*}\left(\phi_{j+1, e}(s)\right)$ with $\nu=\alpha_{j+1}-\alpha_{1}+(e+1) d_{j+1}$. So, the claim of this case can be proved by (4.63).

(iii) Since $\operatorname{mult}\left(E_{i}^{*}(t), 0\right) \geq \operatorname{mult}\left(E_{j+2}^{*}(t), 0\right)=\alpha_{j+2}-\alpha_{1}>\alpha_{j+1}-\alpha_{1}+(e+1) d_{j+1}$ for $j+2 \leq i \leq r+1$, then there is nothing to prove for the claim (3.4.59).

Therefore, the proof of the claim is done, and so we proved (a) of Subcase (II-2).

(b) Let $p_{j+1}$ be the positive integer such that $p_{j+1} d_{j+1}<\alpha_{j+2}-\alpha_{j+1}<\left(p_{j+1}+1\right) d_{j+1}$. For the proof, consider

$$
\phi(s)=c^{-\frac{1}{\alpha_{1}}} s\left\{1+E_{1}^{*}(t)+E_{2}^{*}(t)+\cdots+E_{r+1}^{*}(t)\right\} \text { and }
$$

$$
\begin{aligned}
\phi_{j+1, p_{j+1}}(s)= & c^{-\frac{1}{\alpha_{1}}} s\left\{1+Q_{1}(s)+s^{\alpha_{2}-\alpha_{1}}\left(B_{20}+Q_{2}(s)\right)+\cdots\right. \\
& +s^{\alpha_{j}-\alpha_{1}}\left(B_{j 0}+Q_{j}(s)\right)+s^{\alpha_{j+1}-\alpha_{1}}\left(B_{j+1,0}+Q_{j+1, p_{j+1}}^{*}(s)\right) \\
& \left.+\sum_{k>\alpha_{j+1}-\alpha_{1}+p_{j+1} d_{j+1}} a_{k} s^{k}\right\},
\end{aligned}
$$

where $\quad Q_{j+1, p_{j+1}}^{*}(s)=B_{j+1,1}^{*} s^{d_{j+1}}+B_{j+1,2}^{*} s^{2 d_{j+1}}+\cdots+B_{j+1, p_{j+1}}^{*} s^{p_{j+1} d_{j+1}}$,

such that the $B_{j+1, k}^{*}$ are complex numbers for $1 \leq k \leq p_{j+1}$. To prove (3.4.30*) with (i), (ii) and (iii), substitute $t$ by $\phi_{j+1, p_{j+1}}(s)$ and then compute $E_{i}^{*}(t)$. For the proof, it is enough to prove the following three cases:

(b1) $s^{\alpha_{j+2}-\alpha_{1}} \notin E_{i}^{*}\left(\phi_{j+1, p_{j+1}}(s)\right.$ for $j+3 \leq i \leq r+1$.

(b2) $s^{\alpha_{j+2}-\alpha_{1}} \in E_{i}^{*}\left(\phi_{j+1, p_{j+1}}(s)\right.$ for $i=j+2$.

(b3) $s^{\alpha_{j+2}-\alpha_{1}} \notin E_{i}^{*}\left(\phi_{j+1, p_{j+1}}(s)\right.$ for $1 \leq i \leq j+1$. 
Since $\operatorname{mult}\left(E_{i}^{*}(t), 0\right)>\operatorname{mult}\left(E_{j+1}^{*}(t), 0\right)=\alpha_{j+2}-\alpha_{1}$ for all $i=j+3, \ldots, r+1$ and $\phi(0)=0$, then it is clear that $s^{\alpha_{j+2}-\alpha_{1}} \notin E_{i}^{*}(\phi(s))$ for all $i=j+3, \ldots, r+$ 1 , and so (b1) can be easily shown. Next, consider $E_{j+2}^{*}\left(\phi_{j+1, p_{j+1}}(s)\right)$. Then it is clear by Lemma 3.4.1 that $s^{\alpha_{j+2}-\alpha_{1}}$ belongs to $E_{j+2}^{*}\left(\phi_{j+1, p_{j+1}}(s)\right)$ with its coefficient $B_{j+2,0}=-\frac{c_{j+2,0}}{\alpha_{1}}\left(c_{1}^{\frac{1}{\alpha_{1}}}\right)^{\alpha_{j+2}-\alpha_{1}}$ by the same method as we have done in the proof of (3.4.47). Thus, (b2) can be easily proved, too. Therefore, it remains to show that for $1 \leq i \leq j+1$

$$
s^{\alpha_{j+2}-\alpha_{1}} \notin E_{i}^{*}\left(\phi_{j+1, p_{j+1}}(s)\right),
$$

where $E_{i}^{*}(t)=t^{\alpha_{i}-\alpha_{1}}\left(-\frac{c_{i 0}}{\alpha_{1}}+D_{i}^{*}(t)\right)$ for $2 \leq i \leq r+1$. By the same technique as in the proof of (3.4.59) and Lemma 3.4.1, we can prove To prove (b3), compute $E_{i}^{*}\left(\phi_{j}+1(s)\right.$ ). First, compute $E_{1}^{*}\left(\phi_{j+1}(s)\right)$ where $E_{1}^{*}(t)=A_{11} t^{d_{1}}+\cdots+A_{1, p_{1}} t^{p_{1} d_{1}}$ is not identically zero. For any $A_{1 \xi} t^{\xi d_{1}} \in E_{1}^{*}(t)$ with $t=\phi_{j+1}$, consider

$$
\left(\phi_{j+1}(s)\right)^{\xi d_{1}}
$$

Whenever $s^{\mu} \in\left(\phi_{j+1}(s)\right)^{\xi d_{1}}$ of (3.4.66) such that $\mu \leq \xi d_{1}+\alpha_{j+1}-\alpha_{1}+p_{j+1} d_{j+1}$, then $d_{j+1} \mid \mu$ by Lemma 3.4 .1 because $B_{j+1,0} \neq 0$. Also, in particular, $d_{1}+\alpha_{j+1}-\alpha_{1}+$ $p_{j+1} d_{j+1}>\left(p_{j+1}+1\right) d_{j+1}+\alpha_{j+1}-\alpha_{1}>\alpha_{j+2}-\alpha_{1}$ and $\alpha_{j+2}-\alpha_{1}$ is not divisible by $d_{j+1}$. Since $\xi$ was arbitrarily chosen, we proved that $s^{\alpha_{j+2}-\alpha_{1}} \notin E_{1}^{*}\left(\phi_{j+1}(s)\right)$. Moreover, by the same method as we have just used in the proof of $s^{\alpha_{j+2}-\alpha_{1}} \notin$ $E_{1}^{*}\left(\phi_{j+1}(s)\right)$, we can show that $s^{\alpha_{j+2}-\alpha_{1}}$ does not belong to $E_{i}^{*}\left(\phi_{j+1}(s)\right)$ for $1 \leq i \leq$ $j+1$. Thus, the proof of $\left(3.4 .32^{*}\right)$ with (i), (ii) and (iii) can be finished. Therefore, the proof of Subcase (II-2) is done because $\alpha_{j+2}-\alpha_{1}<\left(p_{j+1}+1\right) d_{j+1}$. Thus, we finished the proof of Sublemma II.

Therefore, the proof of Lemma 3.4.2 is done, and so $t=\phi(s)$ has the desired representation form (3.4.4) in the conclusion [I] of the theorem. Moreover, in order to get the conclusion [II] of the theorem, substitute $t$ by $\phi(s)$, which was constructed by (3.4.4) in the conclusion [I], and then it can be easily shown by Lemma 3.4.1 that two irreducible pairs $(y(t), z(t))$ and $\left(y(\phi(t)), z(\phi(t))=\left(y(s), s^{\alpha_{1}}\right)\right.$ are analytically equivalent parametrizations in the conclusion [II]. Thus, this completes the proof of the theorem.

4. The definition of the Puiseux pairs(the multiplicity and Puiseux exponents) and an algorithm for finding the standard Puiseux expansion topologically equivalent to irreducible parametrizations. In this section, first we will rewrite the statement about the definition of the Puiseux pairs in an elementary way, by the same way as we have seen in Definition 1.1. Next, we will classify topologically irreducible parametrizations in terms of the standard Puiseux expansion, using the consequences of Theorem 3.4 in the previous section.

Let $V(f)=\{(y, z): f(y, z)=0\}$ and $V(g)=\{(y, z): g(y, z)=0\}$ be germs of analytic varieties at the origin in $\mathbb{C}^{2}$ where analytic functions $f$ and $g$ at the origin may be assumed to have irreducible parametrizations, respectively. As we have done in Definition 2.8, for simplicity of notations, if $f$ and $g$ have the same topological type of singularity at the origin, we denote this relation by $f \sim g$ or $V(f) \sim V(g)$. Otherwise, we write $f \nsim g$ or $V(f) \nsim V(g)$. Also, if $f$ and $g$ have the same analytic type of singularity at the origin, then we write $f \approx g$. Otherwise, we write $f \not z g$. 
In more detail, for the topological classification of such singularities, it is enough to solve the problem, which is described as follows:

Problem I*: Let the parametrization of an irreducible plane curve $C$ be given by

$$
y=t^{n} \quad \text { and } \quad z=c_{1} t^{k_{1}}+c_{2} t^{k_{2}}+\cdots
$$

where $1<n, 1<k_{1}<k_{2}<\cdots$, and the $c_{i}$ are nonzero complex numbers. Whether or not $n$ is greater than $k_{1}$, find the method how to compute the standard Puiseux expansion(or the Puiseux pairs), which is topologically equivalent to the parametrization of the above curve $C$.

In preparation for the solution of Problem I*, in order to avoid the complexity of the terminology in this section, first of all, we are going to represent the statement of the well-known theorem with the definition of the Puiseux pairs.

DEFINITION 4.1. Let the parametrization for arbitrary irreducible plane curve $C$ be defined by

$$
y(t)=t^{n}, \quad z(t)=c_{1} t^{k_{1}}+c_{2} t^{k_{2}}+\cdots=c_{1} t^{k_{1}}(1+H(t)),
$$

where $1<n, 1<k_{1}<k_{2}<\cdots$, and the $c_{i}$ are nonzero complex numbers and $H(t)$ is just the substitution.

Moreover, note that the curve $C$ is irreducible in $\mathbb{C}\{y, z\} \Longleftrightarrow n \geq \operatorname{gcd}\left(n, k_{1}\right) \geq$ $\operatorname{gcd}\left(n, k_{1}, k_{2}\right) \geq \cdots \geq \operatorname{gcd}\left(n, k_{1}, k_{2}, \ldots\right)=1$.

Now, consider two cases, respectively.

Case[I] Let $n \leq k_{1}$. Then, the parametrization for the curve $C$ of (4.1.1) is called the Puisuex expansion.

Case[II] Let $n>k_{1}$. Then, the parametrization for the curve $C$ of (4.1.1) is not called the Puisuex expansion.

Case[I] Assume that $n \leq k_{1}$. Now, we can define the sequence $\left\{\gamma_{1}, \gamma_{2}, \ldots, \gamma_{p}\right\}$ from the set $\left\{k_{i}: i=1,2, \ldots\right\}$, consisting of the exponents of the above parameter $t$, as follows: Note that $n$ is the multiplicity of the curve $C$ at the origin.

$(*) \gamma_{1}$ is the smallest positive integer among the exponents $k_{i}$ such that $n>\operatorname{gcd}\left(n, \gamma_{1}\right)$; $\gamma_{2}$ is the smallest positive integer among the exponents $k_{i}$ such that $n>\operatorname{gcd}\left(n, \gamma_{1}\right)>$ $\operatorname{gcd}\left(n, \gamma_{1}, k_{i}\right) ; \gamma_{3}$ is the smallest positive integer among the exponents $k_{i}$ such that $n>\operatorname{gcd}\left(n, \gamma_{1}\right)>\operatorname{gcd}\left(n, \gamma_{1}, \gamma_{2}\right)>\operatorname{gcd}\left(n, \gamma_{1}, \gamma_{2}, k_{i}\right) ; \ldots ; \gamma_{p}$ is the smallest positive integer among the exponents $k_{i}$ such that $n>\operatorname{gcd}\left(n, \gamma_{1}\right)>\operatorname{gcd}\left(n, \gamma_{1}, \gamma_{2}\right)>$ $\operatorname{gcd}\left(n, \gamma_{1}, \gamma_{2}, \gamma_{3}\right)>\cdots>\operatorname{gcd}\left(n, \gamma_{1}, \gamma_{2}, \ldots, \gamma_{p}\right)=1$.

(1) By the uniqueness of construction of the set $\left\{\gamma_{i}: 1 \leq i \leq p\right\}, \gamma_{i}$ is called $i$-th Puiseux exponent in this paper.

(2) By (1), let $\mathrm{S}$ be the set defined by $\left\{n, \gamma_{1}, \gamma_{2}, \ldots, \gamma_{p}\right\}$. Whenever the Puiseux expansion for the curve $C$ is given, then the set $S$ is uniquely determined by the curve $C$.

(2a) In this paper, $S$ is called the multiplicity and Puiseux exponents for the curve $C$, that is, a new terminology.

(2b) If necessary, we can construct uniquely the standard Puiseux expansion defined by $y=t^{n}$ and $z=t^{\gamma_{1}}+t^{\gamma_{2}}+\cdots+t^{\gamma_{p}}$ for the curve $C$. 
(3) By (2), let $d_{i}=\operatorname{gcd}\left(n, \gamma_{1}, \ldots, \gamma_{i}\right)$ for $1 \leq i \leq p$, and write $d_{0}=n$ for brevity of notation.

Define $\lambda_{i}$ and $\mu_{i}$ by $\lambda_{i}=\frac{\gamma_{i}}{d_{i}}$ and $\mu_{i}=\frac{d_{i-1}}{d_{i}}$ for $1 \leq i \leq p$, and let $\left(\lambda_{i}, \mu_{i}\right)$ be defined by the Puiseux pair for each $i$.

Then, $\left\{\left(\lambda_{i}, \mu_{i}\right): i=1,2, \ldots, p\right\}$ is called a finite sequence of Puiseux pairs for the curve $C$. If necessary, this sequence will be sometimes called the set of Puiseux pairs for the curve $C$.

(4) By the next remark, it can be shown that there is a one-to-one correspondence between the set of the multiplicity and Puiseux exponents, and the set of Puiseux pairs, that is, (2) and (3) have the same type of definitions arithmetically.

(4a) If the parametrization defined by $(y(t), z(t))$ in (1.0.1) is the Puiseux expansion, then it is said that this Puiseux expansion have either the multiplicity and Puiseux exponents $\left\{n, \gamma_{1}, \gamma_{2}, \ldots, \gamma_{p}\right\}$ or the Puiseux pairs $\left\{\left(\lambda_{i}, \mu_{i}\right): i=1,2, \ldots, p\right\}$ where each $\lambda_{i}$ and $\mu_{i}$ is defined as we have seen in (3).

(5) By (4), throughout this paper, we prefer to choose the terminology in (2) rather than that in (3), if necessary.

Case[II] Assume that $n>k_{1}$. For the convenience of the notation, we may begin without loss of generality that the parametrization of the pair $(y(t), z(t))$ for the curve $C$ of (4.1.1) is written in the following:

$$
y(t)=t^{m}, \quad z(t)=b_{1} t^{\beta_{1}}+b_{2} t^{\beta_{2}}+\cdots, \quad \text { with } m>\beta_{1}
$$

where the $b_{i}$ are nonzero complex numbers, and $m>1$ and $1<\beta_{1}<\beta_{2}<\beta_{3}<\cdots$, and $m \geq \operatorname{gcd}\left(m, \beta_{1}\right) \geq \operatorname{gcd}\left(m, \beta_{1}, \beta_{2}\right) \geq \cdots \geq \operatorname{gcd}\left(n, \beta_{1}, \beta_{2}, \ldots\right)=1$.

By (4.1.2), let $s$ be the new parameter defined by a conformal mapping

$$
s(t)=t\left(b_{1}+\sum_{i \geq 2} b_{i} t^{\beta_{i}-\beta_{1}}\right)^{\frac{1}{\beta_{1}}}
$$

of $t$ at the origin such that $z(t)=(s(t))^{\beta_{1}}$ and $s(0)=0$, and let $t=\phi(s)$ be its inverse.

Then, the Puiseux expansion defined by $y_{1}(s)=y(\phi(s))$ and $z_{1}(s)=z(\phi(s))$, which is equivalent to the parametrization of the pair $(y(t), z(t))$ in $(4.1 .2)$, can be written as follows:

$$
z_{1}(s)=s^{\beta_{1}}, \quad y_{1}(s)=c_{1} s^{\ell_{1}}+c_{2} s^{\ell_{2}}+\cdots, \quad \text { with } \beta_{1}<\ell_{1}
$$

where $1<m=\ell_{1}<\ell_{2}<\cdots$, and $\beta_{1}<\ell_{1}$, and the $c_{i}$ are nonzero complex numbers.

Therefore, if $m=\ell_{1}$ is greater than $\beta_{1}$, then by using Theorem 3.4 in this paper, first we will find the inverse $t=\phi(s)$ of a conformal mapping $s=s(t)$ in (4.1.2), which gives an algorithm for the construction of the Puiseux expansion in (4.1.4), that is, an equivalent parametrization for the above curve $C$. Next, applying the new terminology to this case, by the same way as we have used in Case[I] of this definition, we can naturally generalize the definition of the following words for this curve $C$ of (4.1.4) in Case[II], respectively:

The multiplicity and Puiseux exponents, the standard Puiseux expansion, a finite sequence of the Puiseux pairs. 
REMARK 4.1.1. If either the multiplicity and Puiseux exponents, or the standard Puiseux expansion for the curve are given, then it is clear that a finite sequence of the Puiseux pairs is uniquely determined. Conversely, if a finite sequence of the Puiseux pairs is given, then we show that the construction of the multiplicity and the Puiseux exponents are trivial, which can be easily proved from the following computations:

For the proof, follow the same notation as we have seen in both (2) and (3) of Case[I] of Definition 4.1. Since $\left(\lambda_{p}, \mu_{p}\right)$ is given and $d_{p}=1$ from the assumption, then we can compute $\gamma_{p}$ and $d_{p-1}$ because $\lambda_{p}=\gamma_{p}$ and $\mu_{p}=d_{p-1}$. If $p=1$, then $\gamma_{p}$ and $d_{p-1}=n$ were already computed, and so the proof is done. Let $p>1$. Next, since $\left(\lambda_{p-1}, \mu_{p-1}\right)$ is given by assumption, and $\gamma_{p}$ and $d_{p-1}$ were already computed, then we can compute $\gamma_{p-1}$ and $d_{p-2}$ because $\lambda_{p-1}=\frac{\gamma_{p-1}}{d_{p-1}}$ and $\mu_{p-1}=\frac{d_{p-2}}{d_{p-1}}$. If $p=2$, then $\gamma_{p}, \gamma_{p-1}$, and $d_{p-2}=n$ were already computed, and so the proof is done. Let $p>2$. Thus, following the induction method on the positive integer $p$, the proof can be easily done with $d_{0}=n$.

Solution for Problem I*: In order to solve the problem rigorously, it is enough to consider two cases, respectively:

Case(i): Let $n \leq k_{1}$.

Case(ii): Let $n>k_{1}$.

Case(i): If $n \leq k_{1}$, then recall that the above parametrization of the curve $C$ is called the Puiseux expansion for the above curve.

For this case, we may begin with the following well-known theorem, without proof:

The well-known theorem(Theorem B) As far as arbitrary Puiseux parametrizations of irreducible plane curve singularities are concerned, any two irreducible plane curve singularities have the same topological types if and only if they have the same Puiseux pairs.

Therefore, using Theorem $2.9([\mathrm{Br}],[\mathrm{Bu}], \mathrm{Z1}])$ and Lemma 3.3 , it is very interesting that Theorem B will be represented more concretely by the following theorem(Theorem 4.2).

Case(ii): If $n>k_{1}$, first apply Lemma 3.3 and Theorem 3.4 with the inverse $t=\phi(s)$ of a conformal mapping $s=s(t)$ in (3.1.2) of Definition 3.1, to this case, and then it is easy to compute the Puiseux expansion topologically equivalent to the curve $C$. We will see later by Definition 4.4 and Theorem 4.5 that to find such an expansion is still trivial, using the consequences of Case(i).

Now, if using Theorem 2.9([Br],[Bu],Z1]) and Lemma 3.3, then we can restate more concretely the above well-known theorem without any other proof, as follows:

THEOREM 4.2 (THE WELL-KNOWN THEOREM). Let the parametrization of any irreducible plane curve $V(f)$ be given by

$$
y=t^{n} \quad \text { and } \quad z=c_{1} t^{k_{1}}+c_{2} t^{k_{2}}+\cdots,
$$

where $1<n, 1<k_{1}<k_{2}<\cdots$, and the $c_{i}$ are nonzero complex numbers.

By Lemma 3.3, the irreducible curve $V(f)$ of (4.2.1) can be easily rearranged in 
the form

$$
\begin{aligned}
y= & t^{n} \\
z= & a_{1} t^{\alpha_{1}}\left(1+D_{1}(t)\right)+a_{2} t^{\alpha_{2}}\left(1+D_{2}(t)\right)+\cdots \\
& +a_{r} t^{\alpha_{r}}\left(1+D_{r}(t)\right)+a_{r+1} t^{\alpha_{r+1}}\left(1+D_{r+1}(t)\right) \\
= & a_{1} t^{\alpha_{1}}(1+H(t)),
\end{aligned}
$$

where

(i) $2 \leq n$ and $2 \leq \alpha_{1}=k_{1}<\alpha_{2}<\cdots<\alpha_{r+1}$,

(ii) $n \geq d_{1}>d_{2}>\cdots>d_{r+1}=1$ with $\operatorname{gcd}\left(n, \alpha_{1}, \ldots, \alpha_{i}\right)=d_{i}$ for $1 \leq i \leq r+1$,

(iii) $p_{1}, p_{2}, \ldots, p_{r}$ are nonnegative integers such that $p_{i} d_{i}<\alpha_{i+1}-\alpha_{i}<\left(p_{i}+1\right) d_{i}$ for $1 \leq i \leq r$, $\mathbb{C}\{t\}$

(iv) $\bar{D}_{j}(t)=\sum_{i=1}^{p_{j}} c_{j i} t^{i d_{j}} \in \mathbb{C}[t]$ for $1 \leq j \leq r$ and $D_{r+1}(t)=\sum_{i=1}^{\infty} c_{j+1, i} t^{i} \in$

(v) $1+H(t)=1+D_{1}(t)+t^{\alpha_{2}-\alpha_{1}}\left(c_{20}+D_{2}(t)\right)+\cdots \quad+t^{\alpha_{r}-\alpha_{1}}\left(c_{r 0}+D_{r}(t)\right)+$ $t^{\alpha_{r+1}-\alpha_{1}}\left(c_{r+1,0}+D_{r+1}(t)\right)$ with $c_{i 0}=\frac{a_{i}}{a_{1}}$ for $2 \leq i \leq r+1$,

(vi) the $a_{i}$ are nonzero complex numbers with $a_{1}=c_{1}$ for $i=1,2, \ldots, r+1$, from the coefficients $c_{i}$ of (4.2.1).

Now, for the representation of the statement of the well-known theorem, assume that $n \leq k_{1}$. Then, we have the following conclusion:

(1) If $n>\operatorname{gcd}\left(n, \alpha_{1}\right)=d_{1}$, then $V(f) \sim C_{1}$ where $C_{1}$ is the curve parametrized by the standard Puiseux expansion

$$
C_{1}:=\left\{\begin{array}{l}
y=t^{n} \\
z=t^{\alpha_{1}}+t^{\alpha_{2}}+\cdots+t^{\alpha_{r+1}} .
\end{array}\right.
$$

(2) If $n=\operatorname{gcd}\left(n, \alpha_{1}\right)=d_{1}$, then $V(f) \sim C_{2}$ where $C_{2}$ is the curve parametrized by the standard Puiseux expansion

$$
C_{2}:=\left\{\begin{array}{l}
y=t^{n} \\
z=t^{\alpha_{2}}+t^{\alpha_{3}}+\cdots+t^{\alpha_{r+1}} .
\end{array}\right.
$$

Moreover, the standard Puiseux expansion which is topologically equivalent to the Puiseux expansion of the curve $V(f)$ is uniquely determined.

REMARK 4.2.1. Summarizing (1) and (2) in Theorem 4.2, whether or not $n$ a divisor of $\alpha_{1}$, then $V(f) \sim C^{*}$ where $C^{*}$ is the curve parametrized by the Puiseux expansion

$$
C^{*}:=\left\{\begin{array}{l}
y=t^{n} \\
z=t^{\alpha_{1}}+t^{\alpha_{2}}+t^{\alpha_{3}}+\cdots+t^{\alpha_{r+1}} .
\end{array}\right.
$$

In particular, if $n \leq k_{1}$ and $n$ is a divisor of $k_{1}$, then for a later application, we are going to study the curve $V(f)$ of (4.2.2) by Lemma 4.3 that we can construct a local biholomorphic mapping $\phi$ from $(y, z)=(0,0)$ to $\left(y, z^{\prime}\right)=(0,0)$ and $f \circ \phi$ as follows:

$$
\begin{aligned}
\phi(y, z) & =\left(y, z^{\prime}\right) \quad \text { with } \\
z^{\prime} & =z-a_{1} t^{\alpha_{1}}\left(1+D_{1}(t)\right)=z-g(y) \quad \text { and } \\
V(f) & \approx V(f \circ \phi)
\end{aligned}
$$


where $g(y)$ is holomorphic at the origin and $g(0)=0$.

\section{LEMMA 4.3.}

Assumption Let $f(y, z)$ be analytically irreducible in $\mathbb{C}\{y, z\}$ with isolated singularity at the origin in $\mathbb{C}^{2}$. Assume that the curve $V(f)$ defined by the above analytic function $f$ at the origin has an irreducible parametrization as follows:

$$
V(f):=\left\{\begin{aligned}
y= & t^{n} \\
z= & a_{1} t^{\alpha_{1}}\left(1+D_{1}(t)\right)+a_{2} t^{\alpha_{2}}\left(1+D_{2}(t)\right) \\
& \quad+\cdots+a_{r} t^{\alpha_{r}}\left(1+D_{r}(t)\right)+a_{r+1} t^{\alpha_{r+1}}\left(1+D_{r+1}(t)\right),
\end{aligned}\right.
$$

where

(1a) $2 \leq n<\alpha_{1}<\alpha_{2}<\cdots<\alpha_{r+1}$

(1b) $n \geq \operatorname{gcd}\left(n, \alpha_{1}\right)>\operatorname{gcd}\left(n, \alpha_{1}, \alpha_{2}\right)>\cdots>\operatorname{gcd}\left(n, \alpha_{1}, \alpha_{2}, \ldots, \alpha_{r+1}\right)=1$, and $n$ is a divisor of $\alpha_{1}$,

(1c) the $a_{i}$ are all nonzero numbers for $i=1,2, \ldots, r+1$,

(1d) write $d_{1}=\operatorname{gcd}\left(n, \alpha_{1}\right), d_{2}=\operatorname{gcd}\left(n, \alpha_{1}, \alpha_{2}\right), \ldots, d_{r}=\operatorname{gcd}\left(n, \alpha_{1}, \ldots, \alpha_{r}\right)$, $d_{r+1}=\operatorname{gcd}\left(n, \alpha_{1}, \ldots, \alpha_{r+1}\right)=1$, and then define $p_{1}, p_{2}, \ldots, p_{r}$ to be nonnegative integers such that

$$
\begin{aligned}
p_{1} d_{1} & <\alpha_{2}-\alpha_{1}<\left(p_{1}+1\right) d_{1}, \\
p_{2} d_{2} & <\alpha_{3}-\alpha_{2}<\left(p_{2}+1\right) d_{2}, \\
& \ldots \ldots \\
& \ldots \\
p_{r-1} & <\alpha_{r}-\alpha_{1}<\left(p_{r-1}+1\right) d_{r-1}, \\
p_{r} d_{r} & <\alpha_{r+1}-\alpha_{r}<\left(p_{r}+1\right) d_{r}
\end{aligned}
$$

(1e) for $i=1,2, \ldots, r+1$, define $D_{i}(t)$ by

$$
\begin{aligned}
D_{1}(t) & =\sum_{i=1}^{p_{1}} a_{1 i} t^{i d_{1}} \in \mathbb{C}[t], \\
D_{2}(t) & =\sum_{i=1}^{p_{2}} a_{2 i} t^{i d_{2}} \in \mathbb{C}[t], \\
& \cdots \\
D_{r}(t) & =\sum_{i=1}^{p_{r}} a_{r i} t^{i d_{r}} \in \mathbb{C}[t], \\
D_{r+1}(t) & =\sum_{i=1}^{\infty} a_{r+1, i} t^{i} \in \mathbb{C}\{t\},
\end{aligned}
$$

such that all $a_{j, i(j)}$ are complex numbers with $1 \leq j \leq r+1$ and $1 \leq i(j) \leq p_{j}$, some of which may be zero. Note that $p_{r+1}$ may be infinite.

Conclusion Let $n$ be a divisor of $\alpha_{1}$, and then write $\alpha_{1}=n k$ for some positive integer $k$. Define $z^{\prime}=z(t)-a_{1} t^{\alpha_{1}}\left(1+D_{1}(t)\right)$. Then, we have the followings:

(i) Then, $z^{\prime}=z-a_{1} y^{k}\left(1+\sum_{i=1}^{p_{1}} a_{1 i} y^{i}\right)$ can be viewed as an element in $\mathbb{C}\{y, z\}$, and so $\phi(y, z)=\left(y, z^{\prime}\right)$ with $\phi(0,0)=(0,0)$ is locally a biholomorphic mapping at $(y, z)=(0,0)$. 
(ii) Let $C^{\prime}$ be the irreducible curve parametrized by $y=t^{n}$ and $z^{\prime}=z^{\prime}(t)$. Then, the curve $C^{\prime}$ and $V(f)$ have the same analytic type of singularity at the origin. In other words, if $n$ is a divisor of $\alpha_{1}$, then the singularity of $V(f)$ is analytically invariant at the origin, whether or not $a_{1} \neq 0$.

Proof of Lemma 4.3. Assume that $n$ is a divisor of $\alpha_{1}$, and $\alpha_{1}=n k$ for some integer $k$. Then, $d_{1}=\operatorname{gcd}\left(n, \alpha_{1}\right)=n$. Observe the followings:

$$
\begin{aligned}
z^{\prime} & =z-a_{1} t^{\alpha_{1}}\left(1+D_{1}(t)\right) \\
& =a_{2} t^{\alpha_{2}}\left(1+D_{2}(t)\right)+\cdots+a_{r} t^{\alpha_{r}}\left(1+D_{r}(t)\right)+a_{r+1} t^{\alpha_{r+1}}\left(1+D_{r+1}(t)\right),
\end{aligned}
$$

where $D_{1}(t)=\sum_{i=1}^{p_{1}} a_{1 i} t^{i d_{1}}$ is in $\mathbb{C}[t]$. So, using $y=t^{n}$ and $n=d_{1}, D_{1}(t)=$ $\sum_{i=1}^{p_{1}} a_{1 i} y^{i}$, and then $z^{\prime}=z-a_{1} y^{k}\left(1+\sum_{i=1}^{p_{1}} a_{1 i} y^{i}\right)$. Now, define a local holomorphic mapping $\phi$ from the origin to the origin as follows:

$$
\phi(y, z)=\left(y, z^{\prime}\right)
$$

It is easy to check that $\phi$ is biholomorphic at the origin with $\phi(0,0)=(0,0)$, and then the proofs of (i) and (ii) can be easily shown.

Now, we are going to generalize Definition 4.1 by the following.

DeFinition 4.4. Let $f(y, z)$ be analytically irreducible in $\mathbb{C}\{y, z\}$ with isolated singularity at the origin in $\mathbb{C}^{2}$. By Lemma 3.3, we may assume without loss of generality that the curve $V(f)$ defined by the above $f$ at the origin has an irreducible parametrization as follows:

$$
V(f):=\left\{\begin{aligned}
y= & t^{n} \\
z= & a_{1} t^{\alpha_{1}}\left(1+D_{1}(t)\right)+a_{2} t^{\alpha_{2}}\left(1+D_{2}(t)\right) \\
& \quad+\cdots+a_{r} t^{\alpha_{r}}\left(1+D_{r}(t)\right)+a_{r+1} t^{\alpha_{r+1}}\left(1+D_{r+1}(t)\right),
\end{aligned}\right.
$$

where

(1a) $2 \leq n$ and $2 \leq \alpha_{1}<\alpha_{2}<\cdots<\alpha_{r+1}$,

(1b) $n \geq \operatorname{gcd}\left(n, \alpha_{1}\right)>\operatorname{gcd}\left(n, \alpha_{1}, \alpha_{2}\right)>\cdots>\operatorname{gcd}\left(n, \alpha_{1}, \alpha_{2}, \ldots, \alpha_{r+1}\right)=1$,

(1c) the $a_{i}$ are all nonzero numbers for $i=1,2, \ldots, r+1$,

(1d) write $d_{1}=\operatorname{gcd}\left(n, \alpha_{1}\right), d_{2}=\operatorname{gcd}\left(n, \alpha_{1}, \alpha_{2}\right), \ldots, d_{r}=\operatorname{gcd}\left(n, \alpha_{1}, \ldots, \alpha_{r}\right)$, $d_{r+1}=\operatorname{gcd}\left(n, \alpha_{1}, \ldots, \alpha_{r+1}\right)=1$, and then define $p_{1}, p_{2}, \ldots, p_{r}$ to be nonnegative integers such that

$$
\begin{aligned}
p_{1} d_{1}<\alpha_{2}-\alpha_{1}<\left(p_{1}+1\right) d_{1}, \\
p_{2} d_{2}<\alpha_{3}-\alpha_{2}<\left(p_{2}+1\right) d_{2}, \\
\quad \ldots \ldots \\
\quad \ldots \\
p_{r-1}<\alpha_{r}-\alpha_{1}<\left(p_{r-1}+1\right) d_{r-1}, \\
p_{r} d_{r}<\alpha_{r+1}-\alpha_{r}<\left(p_{r}+1\right) d_{r},
\end{aligned}
$$


(1e) for $i=1,2, \ldots, r+1$, define $D_{i}(t)$ by

$$
\begin{aligned}
D_{1}(t) & =\sum_{i=1}^{p_{1}} a_{1 i} t^{i d_{1}} \in \mathbb{C}[t], \\
D_{2}(t) & =\sum_{i=1}^{p_{2}} a_{2 i} t^{i d_{2}} \in \mathbb{C}[t], \\
& \ldots \\
D_{r}(t) & =\sum_{i=1}^{p_{r}} a_{r i} t^{i d_{r}} \in \mathbb{C}[t], \\
D_{r+1}(t) & =\sum_{i=1}^{\infty} a_{r+1, i} t^{i} \in \mathbb{C}\{t\},
\end{aligned}
$$

such that all $a_{j, i(j)}$ are complex numbers with $1 \leq j \leq r+1$ and $1 \leq i(j) \leq p_{j}$, some of which may be zero. Note that $p_{r+1}$ may'be infinite.

Then, the multiplicity and Puiseux exponents for the curve $V(f)$ are defined as follows:

(A) If $n \leq \alpha_{1}$ and $n$ is not a divisor of $\alpha_{1}$, then note that the parametrization defined by $y=t^{n}$ and $z=t^{\alpha_{1}}+t^{\alpha_{2}}+\cdots+t^{\alpha_{r+1}}$ is called the standard Puiseux expansion. Then, it is said that the set $\left\{n, \alpha_{1}, \alpha_{2}, \ldots, \alpha_{r+1}\right\}$ is a finite sequence of the multiplicity and Puiseux exponents for the Puiseux expansion of $V(f)$.

(B) If $n \leq \alpha_{1}$ and $n$ is a divisor of $\alpha_{1}$, then note that the parametrization defined by $y=t^{n}$ and $z=t^{\alpha_{2}}+t^{\alpha_{3}}+\cdots+t^{\alpha_{r+1}}$ is called the standard Puiseux expansion. Then, it is said that the set $\left\{n, \alpha_{2}, \alpha_{3}, \ldots, \alpha_{r+1}\right\}$ is a finite sequence of the multiplicity and Puiseux exponents for the Puiseux expansion of $V(f)$.

In case $n>\alpha_{1}$, using the equation of (3.4.6) in the conclusion of Theorem 3.4, we can compute the Puiseux expansion which is equivalent to the parametrization of $V(f)$, as follows:

$$
V(f): \approx\left\{\begin{aligned}
z= & s^{\alpha_{1}} \\
y= & c_{1}^{-\frac{n}{\alpha_{1}}} s^{n}\left\{\left(1+Q_{1}^{*}(s)\right)+s^{\alpha_{2}-\alpha_{1}}\left(b_{20}+Q_{2}^{*}(s)\right)\right. \\
& \left.+s^{\alpha_{3}-\alpha_{1}}\left(b_{30}+Q_{3}^{*}(s)\right)+\cdots+s^{\alpha_{r+1}-\alpha_{1}}\left(b_{r+1,0}+Q_{r+1}^{*}(s)\right)\right\} \\
= & c_{1}^{-\frac{n}{\alpha_{1}}} s^{n}\{1+L(s)\},
\end{aligned}\right.
$$

where

(i) $\operatorname{gcd}\left(n, \alpha_{1}, \alpha_{2}-\alpha_{1}, \ldots, \alpha_{i}-\alpha_{1}\right)=\operatorname{gcd}\left(n, \alpha_{1}, \alpha_{2}, \ldots, \alpha_{i}\right)=d_{i}$ for $1 \leq i \leq r+1$,

(ii) $Q_{j}^{*}(s)=\sum_{i=1}^{p_{j}} b_{j i} s^{i d_{j}} \in \mathbb{C}[s]$ for $1 \leq j \leq r$ and $Q_{r+1}^{*}(s)=\sum_{i=1}^{\infty} b_{r+1, i} s^{i} \in$ $\mathbb{C}\{s\}$,

(iii) all the $b_{j, i(j)}$ are complex numbers with $1 \leq j \leq r+1$ and $1 \leq i(j) \leq p_{j}$, noting that $p_{r+1}$ may be infinite,

(iv*) the $b_{j, 0}$ are all nonzero complex numbers for $2 \leq j \leq r+1$, noting that $b_{j, 0}=n B_{j, 0}$ for $2 \leq j \leq r+1$,

(v) $L(s)$ is just the substitution.

By the same method as we have done in two cases (A) and (B), then it is enough 
to consider the following cases:

(C) If $n>\alpha_{1}$ and $\alpha_{1}>\operatorname{gcd}\left(n, \alpha_{1}\right)$, then it is said that $y=t^{\alpha_{1}}$ and $z=t^{n}+$ $t^{n+\alpha_{2}-\alpha_{1}}+t^{n+\alpha_{3}-\alpha_{1}}+\cdots+t^{n+\alpha_{r+1}-\alpha_{1}}$ is the standard Puiseux expansion. Then, it is said that the set $\left\{\alpha_{1}, n, n+\alpha_{2}-\alpha_{1}, n+\alpha_{3}-\alpha_{1}, \ldots, n+\alpha_{r+1}-\alpha_{1}\right\}$ is a finite sequence of the multiplicity and Puiseux exponents for the curve $V(f)$.

(D) If $n>\alpha_{1}$ and $\alpha_{1}$ is a divisor of $n$, then it is said that $y=t^{\alpha_{1}}$ and $z=$ $t^{n+\alpha_{2}-\alpha_{1}}+t^{n+\alpha_{3}-\alpha_{1}}+\cdots+t^{n+\alpha_{r+1}-\alpha_{1}}$ is the standard Puiseux expansion. Then, it is said that the set $\left\{\alpha_{1}, n+\alpha_{2}-\alpha_{1}, n+\alpha_{3}-\alpha_{1}, \ldots, n+\alpha_{r+1}-\alpha_{1}\right\}$ is a finite sequence of the multiplicity and Puiseux exponents for the curve $V(f)$.

REMARK 4.4.1. We will prove by the next theorem(Theorem 4.5) that for any parametrization of a given irreducible plane curve singularity $V(f)$, which has the same type as in Definition 4.4, the multiplicity and Puiseux exponents(equivalently, the Puiseux pairs) for $V(f)$ are topological invariant. In other words, it will be proved by Theorem 4.5 that Definition 4.4 is topologically well-defined.

TheOrem 4.5 (An Algorithm For Finding the STANDARd PuiseuX EXPANSIONS TOPOLOGICALLY EQUIVALENT TO GIVEN IRREDUCIBLE PARAMETRIZATIONS).

Assumption Let $f(y, z), g(y, z)$ and $h(y, z)$ be analytically irreducible in $\mathbb{C}\{y, z\}$ with isolated singularity at the origin in $\mathbb{C}^{2}$. Assume that three curves $V(f), V(g)$ and $V(h)$ defined by the above analytic functions $f, g$ and $h$ at the origin have irreducible parametrizations, respectively as follows:

(1) Let the parametrization of $V(f)$ be the Puiseux expansion with the multiplicity and Puiseux exponents defined by

$$
V(f):=\left\{\begin{aligned}
y= & t^{n} \\
z= & a_{1} t^{\alpha_{1}}\left(1+D_{1}(t)\right)+a_{2} t^{\alpha_{2}}\left(1+D_{2}(t)\right) \\
& \quad+\cdots+a_{r} t^{\alpha_{r}}\left(1+D_{r}(t)\right)+a_{r+1} t^{\alpha_{r+1}}\left(1+D_{r+1}(t)\right),
\end{aligned}\right.
$$

where

(1a) $2 \leq n<\alpha_{1}<\alpha_{2}<\cdots<\alpha_{r+1}$

(1b*) $n>\operatorname{gcd}\left(n, \alpha_{1}\right)>\cdots>\operatorname{gcd}\left(n, \alpha_{1}, \ldots, \alpha_{r+1}\right)=1$,

(1c) the $a_{i}$ are all nonzero numbers for $i=1,2, \ldots, r+1$,

(1d) write $d_{1}=\operatorname{gcd}\left(n, \alpha_{1}\right), d_{2}=\operatorname{gcd}\left(n, \alpha_{1}, \alpha_{2}\right), \ldots, d_{r}=\operatorname{gcd}\left(n, \alpha_{1}, \ldots, \alpha_{r}\right)$, $d_{r+1}=\operatorname{gcd}\left(n, \alpha_{1}, \ldots, \alpha_{r+1}\right)=1$, and then define $p_{1}, p_{2}, \ldots, p_{r}$ to be nonnegative integers such that

$$
\begin{aligned}
p_{1} d_{1} & <\alpha_{2}-\alpha_{1}<\left(p_{1}+1\right) d_{1}, \\
p_{2} d_{2} & <\alpha_{3}-\alpha_{2}<\left(p_{2}+1\right) d_{2}, \\
& \ldots \\
& \ldots \\
p_{r-1} & <\alpha_{r}-\alpha_{r-1}<\left(p_{r-1}+1\right) d_{r-1}, \\
p_{r} d_{r} & <\alpha_{r+1}-\alpha_{r}<\left(p_{r}+1\right) d_{r},
\end{aligned}
$$


(1e) for $i=1,2, \ldots, r+1$, define $D_{i}(t)$ by

$$
\begin{aligned}
D_{1}(t) & =\sum_{i=1}^{p_{1}} a_{1 i} t^{i d_{1}} \in \mathbb{C}[t], \\
D_{2}(t) & =\sum_{i=1}^{p_{2}} a_{2 i} t^{i d_{2}} \in \mathbb{C}[t], \\
& \cdots \\
D_{r}(t) & =\sum_{i=1}^{p_{r}} a_{r i} t^{i d_{r}} \in \mathbb{C}[t], \\
D_{r+1}(t) & =\sum_{i=1}^{\infty} a_{r+1, i} t^{i} \in \mathbb{C}\{t\},
\end{aligned}
$$

such that all $a_{j, i(j)}$ are complex numbers with $1 \leq j \leq r+1$ and $1 \leq i(j) \leq p_{j}$, some of which may be zero. Note that $p_{r+1}$ may be infinite.

Remark: In the above condition (ib*) of (4.5.1), if $n \geq \operatorname{gcd}\left(n, \alpha_{1}\right)$ and $n$ is a divisor of $\alpha_{1}$, then by Lemma 4.3 the singularity of $V(f)$ is analytically invariant at the origin, whether or not $a_{1}$ is zero, and so from the beginning we may assume without loss of generality that $n>\operatorname{gcd}\left(n, \alpha_{1}\right)$.

(2) Let the parametrization of $V(g)$ be the Puiseux expansion with the multiplicity and Puiseux exponents defined by

$$
V(g):=\left\{\begin{aligned}
y= & t^{m} \\
z= & b_{1} t^{\beta_{1}}\left(1+L_{1}(t)\right)+b_{2} t^{\beta_{2}}\left(1+L_{2}(t)\right) \\
& \quad+\cdots+b_{u} t^{\beta_{u}}\left(1+L_{u}(t)\right)+b_{u+1} t^{\beta_{u+1}}\left(1+L_{u+1}(t)\right)
\end{aligned}\right.
$$

where

(2a) $2 \leq m<\beta_{1}<\beta_{2}<\cdots<\beta_{u+1}$,

(2b) $m>\operatorname{gcd}\left(m, \beta_{1}\right)>\cdots>\operatorname{gcd}\left(m, \beta_{1}, \cdots, \beta_{u+1}\right)=1$,

(2c) the $b_{i}$ are all nonzero numbers for $i=1,2, \ldots, u+1$,

(2d) write $e_{1}=\operatorname{gcd}\left(m, \beta_{1}\right), e_{2}=\operatorname{gcd}\left(m, \beta_{1}, \beta_{2}\right), \ldots, e_{u}=\operatorname{gcd}\left(m, \beta_{1}, \ldots, \beta_{u}\right)$, $e_{u+1}=\operatorname{gcd}\left(m, \beta_{1}, \ldots, \beta_{u+1}\right)=1$, and then define $q_{1}, q_{2}, \ldots, q_{u}$ to be nonnegative integers such that

$$
\begin{aligned}
q_{1} e_{1} & <\beta_{2}-\beta_{1}<\left(q_{1}+1\right) e_{1}, \\
q_{2} e_{2} & <\beta_{3}-\beta_{2}<\left(q_{2}+1\right) e_{2}, \\
& \ldots \\
q_{u-1} e_{u-1} & <\beta_{u}-\beta_{u-1}<\left(q_{u-1}+1\right) e_{u-1}, \\
q_{u} e_{u} & <\beta_{u+1}-\beta_{u}<\left(q_{u}+1\right) e_{u},
\end{aligned}
$$


(2e) for $i=1,2, \ldots, u+1$, define $L_{j}(t)$ by

$$
\begin{aligned}
L_{1}(t) & =\sum_{i=1}^{q_{1}} b_{1 i} t^{i e_{1}} \in \mathbb{C}[t], \\
L_{2}(t) & =\sum_{i=1}^{q_{2}} b_{2 i} t^{i e_{2}} \in \mathbb{C}[t], \\
& \ldots \\
L_{u}(t) & =\sum_{i=1}^{q_{u}} b_{u i} t^{i e_{u}} \in \mathbb{C}[t], \\
L_{u+1}(t) & =\sum_{i=1}^{\infty} b_{u+1, i} t^{i} \in \mathbb{C}\{t\},
\end{aligned}
$$

such that all $b_{j, i(j)}$ are complex numbers with $1 \leq j \leq u+1$ and $1 \leq i(j) \leq q_{j}$, some of which may be zero. Note that $q_{u+1}$ may be infinite.

(3) Let the parametrization of $V(h)$ be defined by

$$
V(h):=\left\{\begin{aligned}
y= & c_{1} t^{l_{1}}\left(1+R_{1}(t)\right)+c_{2} t^{l_{2}}\left(1+R_{2}(t)\right) \\
& +\cdots+c_{v} t^{l_{v}}\left(1+R_{v}(t)\right)+c_{v+1} t^{l_{v+1}}\left(1+R_{v+1}(t)\right) \\
z=t^{\gamma} &
\end{aligned}\right.
$$

where

(3a) $2 \leq l_{1}<\gamma$ and $l_{1}<l_{2}<\cdots<l_{v+1}$,

(3b) $l_{1} \geq \operatorname{gcd}\left(\gamma, l_{1}\right)>\operatorname{gcd}\left(\gamma, l_{1}, l_{2}\right)>\cdots>\operatorname{gcd}\left(\gamma, l_{1}, l_{2}, \ldots, l_{v+1}\right)=1$,

(3c) the $c_{i}$ are all nonzero numbers for $i=1,2, \ldots, v+1$,

(3d) write $\tau_{1}=\operatorname{gcd}\left(\gamma, l_{1}\right), \tau_{2}=\operatorname{gcd}\left(\gamma, l_{1}, l_{2}\right), \ldots, \tau_{v}=\operatorname{gcd}\left(\gamma, l_{1}, l_{2}, \ldots, l_{v}\right)$, $\tau_{v+1}=\operatorname{gcd}\left(\gamma, l_{1}, l_{2}, \ldots, l_{v+1}\right)=1$, and define $\varepsilon_{1}, \varepsilon_{2}, \ldots, \varepsilon_{v}$ to be nonnegative integers such that

$$
\begin{aligned}
\varepsilon_{1} \tau_{1} & <l_{2}-l_{1}<\left(\varepsilon_{1}+1\right) \tau_{1}, \\
\varepsilon_{2} \tau_{2} & <l_{3}-l_{2}<\left(\varepsilon_{2}+1\right) \tau_{2}, \\
& \ldots \\
\varepsilon_{v-1} \tau_{v-1} & <l_{v}-l_{v-1}<\left(\varepsilon_{v-1}+1\right) \tau_{v-1}, \\
\varepsilon_{v} \tau_{v} & <l_{v+1}-l_{v}<\left(\varepsilon_{v}+1\right) \tau_{v},
\end{aligned}
$$


(3e) for $i=1,2, \ldots, v+1$, define $R_{i}(t)$ by

$$
\begin{aligned}
R_{1}(t) & =\sum_{i=1}^{\varepsilon_{1}} c_{1 i} t^{i \tau_{1}} \in \mathbb{C}[t], \\
R_{2}(t) & =\sum_{i=1}^{\varepsilon_{2}} c_{2 i} t^{i \tau_{2}} \in \mathbb{C}[t], \\
& \cdots \\
R_{v}(t) & =\sum_{i=1}^{\varepsilon_{v}} c_{v i} t^{i \tau_{v}} \in \mathbb{C}[t], \\
R_{v+1}(t) & =\sum_{i=1}^{\infty} c_{v+1, i} t^{i} \in \mathbb{C}\{t\}
\end{aligned}
$$

such that all $c_{j, i(j)}$ are complex numbers with $1 \leq j \leq v+1$ and $1 \leq i(j) \leq \varepsilon_{j}$, some of which may be zero. Note that $\varepsilon_{v+1}$ may be infinite, and also that $\mathbb{C}[t]$ is the ring of polynomials in $t$ and $\mathbb{C}\{t\}$ is the ring of convergent power series at $t=0$.

Conclusion We get the followings:

(I) Note that $n>\operatorname{gcd}\left(n, \alpha_{1}\right)$ and $m>\operatorname{gcd}\left(m, \beta_{1}\right)$.

$$
V(f) \sim V(g)
$$

$\Longleftrightarrow$ the multiplicity and Puiseux exponents are the same, by Definition 4.1, that is, $n=m, r+1=u+1$, and $\alpha_{i}=\beta_{i} \quad$ for all $i=1,2, \ldots, r+1$,

$\Longleftrightarrow$ the Puiseux pairs for both $V(f)$ and $V(g)$ are the same.

For example, $V(f)$ is topologically equivalent to the curve defined by $y=t^{n}$ and $z=t^{\alpha_{1}}+t^{\alpha_{2}}+\cdots+t^{\alpha_{r+1}}$, which is the standard expansion of $V(f)$ by Definition 4.1.

(II) Let $\gamma>\ell_{1}$. Then, there are two cases:

(IIa) $l_{1}>\operatorname{gcd}\left(\gamma, l_{1}\right)$ and (IIb) $l_{1}$ is a divisor of $\gamma$.

(IIa) Let $l_{1}>\operatorname{gcd}\left(\gamma, l_{1}\right)$.

Then $V(h) \sim C_{1}$ where $C_{1}$ is the curve parametrized by the standard Puiseux expansion

$$
C_{1}:=\left\{\begin{array}{l}
y=t^{\ell_{1}} \\
z=t^{\gamma}+t^{\gamma+\ell_{2}-\ell_{1}}+\cdots+t^{\gamma+\ell_{r+1}-\ell_{1}} .
\end{array}\right.
$$

So, we get the following:

$$
\begin{aligned}
& V(f) \sim V(h) \\
\Longleftrightarrow & n=l_{1}, \alpha_{1}=\gamma, r+1=v+1 \text { and } \alpha_{i}=\gamma+l_{i}-l_{1} \quad \text { for } \quad 1 \leq i \leq r+1,
\end{aligned}
$$

which is equivalent to the fact that the Puiseux pairs for both $V(f)$ and $V(h)$ are the same in the sense of Definition 4.4 . 
For example, $V(h)$ can be topologically equivalent to the curve defined by any of the following two irreducible parametrizations (i) and (ii):

(i) $y=t^{l_{1}}+t^{l_{2}}+\cdots+t^{l_{v+1}}$ and $z=t^{\gamma}$.

(ii) $y=t^{l_{1}}$ and $z=t^{\gamma}+t^{\gamma+l_{2}-l_{1}}+t^{\gamma+l_{3}-l_{1}}+\cdots+t^{\gamma+l_{v+1}-l_{1}}$,

noting that the above (ii) is the standard Puiseux expansion for $V(h)$.

(IIb) Note that $\gamma>l_{1}$. Let $l_{1}=\operatorname{gcd}\left(\gamma, l_{1}\right)$, that is, $l_{1}$ is a divisor of $\gamma$.

Then $V(h) \sim C_{2}$ where $C_{2}$ is the curve parametrized by the standard Puiseux expansion

$$
C_{2}:=\left\{\begin{array}{l}
y=t^{\ell_{1}} \\
z=t^{\gamma+\ell_{2}-\ell_{1}}+\cdots+t^{\gamma+\ell_{r+1}-\ell_{1}} .
\end{array}\right.
$$

So, we get the following:

$$
\begin{aligned}
& V(f) \sim V(h) \\
\Longleftrightarrow & n=l_{1}, \alpha_{1}=\gamma+l_{2}-l_{1}, r+1=v \text { and } \alpha_{i}=\gamma+l_{i+1}-l_{1} \text { for } 2 \leq i \leq r+1,
\end{aligned}
$$

which is equivalent to the fact that the Puiseux pairs for both $V(f)$ and $V(h)$ are the same in the sense of Definition 4.4 .

For example, $V(h)$ can be topologically equivalent to the curve defined by any of the following two irreducible parametrization (i) and (ii):

(i) $y=t^{l_{1}}+t^{l_{2}}+\cdots+t^{l_{v+1}}$ and $z=t^{\gamma}$.

(ii) $y=t^{l_{1}}$ and $z=\varepsilon t^{\gamma}+t^{\gamma+l_{2}-l_{1}}+t^{\gamma+l_{3}-l_{1}}+\cdots+t^{\gamma+l_{v+1}-l_{1}}$ for any number $\varepsilon$,

noting that if $\varepsilon$ is a zero then the above (ii) is the standard Puiseux expansion for $V(h)$.

Proof of Theorem 4.5. We prove (I) and (II), respectively.

(I) The proof just follows from Lemma 3.3, Theorem 4.2, Theorem 2.9 and Definition 4.4.

(II) Since $\gamma>l_{1}$, then in order to get an equivalent parametrization for the curve $V(h)$ by using the inverse mapping theorem of one complex variable, apply Theorem 3.4 to $V(h)$. After then, by Lemma 3.3 and using the equation of (3.4.6) in the conclusion of Theorem 3.4, and also by Lemma 4.3, Definition 4.4 and the above case (I) of this theorem, there is nothing to prove.

5. Equivalence of the Puiseux expansions with the same multiplicity and Puiseux exponents and the multiplicity sequences for irreducible parametrizations. The second aim in this paper is to prove the following theorem (Theorem $A$ ) in this section in an elementary way, without using the well-known theorem (Theorem $B$ ):

ThEOREM A (THEOREM 5.1): Whenever any two irreducible parametrizations have the same Puiseux pairs (equivalently, the same multiplicity and Puiseux exponents) by a nonsingular change of the parametrization(in the sense of Definition 1.1 or Definition 4.1), then they have the same multiplicity sequences, and conversely. 
Theorem B: As far as arbitrary Puiseux expansion of irreducible plane curve singularities is concerned, any two irreducible plane curve singularities have the same topological types if and only if they have the same Puiseux pairs.

Rigorously speaking about the definition of the Puiseux pair for the curve $C$ with a given irreducible parametrization again (in the sense of Definition 4.1), we may assume that the parametrization of any irreducible plane curve $C$ is given by $y(t)=t^{n}$ and $z(t)=c_{1} t^{k_{1}}+c_{2} t^{k_{2}}+\cdots=c_{1} t^{k_{1}}(1+H(t))$ where $1<n, 1<k_{1}<k_{2}<\cdots$, and the $c_{i}$ are nonzero complex numbers, and $H(t)$ is just the substitution.

If $n \leq k_{1}$, then the irreducible parametrization of the plane curve $C$ is called the Puiseux expansion, and so the Puiseux pairs for the given Puiseux expansion of the curve $C$ has been well-defined.

If $n>k_{1}$, it is very interesting to define the Puiseux pairs of the Puiseux expansion which is equivalent to the parametrization of the curve $C$, as the Puiseux pairs of $C$, as follows:

Let $s$ be the new parameter defined by a conformal mapping of one complex variable $t$ at the origin such that $s(t)=c_{1}^{\frac{1}{k_{1}}} t(1+H(t))^{\frac{1}{k_{1}}}$ with $z=s^{k_{1}}$ and $s(0)=0$, and let $t=\phi(s)$ be its inverse. Then, the paramtrization with the parameter $s$ defined by the type $\left(y_{1}(s), z_{1}(s)\right)=(y(\phi(s)), z(\phi(s)))$, being equivalent to the parametrization of the type $(y(t), z(t))$, is the Puiseux expansion. It can be written in the form

$$
z_{1}(s)=s^{k_{1}}, \quad y_{1}(s)=b_{1} s^{\ell_{1}}+b_{2} s^{\ell_{2}}+\cdots, \quad \text { with } k_{1}<\ell_{1}
$$

where $1<n=\ell_{1}<\ell_{2}<\cdots$, and $k_{1}<n$, and the $b_{i}$ are nonzero complex numbers. In this case, the Puiseux pairs for the curve $C$ can be defined from the Puiseux expansion parametrized by $y_{1}(s)=y(\phi(s))$ and $z_{1}(s)=z(\phi(s))$.

Thus, with the generalized definition of the Puiseux pairs for the curve $C$ with any irreducible parametrization, we will prove Theorem $A$, without using Theorem $B$.

After then, it is clear by Corollary 5.2 that any two irreducible plane curve singularities have the same topological types if and only if they have the same multiplicity sequences, which can be easily proved by Theorem 5.1 and Theorem 4.5 .

Let $V(f)=\{(y, z): f(y, z)=0\}$ and $V(g)=\{(y, z): g(y, z)=0\}$ be germs of analytic varieties at the origin in $\mathbb{C}^{2}$ where analytic functions $f$ and $g$ at the origin may be assumed to have irreducible parametrizations, respectively. For simplicity of notations, if $V(f)$ and $V(g)$ have the same multiplicity sequences, then we write $V(f) \equiv V(g)$ (multi. seq.). Otherwise, we write $V(f) \not \equiv V(g)$ (multi. seq.).

Theorem 5.1 (Equivalence of the Puiseux EXPANSIONS With the SAme

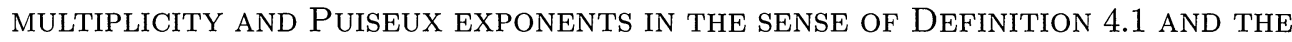
MULTIPLICITY SEQUENCES FOR IRREDUCIBLE PARAMETRIZATIONS).

Assumption Let $f(y, z), g(y, z)$ and $h(y, z)$ be analytically irreducible in $\mathbb{C}\{y, z\}$ with isolated singularity at the origin in $\mathbb{C}^{2}$. Assume that three curves $V(f), V(g)$ and $V(h)$ defined by the above analytic functions $f, g$ and $h$ at the origin have irreducible parametrizations, respectively as follows:

(1) Let the parametrization of $V(f)$ be the Puiseux expansion with the multiplic- 
ities and Puiseux exponents $\left\{n, \alpha_{1}, \alpha_{2}, \ldots, \alpha_{r+1}\right\}$, defined by

$$
V(f):=\left\{\begin{aligned}
y= & t^{n} \\
z= & a_{1} t^{\alpha_{1}}\left(1+D_{1}(t)\right)+a_{2} t^{\alpha_{2}}\left(1+D_{2}(t)\right) \\
& \quad+\cdots+a_{r} t^{\alpha_{r}}\left(1+D_{r}(t)\right)+a_{r+1} t^{\alpha_{r+1}}\left(1+D_{r+1}(t)\right),
\end{aligned}\right.
$$

where

(1a) $2 \leq n<\alpha_{1}<\alpha_{2}<\cdots<\alpha_{r+1}$,

(1b*) $n>\operatorname{gcd}\left(n, \alpha_{1}\right)>\cdots>\operatorname{gcd}\left(n, \alpha_{1}, \ldots, \alpha_{r+1}\right)=1$,

(1c) the $a_{i}$ are all nonzero numbers for $i=1,2, \ldots, r+1$,

(1d) write $d_{1}=\operatorname{gcd}\left(n, \alpha_{1}\right), d_{2}=\operatorname{gcd}\left(n, \alpha_{1}, \alpha_{2}\right), \ldots, d_{r}=\operatorname{gcd}\left(n, \alpha_{1}, \ldots, \alpha_{r}\right)$, $d_{r+1}=\operatorname{gcd}\left(n, \alpha_{1}, \ldots, \alpha_{r+1}\right)=1$, and then define $p_{1}, p_{2}, \ldots, p_{r}$ to be nonnegative integers such that

$$
\begin{aligned}
p_{1} d_{1} & <\alpha_{2}-\alpha_{1}<\left(p_{1}+1\right) d_{1}, \\
p_{2} d_{2} & <\alpha_{3}-\alpha_{2}<\left(p_{2}+1\right) d_{2}, \\
& \ldots \\
p_{r-1} d_{r-1} & <\alpha_{r}-\alpha_{1}<\left(p_{r-1}+1\right) d_{r-1}, \\
p_{r} d_{r} & <\alpha_{r+1}-\alpha_{r}<\left(p_{r}+1\right) d_{r},
\end{aligned}
$$

(1e) for $i=1,2, \ldots, r+1$, define $D_{i}(t)$ by

$$
\begin{aligned}
D_{1}(t) & =\sum_{i=1}^{p_{1}} a_{1 i} t^{i d_{1}} \in \mathbb{C}[t], \\
D_{2}(t) & =\sum_{i=1}^{p_{2}} a_{2 i} t^{i d_{2}} \in \mathbb{C}[t], \\
& \cdots \\
D_{r}(t) & =\sum_{i=1}^{p_{r}} a_{r i} t^{i d_{r}} \in \mathbb{C}[t], \\
D_{r+1}(t) & =\sum_{i=1}^{\infty} a_{r+1, i} t^{i} \in \mathbb{C}\{t\},
\end{aligned}
$$

such that all $a_{j, i(j)}$ are complex numbers with $1 \leq j \leq r+1$ and $1 \leq i(j) \leq p_{j}$, some of which may be zero. Note that $p_{r+1}$ may be infinite.

Remark: In the above condition $\left(1 \mathrm{~b}^{*}\right)$ of (5.1.1), if $n \geq \operatorname{gcd}\left(n, \alpha_{1}\right)$ and $n$ is a divisor of $\alpha_{1}$, then by Lemma 4.3 the singularity of $V(f)$ is analytically invariant at the origin, whether or not $a_{1}$ is zero, and so from the beginning we may assume without loss of generality that $n>\operatorname{gcd}\left(n, \alpha_{1}\right)$ and $a_{1} \neq 0$.

(2) Let the parametrization of $V(g)$ be the Puiseux expansion with the multiplicity and Puiseux exponents $\left\{m, \beta_{1} . \beta_{2}, \ldots, \beta_{u+1}\right\}$, defined by

$$
V(g):=\left\{\begin{aligned}
y= & t^{m} \\
z= & b_{1} t^{\beta_{1}}\left(1+L_{1}(t)\right)+b_{2} t^{\beta_{2}}\left(1+L_{2}(t)\right) \\
& \quad+\cdots+b_{u} t^{\beta_{u}}\left(1+L_{u}(t)\right)+b_{u+1} t^{\beta_{u+1}}\left(1+L_{u+1}(t)\right)
\end{aligned}\right.
$$


where

(2a) $2 \leq m<\beta_{1}<\beta_{2}<\cdots<\beta_{u+1}$,

(2b) $m>\operatorname{gcd}\left(m, \beta_{1}\right)>\cdots>\operatorname{gcd}\left(m, \beta_{1}, \cdots, \beta_{u+1}\right)=1$,

(2c) the $b_{i}$ are all nonzero numbers for $i=1,2, \ldots, u+1$,

(2d) write $e_{1}=\operatorname{gcd}\left(m, \beta_{1}\right), e_{2}=\operatorname{gcd}\left(m, \beta_{1}, \beta_{2}\right), \ldots, e_{u}=\operatorname{gcd}\left(m, \beta_{1}, \ldots, \beta_{u}\right)$, $e_{u+1}=\operatorname{gcd}\left(m, \beta_{1}, \ldots, \beta_{u+1}\right)=1$, and then define $q_{1}, q_{2}, \ldots, q_{u}$ to be nonnegative integers such that

$$
\begin{aligned}
q_{1} e_{1} & <\beta_{2}-\beta_{1}<\left(q_{1}+1\right) e_{1}, \\
q_{2} e_{2} & <\beta_{3}-\beta_{2}<\left(q_{2}+1\right) e_{2}, \\
& \ldots \\
q_{u-1} e_{u-1} & <\beta_{u}-\beta_{u-1}<\left(q_{u-1}+1\right) e_{u-1}, \\
q_{u} e_{u} & <\beta_{u+1}-\beta_{u}<\left(q_{u}+1\right) e_{u},
\end{aligned}
$$

(2e) for $i=1,2, \ldots, u+1$, define $L_{i}(t)$ by

$$
\begin{aligned}
L_{1}(t) & =\sum_{i=1}^{q_{1}} b_{1 i} t^{i e_{1}} \in \mathbb{C}[t], \\
L_{2}(t) & =\sum_{i=1}^{q_{2}} b_{2 i} t^{i e_{2}} \in \mathbb{C}[t], \\
& \ldots \\
L_{u}(t) & =\sum_{i=1}^{q_{u}} b_{u i} t^{i e_{u}} \in \mathbb{C}[t], \\
L_{u+1}(t) & =\sum_{i=1}^{\infty} b_{u+1, i} t^{i} \in \mathbb{C}\{t\},
\end{aligned}
$$

such that all $b_{j, i(j)}$ are complex numbers with $1 \leq j \leq u+1$ and $1 \leq i(j) \leq q_{j}$, some of which may be zero. Note that $q_{u+1}$ may be infinite.

(3) Let the parametrization of $V(h)$ be defined by

$$
V(h):=\left\{\begin{aligned}
& y= c_{1} t^{l_{1}}\left(1+R_{1}(t)\right)+c_{2} t^{l_{2}}\left(1+R_{2}(t)\right) \\
&+\cdots+c_{v} t^{l_{v}}\left(1+R_{v}(t)\right)+c_{v+1} t^{l_{v+1}}\left(1+R_{v+1}(t)\right) \\
& z=t^{\gamma}
\end{aligned}\right.
$$

where

(3a) $2 \leq l_{1}<\gamma$ and $l_{1}<l_{2}<\cdots<l_{v+1}$,

(3b) $l_{1} \geq \operatorname{gcd}\left(\gamma, l_{1}\right)>\operatorname{gcd}\left(\gamma, l_{1}, l_{2}\right)>\cdots>\operatorname{gcd}\left(\gamma, l_{1}, l_{2}, \ldots, l_{v+1}\right)=1$,

(3c) the $c_{i}$ are all nonzero numbers for $i=1,2, \ldots, v+1$,

(3d) write $\tau_{1}=\operatorname{gcd}\left(\gamma, l_{1}\right), \tau_{2}=\operatorname{gcd}\left(\gamma, l_{1}, l_{2}\right), \ldots, \tau_{v}=\operatorname{gcd}\left(\gamma, l_{1}, l_{2}, \ldots, l_{v}\right)$, $\tau_{v+1}=\operatorname{gcd}\left(\gamma, l_{1}, l_{2}, \ldots, l_{v+1}\right)=1$, and define $\varepsilon_{1}, \varepsilon_{2}, \ldots, \varepsilon_{v}$ to be nonnegative in- 
tegers such that

$$
\begin{aligned}
\varepsilon_{1} \tau_{1} & <l_{2}-l_{1}<\left(\varepsilon_{1}+1\right) \tau_{1}, \\
\varepsilon_{2} \tau_{2} & <l_{3}-l_{2}<\left(\varepsilon_{2}+1\right) \tau_{2}, \\
& \ldots \\
\varepsilon_{v-1} \tau_{v-1} & <l_{v}-l_{v-1}<\left(\varepsilon_{v-1}+1\right) \tau_{v-1}, \\
\varepsilon_{v} \tau_{v} & <l_{v+1}-l_{v}<\left(\varepsilon_{v}+1\right) \tau_{v},
\end{aligned}
$$

(3e) for $i=1,2, \ldots, v+1$, define $R_{i}(t)$ by

$$
\begin{aligned}
R_{1}(t) & =\sum_{i=1}^{\varepsilon_{1}} c_{1 i} t^{i \tau_{1}} \in \mathbb{C}[t], \\
R_{2}(t) & =\sum_{i=1}^{\varepsilon_{2}} c_{2 i} t^{i \tau_{2}} \in \mathbb{C}[t], \\
& \cdots \\
R_{v}(t) & =\sum_{i=1}^{\varepsilon_{v}} c_{v i} t^{i \tau_{v}} \in \mathbb{C}[t], \\
R_{v+1}(t) & =\sum_{i=1}^{\infty} c_{v+1, i} t^{i} \in \mathbb{C}\{t\},
\end{aligned}
$$

such that all $c_{j, i(j)}$ are complex numbers with $1 \leq j \leq v+1$ and $1 \leq i(j) \leq \varepsilon_{j}$, some of which may be zero. Note that $\varepsilon_{v+1}$ may be infinite, and also that $\mathbb{C}[t]$ is the ring of polynomials in $t$ and $\mathbb{C}\{t\}$ is the ring of convergent power series at $t=0$.

\section{Conclusion We get the followings:}

(I) Note that $n>\operatorname{gcd}\left(n, \alpha_{1}\right)$ and $m>\operatorname{gcd}\left(m, \beta_{1}\right)$.

$$
V(f) \equiv V(g) \quad \text { (multi. seq.) }
$$

$\Longleftrightarrow$ the multiplicity and Puiseux exponents are the same, by Definition 4.1, that is, $n=m, r+1=u+1$, and $\alpha_{i}=\beta_{i} \quad$ for all $i=1,2, \ldots, r+1$,

$\Longleftrightarrow$ the Puiseux pairs for both $V(f)$ and $V(g)$ are the same.

(II) Let $\gamma>l_{1} \geq 2$. Then, it is enough to consider two cases: (IIa) $l_{1}>\operatorname{gcd}\left(\gamma, l_{1}\right)$ and (IIb) $l_{1}=\operatorname{gcd}\left(\gamma, l_{1}\right)$, that is, $l_{1}$ is a divisor of $\gamma$.

(IIa) Let $l_{1}>\operatorname{gcd}\left(\gamma, l_{1}\right)$.

$$
V(f) \equiv V(h) \quad \text { (multi. seq.) }
$$

$\Longleftrightarrow n=l_{1}, \alpha_{1}=\gamma, r+1=v+1$ and $\alpha_{i}=\gamma+l_{i}-l_{1} \quad$ for $1 \leq i \leq r+1$,

$\Longleftrightarrow$ the Puiseux pairs for both $V(f)$ and $V(h)$ are the same. 
(IIb) Let $l_{1}=\operatorname{gcd}\left(\gamma, l_{1}\right)$, that is, $l_{1}$ is a divisor of $\gamma$.

$$
V(f) \equiv V(h) \quad \text { (multi. seq.) }
$$

$\Longleftrightarrow n=l_{1}, \alpha_{1}=\gamma+l_{2}-l_{1}, r+1=v$ and $\alpha_{i}=\gamma+l_{i+1}-l_{1}$ for $2 \leq i \leq r+1$,

$\Longleftrightarrow$ the Puiseux pairs for both $V(f)$ and $V(h)$ are the same.

\section{COROLlary 5.2 .}

Assumption Suppose that the same assumption as in Theorem 5.1 are satisfied.

Conclusion Under the consequences of Theorem 5.1, we get the followings:

(I) Let $n>\operatorname{gcd}\left(n, \alpha_{1}\right)$ and $m>\operatorname{gcd}\left(m, \beta_{1}\right)$.

$$
\begin{aligned}
V(f) & \sim V(g) \\
\Longleftrightarrow V(f) & \equiv V(g) \quad \text { (multi. seq.). }
\end{aligned}
$$

Moreover, $V(f)$ is topologically equivalent to the curve defined by $y=t^{n}$ and $z=t^{\alpha_{1}}+t^{\alpha_{2}}+\cdots+t^{\alpha_{r+1}}$, which is the standard expansion for $V(f)$ by Definition 4.4.

(II) Let $\gamma>l_{1} \geq 2$. Then, there are two cases:

(IIa) $l_{1}>\operatorname{gcd}\left(\gamma, l_{1}\right)$ and (IIb) $l_{1}$ is a divisor of $\gamma$.

(IIa) Let $l_{1}>\operatorname{gcd}\left(\gamma, \dot{l}_{1}\right)$.

$$
\begin{aligned}
V(f) & \sim V(h) \\
\Longleftrightarrow V(f) & \equiv V(h) \quad \text { (multi. seq.). }
\end{aligned}
$$

Moreover, $V(h)$ can be topologically equivalent to the curve defined by any of the following two irreducible parametrizations (i) and (ii):

(i) $y=t^{l_{1}}+t^{l_{2}}+\cdots+t^{l_{v+1}}$ and $z=t^{\gamma}$.

(ii) $y=t^{l_{1}}$ and $z=t^{\gamma}+t^{\gamma+l_{2}-l_{1}}+t^{\gamma+l_{3}-l_{1}}+\cdots+t^{\gamma+l_{v+1}-l_{1}}$,

noting that the above (ii) is the standard Puiseux expansions for $V(h)$.

(IIb) Let $l_{1}=\operatorname{gcd}\left(\gamma, l_{1}\right)$, that is, $l_{1}$ is a divisor of $\gamma$.

$$
\begin{aligned}
V(f) & \sim V(h) \\
\Longleftrightarrow V(f) & \equiv V(h) \quad \text { (multi. seq.). }
\end{aligned}
$$

Moreover, $V(h)$ can be topologically equivalent to the curve defined by any of the following two irreducible parametrization (i) and (ii):

(i) $y=t^{l_{1}}+t^{l_{2}}+\cdots+t^{l_{v+1}}$ and $z=t^{\gamma}$.

(ii) $y=t^{l_{1}}$ and $z=\varepsilon t^{\gamma}+t^{\gamma+l_{2}-l_{1}}+t^{\gamma+l_{3}-l_{1}}+\cdots+t^{\gamma+l_{v+1}-l_{1}}$ for any number $\varepsilon$, noting that if $\varepsilon$ is a zero then the above (ii) is the standard Puiseux expansion for $V(h)$.

Proof of Theorem 5.1. It is enough to prove (I) and (II), respectively as follows:

(I) (5.1.4) is true, and (II) (5.1.5) and (5.1.6) are true, respectively. 
(I) In preparation for the proof of (5.1.4), first observe that the process of blow-ups and blow-downs preserve the multiplicity sequences of irreducible plane curve singularities. First of all, we are going to prove by induction on the multiplicity of the curve $V(f)$ that if $V(f) \equiv V(g) \quad$ (multi. seq.) where $V(f)$ and $V(g)$ are defined by (5.1.1) and (5.1.2), respectively, then the Puiseux pairs for both $V(f)$ and $V(g)$ are the same. After then, the converse will be proved.

As we have seen in (5.1.1) of this assumption, for example, recall that the parametrization of the irreducible curve $V(f)$ defined by a given analytic function $f$ in $\mathbb{C}\{y, z\}$ was rewritten in the same form as follows:

$$
V(f):=\left\{\begin{array}{l}
y=t^{n} \\
z=\sum_{i=1}^{r+1} a_{i} t^{\alpha_{i}}\left(1+D_{i}(t)\right)
\end{array}\right.
$$

with the same properties (1a), (1b), (1c), (1d) and (1e) of (5.1.1). Since $2 \leq n<\alpha_{1}$ and $n>\operatorname{gcd}\left(n, \alpha_{1}\right)$ by (1a) and (1b), then there is a positive integer $\sigma$ such that $n \sigma<\alpha_{1}<n(\sigma+1)$.

Now, we can take $\sigma$ iterations of blow-ups in process of the resolution of the singular point $(0,0)$ of $V(f)$ in an elementary way, and then construct inductively the local defining equation for $V^{(k)}(f)$, the $k$-th proper transform of $V(f)$ under $k$-times of blow-ups with $1 \leq k \leq \sigma$ as follows:

(i) Let $\alpha_{1}-n>0$ or $\sigma \geq 1$. Let $\pi_{1}: M^{(1)} \rightarrow \mathbb{C}^{2}$ be a blow-up of $\mathbb{C}^{2}$ at $(y, z)=(0,0)$ which is a singular point of $V(f)$. Let $\left(v_{1}, u_{1}\right)$ and $\left(v_{1}^{\prime}, u_{1}^{\prime}\right)$ be the local coordinates for $M^{(1)}$ with $\pi_{1}\left(v_{1}, u_{1}\right)=(y, z)=\left(v_{1}, v_{1} u_{1}\right)$ and $\pi_{1}\left(v_{1}^{\prime}, u_{1}^{\prime}\right)=(y, z)=$ $\left(v_{1}^{\prime} u_{1}^{\prime}, v_{1}^{\prime}\right)$ where $u_{1}^{\prime}=\frac{1}{u_{1}}$ and $v_{1}^{\prime}=v_{1} u_{1}$. Since $f$ is irreducible in $\mathbb{C}\{y, z\}$ and $n<\alpha_{1}$, then just one coordinate patch of the local coordinates, i.e., $\left(v_{1}, u_{1}\right)$ is needed for the study of the proper transform $V^{(1)}(f)$ of $V(f)$ at $(y, z)=(0,0)$ under $\pi_{1}$. Then, the local defining equation for $V^{(1)}(f)$ at $\left(v_{1}, u_{1}\right)=(0,0)$ is written in the form

$$
V^{(1)}(f):=\left\{\begin{array}{l}
v_{1}=t^{n} \\
u_{1}=\sum_{i=1}^{r+1} a_{i} t^{\alpha_{i}-n}\left(1+D_{i}(t)\right)
\end{array} .\right.
$$

If $0<\alpha_{1}-n<n$ or $\sigma=1$, then we do not take the next step.

If $\alpha_{1}-n>n$ or $\sigma \geq 2$, then take the next step.

(ii) Let $\alpha_{1}-2 n>0$ or $\sigma \geq 2$. Then, $V^{(1)}(f)$ has a singular point at $\left(v_{1}, u_{1}\right)=$ $(0,0)$. Let $\pi_{2}: M^{(2)} \rightarrow M^{(1)}$ be a blow-up of $M^{(1)}$ at $\left(v_{1}, u_{1}\right)=(0,0)$, and let $\left(v_{2}, u_{2}\right)$ and $\left(v_{2}^{\prime}, u_{2}^{\prime}\right)$ be the local coordinates for $M^{(2)}$ with $\pi_{2}\left(v_{2}, u_{2}\right)=\left(v_{1}, u_{1}\right)=\left(v_{2}, v_{2} u_{2}\right)$ and $\pi_{2}\left(v_{2}^{\prime}, u_{2}^{\prime}\right)=\left(v_{1}, u_{1}\right)=\left(v_{2}^{\prime} u_{2}^{\prime}, v_{2}^{\prime}\right)$ where $u_{2}^{\prime}=\frac{1}{u_{2}}$ and $v_{2}^{\prime}=v_{2} u_{2}$. Since $f$ is irreducible in $\mathbb{C}\{y, z\}$ and $2 n<\alpha_{1}$, then just one of the local coordinates, i.e., $\left(v_{2}, u_{2}\right)$ is needed for the study of $V^{(2)}(f)$, i.e., the proper transform of $V(f)$ at $\left(v_{2}, u_{2}\right)=(0,0)$ under $\pi_{1} \circ \pi_{2}$. Then, the local defining equation for $V^{(2)}(f)$ is written in the form

$$
V^{(2)}(f):=\left\{\begin{array}{l}
v_{2}=t^{n} \\
u_{2}=\sum_{i=1}^{r+1} a_{i} t^{\alpha_{i}-2 n}\left(1+D_{i}(t)\right)
\end{array} .\right.
$$


If $0<\alpha_{1}-2 n<n$ or $\sigma=2$, then we do not take the next step.

If $\alpha_{1}-2 n>n$ or $\sigma \geq 3$, then take the next step.

......

(k) Let $\alpha_{1}-k n>0$ or $\sigma \geq k$. Then, by induction on the positive integer $\sigma$, $V^{(k-1)}(f)$ has a singular point at $\left(v_{k-1}, u_{k-1}\right)=(0,0)$. Let $\pi_{k}: M^{(k)} \rightarrow M^{(k-1)}$ be a blow-up of $M^{(k-1)}$ at $\left(v_{k-1}, u_{k-1}\right)=(0,0)$, and let $\left(v_{k}, u_{k}\right)$ and $\left(v_{k}^{\prime}, u_{k}^{\prime}\right)$ be the local coordinates for $M^{(k)}$ with $\pi_{k}\left(v_{k}, u_{k}\right)=\left(v_{k-1}, u_{k-1}\right)=\left(v_{k}, v_{k} u_{k}\right)$ and $\pi_{k}\left(v_{k}^{\prime}, u_{k}^{\prime}\right)=$ $\left(v_{k-1}, u_{k-1}\right)=\left(v_{k}^{\prime} u_{k}^{\prime}, v_{k}^{\prime}\right)$ where $u_{k}^{\prime}=\frac{1}{u_{k}}$ and $v_{k}^{\prime}=v_{k} u_{k}$. Since $f$ is irreducible in $\mathbb{C}\{y, z\}$ and $k n<\alpha_{1}$, then just one of the local coordinates, i.e., $\left(v_{k}, u_{k}\right)$ is needed for the study of $V^{(k)}(f)$, that is, the proper transform of $V(f)$ at $(0,0)$ under $\pi_{1} \circ \pi_{2} \circ$ $\cdots \circ \pi_{k}$.

If $0<\alpha_{1}-k n<n$ or $\sigma=k$, then we do not take the next step.

If $\alpha_{1}-k n>n$ or $\sigma \geq k+1$, then take the next step.

Thus, it can be easily shown that the local defining equation for $V^{(k)}(f)$ is written as follows:

$$
V^{(k)}(f):=\left\{\begin{array}{l}
v_{k}=t^{n} \\
u_{k}=\sum_{i=1}^{r+1} a_{i} t^{\alpha_{i}-k n}\left(1+D_{i}(t)\right)
\end{array}\right.
$$

for $k=1,2, \ldots, \sigma$ and that $\alpha_{1}-n>n, \ldots, \alpha_{1}-n(\sigma-1)>n$, but $n>\alpha_{1}-n \sigma>0$. Note that the multiplicity sequence is nonincreasing for irreducible plane curve singularites. Therefore, summarizing the above results, we get easily the followings:

Sublemma 5.1.1. Suppose that $V(f)$ satisfies the same assumptions and notations in this theorem. By assumption, there is a positive integer $\sigma$ such that $n \sigma<$ $\alpha_{1}<n(\sigma+1)$.

As a conclusion, $V^{(\sigma)}(f)$ is the first appearing proper transform among all proper transforms, which are defined in the standard resolution process of the singular point of $V(f)$ such that the multiplicity of each proper transform is less than mult $(V(f),(0,0))=n$. Also, $\left(v_{\sigma}, u_{\sigma}\right)=(0,0)$ is either the singular point of $V^{(\sigma)}(f)$ or the nonsingular point at which is needed only to get additional blow-ups for the standard resolution of the singular point of $V(f)$, as we have seen in (5.1.10).

Now for proof of (5.1.4) in (I), to show that we may begin with the assumption that $n=m$ and $\alpha_{1}=\beta_{1}$, then it is enough to prove the following claim:

$$
\text { if } f \equiv g \text { (mult. seq.), then } n=m \text { and } \alpha_{1}=\beta_{1} \text {. }
$$

If $f \equiv g$ (multi. seq.), then it is trivial that $n=m$. If $f \equiv g$ (multi. seq.), then to prove that $\alpha_{1}=\beta_{1}$, let $\delta$ be the positive integer such that $m \delta<\beta_{1}<m(\delta+1)$ and let $m=n$ because $g$ satisfies the same kind of assumptions and notations as $f$ does in the theorem. After $\delta$ iterations of blow-ups at the singular point $(0,0)$ of $V(g)$ as we have done in the resolution process of singular point of $V(f)$, the local defining equation for the $k-t h$ proper transform $V^{(k)}(g)$ of $V(g)$ with $1 \leq k \leq \delta$ can be written as 
follows:

$$
V^{(k)}(g):=\left\{\begin{array}{l}
\bar{v}_{k}=t^{n} \\
\bar{u}_{k}=\sum_{i=1}^{u+1} b_{i} t^{\beta_{1}-k n}\left(1+L_{i}(t)\right)
\end{array}\right.
$$

where

(i) $\left(\bar{v}_{\delta}, \bar{u}_{\delta}\right)$ is defined to be one of the local coordinates for the $\delta-t h$ blow-up $\bar{\pi}_{\delta}: \bar{M}^{(\delta)} \rightarrow \bar{M}^{(\delta-1)}$, which is defined similarly as we have seen in the process of blow-ups for $V(f)$,

(ii) $V^{(\delta)}(g)$ is the first appearing proper transform, among all proper transforms which are defined in the standard resolution process of the singular point of $V(g)$ such that the multiplicity of each proper transform is less than $n=\operatorname{mult}(V(g),(0,0))$. If $\delta \leq \sigma$, then $\bar{\pi}_{k}$ and $\left(\bar{v}_{k}, \bar{u}_{k}\right)$ can be identified with $\pi_{k}$ and $\left(v_{k}, u_{k}\right)$ for $1 \leq k \leq \delta$, respectively.

Thus, if $f \equiv g$ (mult. seq.), then by Sublemma 5.1.1 $n=m, \sigma=\delta$ and so $\alpha_{1}=\beta_{1}$. Therefore, to prove (5.1.4) in (I), we may begin with assumption that $n=m$ and $\alpha_{1}=\beta_{1}$.

Now, the proof will be by induction on the multiplicity $n$ of the local defining equation $f(y, z)$ at the origin for $V(f)$. Then, it is enough to consider the following two cases, respectively: (I-1) $n=2$ and $(\mathrm{I}-2) n \geq 2$.

(I-1) If $n=2$, then the local defining equation for $V(f)$ can be described by

$$
V(f):=\left\{\begin{array}{l}
y=t^{2} \\
z=a_{1} t^{\alpha_{1}}+\sum_{i>\alpha_{1}} A_{i} t^{i}
\end{array}\right.
$$

where $\operatorname{gcd}\left(2, \alpha_{1}\right)=1, a_{1}$ is a nonzero constant and the $A_{i}$ are complex numbers. If the $A_{i}$ are all zero, then it is clear that

$$
V(f) \equiv\left\{(y, z): y=t^{2} \text { and } z=t^{\alpha_{1}}\right\} \quad \text { (mult. seq.). }
$$

If some $A_{i}$ are nonzero, then it is easily shown that $z=a_{1} t^{\alpha_{1}}+\sum_{i>\alpha_{1}} A_{i} t^{i}$ is rewritten in the form

$$
z=a_{1} t^{\alpha_{1}}\left(1+\sum_{2 i>0} B_{i} t^{2 i}\right)+\sum_{2 j>\alpha_{1}} C_{j} t^{2 j}
$$

where the $B_{i}$ and the $C_{j}$ are complex numbers. Then, observe that (5.1.15) can be rewritten in the form

$$
z=a_{1} t^{\alpha_{1}}\left(1+\sum_{2 i>0} B_{i} y^{i}\right)+\sum_{2 j>\alpha_{1}} C_{j} y^{j}
$$

So, define $z^{\prime}=\frac{\left(z-\sum_{2 j>\alpha_{1}} C_{j} y^{j}\right)}{\left(1+\sum_{2 i>0} B_{i} y^{i}\right)}$, and then $V(f)$ and $\left\{\left(y, z^{\prime}\right): y=t^{2}, z^{\prime}=a_{1} t^{\alpha_{1}}\right\}$ have the same multiplicity sequence because these two varieties are analytically equivalent at the origin. In this case, there is nothing to prove by (5.1.13).

(I-2) Suppose by the induction assumption that this conclusion is true if the multiplicity of $f$ at the origin is either less than $n$ or equal to two. For the proof, we 
may assume that the multiplicity of both $f$ and $g$ at $(y, z)=(0,0)$ is $n$, and also that $\alpha_{1}=\beta_{1}$ as we have done in the beginning of the proof because $n<\alpha_{1}, n<\beta_{1}, n \backslash \alpha_{1}$ and $n \backslash \beta_{1}$. Just as before, let $\sigma$ be the positive integer such that $n \sigma<\alpha_{1}<n(\sigma+1)$. Then, the local defining equations for the $\sigma-t h$ proper transforms $V^{(\sigma)}(f)$ and $V^{(\sigma)}(g)$ can be written, respectively as follows: Note that $\alpha_{1}=\beta_{1}$.

$$
\begin{aligned}
& V^{(\sigma)}(f):=\left\{\begin{array}{l}
v_{\sigma}=t^{n} \\
u_{\sigma}=\sum_{i=1}^{r+1} a_{i} t^{\alpha_{i}-\sigma n}\left(1+D_{i}(t)\right)
\end{array}\right. \\
& V^{(\sigma)}(g):=\left\{\begin{array}{l}
v_{\sigma}=t^{n} \\
u_{\sigma}=\sum_{i=1}^{u+1} b_{i} t^{\beta_{i}-\sigma n}\left(1+L_{i}(t)\right)
\end{array}\right.
\end{aligned}
$$

where $\pi_{\sigma}: M^{(\sigma)} \rightarrow M^{(\sigma-1)}$ is a blow-up of $M^{(\sigma-1)}$ at $\left(v_{\sigma-1}, u_{\sigma-1}\right)=(0,0)$, which was already defined in the proof of Sublemma 5.1.1.

Note that irreducible parametrizations for local defining equations in (5.1.17) and (5.1.18) are not Puiseux expansion because $\alpha_{1}-n \sigma<n$.

So, for the induction proof, we are going to construct two Puiseux expansion, which are equivalent for local defining equations in (5.1.17) and (5.1.18), respectively. In order to use the conclusion in Theorem 3.4, we are going to prove that two local defining equations in (5.1.17) and (5.1.18) satisfy the assumptions of Theorem 3.4 respectively. For this purpose, it is just enough to observe the followings with $\operatorname{Remark}(*)$ : Note that $\alpha_{1}=\beta_{1}$.

(1)(1a) $2 \leq n$ and $1 \leq \alpha_{1}-n \sigma<\alpha_{2}-n \sigma<\cdots<\alpha_{r+1}-n \sigma$,

(1b) $n>\operatorname{gcd}\left(n, \alpha_{1}-n \sigma\right)>\cdots>\operatorname{gcd}\left(n, \alpha_{1}-n \sigma, \ldots, \alpha_{r+1}-n \sigma\right)=1$,

(1c) the $a_{i}$ are all nonzero numbers for $i=1,2, \ldots, r+1$.

(2)(2a) $2 \leq n$ and $1 \leq \beta_{1}-n \sigma<\beta_{2}-n \sigma<\cdots<\beta_{r+1}-n \sigma$,

(2b) $n>\operatorname{gcd}\left(n, \beta_{1}-n \sigma\right)>\cdots>\operatorname{gcd}\left(n, \beta_{1}-n \sigma, \ldots, \beta_{r+1}-n \sigma\right)=1$,

(2c) the $b_{i}$ are all nonzero numbers for $i=1,2, \ldots, r+1$.

$\operatorname{Remark}(*)$ : If $\alpha_{1}-\sigma n=1$ in (1a), then $\operatorname{gcd}\left(n, \alpha_{1}\right)=1$, and so there are no more exponents $\alpha_{i}$ of the parameter $t$ for $i \geq 2$ in the parametrization for the curve $V(f)$. In this case, there is nothing to prove, because $\alpha_{1}=\beta_{1}$. That is, the same multiplicity sequence implies the invariance of the multiplicity and Puiseux exponents.

Therefore, for the proof, we may assume without loss of generality that $\alpha_{1}-\sigma n=$ $\beta_{1}-\sigma n \geq 2$ in (1a) and (2a).

Now, by the same method as we have used in the either conclusion or proof of Theorem 3.4, we can construct the new parameters for $V^{(\sigma)}(f)$ and $V^{(\sigma)}(g)$, respectively as follows:

For convenience of the representation, the notations of these new parameters and so on, can be suitably chosen the same for both $V^{(\sigma)}(f)$ and $V^{(\sigma)}(g)$, if there is no complexity.

(5.1.19) Define a new parameter $s=\phi(t)$ by a conformal mapping of $t$ at the origin such that $s(0)=0$ and $u_{\sigma}=s^{\alpha_{1}-\sigma n}$ where $\left(v_{\sigma}, u_{\sigma}\right)$ is one of a given local coordinates in (5.1.17). 
(5.1.20) Define a new parameter $s=\psi(t)$ by a conformal mapping of $t$ at the origin such that $s(0)=0$ and $u_{\sigma}=s^{\beta_{1}-\sigma n}$ where $\left(v_{\sigma}, u_{\sigma}\right)$ is one of a given local coordinates in (5.1.18).

In preparation for applying the induction assumption to the proof, first it can be easily shown by Theorem 3.4 and by a new parameter $s$ in (5.1.19) and (5.1.20) that the local defining equations for $V^{(\sigma)}(f)$ of $(5.1 .17)$ and $V^{(\sigma)}(g)$ of $(5.1 .18)$ are analytically written, respectively as follows: Note that $\alpha_{1}=\beta_{1}$.

$$
\begin{aligned}
& V^{(\sigma)}(f): \approx\left\{\begin{array}{l}
v_{\sigma}=\sum_{i=1}^{r+1} \zeta_{i} s^{n+\alpha_{i}-\alpha_{1}}\left(1+\bar{D}_{i}(s)\right) \\
u_{\sigma}=s^{\alpha_{1}-\sigma n}
\end{array}\right. \\
& V^{(\sigma)}(g): \approx\left\{\begin{array}{l}
v_{\sigma}=\sum_{i=1}^{u+1} \eta_{i} s^{n+\beta_{i}-\beta_{1}}\left(1+\bar{L}_{i}(s)\right) \\
u_{\sigma}=s^{\beta_{1}-\sigma n}
\end{array}\right.
\end{aligned}
$$

where

(i) for $i=1,2, \ldots, r+1$, the $\zeta_{i}$ are all nonzero numbers, and define $\bar{D}_{i}(s)$ by

$$
\begin{gathered}
\bar{D}_{1}(s)=\sum_{i=1}^{p_{1}} \bar{a}_{1 i} s^{i d_{1}} \in \mathbb{C}[s] \\
\bar{D}_{2}(s)=\sum_{i=1}^{p_{2}} \bar{a}_{2 i} s^{i d_{2}} \in \mathbb{C}[s] \\
\ldots \ldots \\
\bar{D}_{r}(s)=\sum_{i=1}^{p_{r}} \bar{a}_{r i} s^{i d_{r}} \in \mathbb{C}[s] \\
\bar{D}_{r+1}(s)=\sum_{i=1}^{\infty} \bar{a}_{r+1, i} s^{i} \in \mathbb{C}\{s\}
\end{gathered}
$$

such that all $\bar{a}_{j, i(j)}$ are complex numbers with $1 \leq j \leq r+1$ and $1 \leq i(j) \leq p_{j}$, some of which may be zero.

(ii) for $i=1,2, \ldots, u+1$, the $\eta_{i}$ are all nonzero numbers, and define $\bar{L}_{i}(s)$ by

$$
\begin{aligned}
\bar{L}_{1}(s) & =\sum_{i=1}^{q_{1}} \bar{b}_{1 i} s^{i e_{1}} \in \mathbb{C}[s] \\
\bar{L}_{2}(s) & =\sum_{i=1}^{q_{2}} \bar{b}_{2 i} s^{i e_{2}} \in \mathbb{C}[s] \\
& \cdots \\
\bar{L}_{u}(s) & =\sum_{i=1}^{q_{u}} \bar{b}_{u, i} s^{i e_{u}} \in \mathbb{C}[s] \\
\bar{L}_{u+1}(s) & =\sum_{i=1}^{\infty} \bar{b}_{u+1, i} s^{i} \in \mathbb{C}\{s\},
\end{aligned}
$$


such that all $\bar{b}_{j, i(j)}$ are all complex numbers with $1 \leq j \leq u+1$ and $1 \leq i(j) \leq q_{j}$, some of which may be zero.

Then, it is enough to consider the following two cases: Note that $\alpha_{1}-\sigma n \geq 2$.

(A) $\left(\alpha_{1}-\sigma n\right) \not / n$ and (B) $\left(\alpha_{1}-\sigma n\right) \mid n$.

Case (A): Assume that $\alpha_{1}-\sigma n$ is not a divisor of $n$. Using (5.1.21) and (5.1.23), to prove by induction assumption on the multiplicity on the curve $V(f)$ that $V^{(\sigma)}(f)$ satisfies the same kind of properties in terms of coordinates $\left(v_{s}, u_{s}\right)$ as $V(f)$ does in the coordinates $(y, z)$ in the assumption of Theorem 5.1, first of all, we need to claim the following properties:

(i) $2 \leq \alpha_{1}-\sigma n<n+\alpha_{1}-\alpha_{1}<n+\alpha_{2}-\alpha_{1}<\cdots<n+\alpha_{r+1}-\alpha_{1}$.

(ii) Write $\omega_{1}=\operatorname{gcd}\left(\alpha_{1}-\sigma n, n+\alpha_{1}-\alpha_{1}\right), \omega_{2}=\operatorname{gcd}\left(\alpha_{1}-\sigma n, n+\alpha_{1}-\alpha_{1}, n+\right.$ $\left.\alpha_{2}-\alpha_{1}\right), \ldots, \omega_{r}=\operatorname{gcd}\left(\alpha_{1}-\sigma n, n+\alpha_{1}-\alpha_{1}, n+\alpha_{2}-\alpha_{1}, \ldots, n+\alpha_{r}-\alpha_{1}\right), \omega_{r+1}=$ $\operatorname{gcd}\left(\alpha_{1}-\sigma n, n+\alpha_{1}-\alpha_{1}, n+\alpha_{2}-\alpha_{1}, \ldots, n+\alpha_{r+1}-\alpha_{1}\right)$, and then $\alpha_{1}-\sigma n>\omega_{1}>$ $\omega_{2}>\omega_{3}>\cdots>\omega_{r+1}=1$.

(iii) The $\zeta_{i}$ are all nonzero numbers for $1=1,2, \ldots, r+1$.

(iv) Following the notations in (ii), define $\bar{p}_{1}, \bar{p}_{2}, \ldots, \bar{p}_{r}$ to be nonnegative integers such that for $i=1,2, \ldots, r$,

$$
\bar{p}_{i} \omega_{i}<\left(n+\alpha_{i+1}-\alpha_{1}\right)-\left(n+\alpha_{i}-\alpha_{1}\right)<\left(\bar{p}_{i}+1\right) \omega_{i} .
$$

(v) For $j=1,2, \ldots, r+1, \bar{D}_{j}(s)$ of (5.1.23) may be written in the form

$$
\bar{D}_{j}(s)=\sum_{i=1}^{\bar{p}_{j}} \bar{a}_{j i} s^{i \omega_{j}} .
$$

For proof of the claim, we prove each of the above properties (i), (ii), (iii), (iv) and (v) in the following way:

To prove (i), note that $\sigma$ is the positive integer such that $n \sigma<\alpha_{1}<n(\sigma+1)$ and that $\alpha_{i+1}>\alpha_{i}$ for $1 \leq i \leq r$, and so it is trivial.

To prove (ii), it is clear that $\omega_{1}=\operatorname{gcd}\left(\alpha_{1}-n \sigma, n\right)=\operatorname{gcd}\left(\alpha_{1}, n\right), \omega_{2}=\operatorname{gcd}\left(\alpha_{1}-\right.$ $\left.n \sigma, n, n+\alpha_{2}-\alpha_{1}\right)=\operatorname{gcd}\left(\alpha_{1}, n, \alpha_{2}\right), \ldots, \omega_{r+1}=\operatorname{gcd}\left(\alpha_{1}-n \sigma, n, n+\alpha_{2}-\alpha_{1}, \ldots, n+\right.$ $\left.\alpha_{r+1}-\alpha_{1}\right)=\operatorname{gcd}\left(\alpha_{1}, n, \alpha_{2}, \ldots, \alpha_{r+1}\right)$. Also, by following the definition of $d_{i}$ in both (1b) and (1d) of (5.1.1), $\omega_{i}=d_{i}$, and so $d_{i}>d_{i+1}$ implies that $\omega_{i}>\omega_{i+1}$ for $1 \leq i \leq r$.

So, it remains to prove that $\alpha_{1}-n \sigma>\omega_{1}=d_{1}$. Note by definition of $\sigma$ that $\alpha_{1}-n \sigma<n$. Now, assume the contrary. Then, $\alpha_{1}-n \sigma=\operatorname{gcd}\left(\alpha_{1}-n \sigma, n\right)=d_{1}<n$, and so $\alpha_{1}-n \sigma$ would be a divisor of $n$, which gives a contradiction to the assumption. Thus, the proof is done.

To prove (iii) is clear.

Finally, to prove (iv) and (v), it was already proved by (ii) that $d_{i}=\omega_{i}$, and then $\bar{p}_{i}$ can be replaced by $p_{i}$ for $i=1,2, \ldots, r+1$, just considering that $\left(n+\alpha_{j+1}-\alpha_{1}\right)-$ $\left(n+\alpha_{j}-\alpha_{1}\right)=\alpha_{j+1}-\alpha_{j}$ from (5.1.25). Therefore, the proof of (i), (ii), (iii), (iv) and $(\mathrm{v})$ are finished.

Also, since it can be assumed that $n=m$ and $\alpha_{1}=\beta_{1}$, then note that $\beta_{1}-\sigma m$ is not a divisor of $m$. So, by the similar method as above, it can be easily shown that $V^{(\sigma)}(g)$ satisfies the same kind of properties in terms of coordinates $\left(u_{\sigma}, v_{\sigma}\right)$ as $V(g)$ does in the local coordinates $(y, z)$. Since it was already shown that $V^{(k)}(f)$ and 
$V^{(k)}(g)$ have the same multiplicity $n$ at $\left(v_{k}, u_{k}\right)=(0,0)$ for $k=1,2, \ldots, \sigma$ as we have done in (5.1.10), then we have

$$
\begin{aligned}
& V(f) \equiv V(g) \quad \text { (multi. seq.) } \\
\Longrightarrow \quad & V^{(\sigma)}(f) \equiv V^{(\sigma)}(g) \quad \text { (multi. seq.). }
\end{aligned}
$$

Since the multiplicity of $V^{(\sigma)}(f)$ at $\left(v_{\sigma}, u_{\sigma}\right)=(0,0)$ is $\alpha_{1}-\sigma n$, which is less than $n$ and also the multiplicity of $V^{(\sigma)}(g)$ at $\left(v_{\sigma}, u_{\sigma}\right)=(0,0)$ is $\beta_{1}-\sigma n=\alpha_{1}-\sigma n$, then the induction assumption in both (5.1.21) and (5.1.22) implies that

$$
\begin{aligned}
& V^{(\sigma)}(f) \equiv V^{(\sigma)}(g) \quad \text { (multi. seq.) } \\
\Longrightarrow & \alpha_{1}-\sigma n=\beta_{1}-\sigma n, n=m, r+1=u+1 \text { and } \\
& n+\alpha_{i}-\alpha_{1}=n+\beta_{i}-\beta_{1} \quad \text { for } 1 \leq i \leq r+1 \\
\Longrightarrow & n=m, r+1=u+1, \quad \text { and } \alpha_{i}=\beta_{i} \quad \text { for } 1 \leq i \leq r+1 .
\end{aligned}
$$

In this case, we proved by (5.1.27) that

$$
\begin{aligned}
& V(f) \equiv V(g) \quad \text { (multi. seq.) } \\
\Longrightarrow & n=m, r+1=u+1, \quad \text { and } \alpha_{i}=\beta_{i} \quad \text { for } 1 \leq i \leq r+1 .
\end{aligned}
$$

Thus, the proof in Case (A) is completely done.

Case (B): Let $\alpha_{1}-\sigma n$ be a divisor of $n$. We may start with the assumption that $n=\overline{m \text { and } \alpha_{1}}=\beta_{1}$ with $\alpha_{1}-\sigma n \geq 2$.

So, it is enough to consider the following case:

$$
d_{1}=\operatorname{gcd}\left(n, \alpha_{1}\right)=\alpha_{1}-\sigma n \geq 2,
$$

because $\operatorname{gcd}\left(n, \alpha_{1}\right)=\operatorname{gcd}\left(n, \alpha_{1}-n \sigma\right)=\alpha_{1}-n \sigma$.

Recall by assumption that there is a positive integer $\sigma$ such that $n \sigma<\alpha_{1}<n(\sigma+$ 1). Since it was already shown that $V^{(k)}(f)$ and $V^{(k)}(g)$ have the same multiplicity $n$ at $\left(v_{k}, u_{k}\right)=(0,0)$ for $k=1,2, \ldots, \sigma$ as we have done in (5.1.10), then we have

$$
\begin{aligned}
& V(f) \equiv V(g) \quad \text { (multi. seq.) } \\
\Longrightarrow & V^{(\sigma)}(f) \equiv V^{(\sigma)}(g) \quad \text { (multi. seq.). }
\end{aligned}
$$

From the local defining equations of $V^{(\sigma)}(f)$ of $(5.1 .21)$ and $V^{(\sigma)}(g)$ of (5.1.22), for the remaining proof, it suffices to show that the following change of coordinates is nonsingular: Note that $\alpha_{1}-\sigma n$ is a divisor of $n$.

$$
\begin{aligned}
V^{(\sigma)}(f) & \Longrightarrow\left\{\begin{array}{l}
v_{\sigma}^{\prime}=\sum_{i=2}^{r+1} \zeta_{i} s^{n+\alpha_{i}-\alpha_{1}}\left(1+\bar{D}_{i}(s)\right) \\
u_{\sigma}^{\prime}=s^{\alpha_{1}-\sigma n}
\end{array}\right. \\
V^{(\sigma)}(g) & \Longrightarrow\left\{\begin{array}{l}
v_{\sigma}^{\prime \prime}=\sum_{i=2}^{\mu+1} \eta_{i} s^{n+\beta_{i}-\beta_{1}}\left(1+\bar{L}_{i}(s)\right) \\
u_{\sigma}^{\prime \prime}=s^{\beta_{1}-\sigma n}
\end{array}\right.
\end{aligned}
$$

where $v_{\sigma}^{\prime}=v_{\sigma}-\zeta_{1} s^{n}\left(1+\bar{D}_{1}(s)\right)$ with $u_{\sigma}^{\prime}=u_{\sigma}$, and $v_{\sigma}^{\prime \prime}=v_{\sigma}-\eta_{1} s^{n}\left(1+\bar{L}_{1}(s)\right)$ with $u_{\sigma}^{\prime \prime}=u_{\sigma}$. 
Since $d_{1}=\alpha_{1}-\sigma n$ is a divisor of $n$ by (5.1.30), then write $n=\varepsilon d_{1}$ for some positive integer $\varepsilon$. Then, $u_{\sigma}=s^{\alpha_{1}-\sigma n}=s^{d_{1}}$ by (5.1.21), and also $u_{\sigma}^{n}=s^{\varepsilon d_{1}}$. So, $\bar{D}_{1}(s)=\sum_{i=1}^{p_{1}} \bar{a}_{1 i} s^{i d_{1}} \in \mathbb{C}[s]$ of (5.1.23) implies that $v_{\sigma}^{\prime}=v_{\sigma}-\zeta_{1} s^{n}\left(1+\bar{D}_{1}(s)\right)$ is written in the form

$$
v_{\sigma}^{\prime}=v_{\sigma}-\zeta_{1} u_{\sigma}^{\varepsilon}\left(1+F\left(u_{\sigma}\right)\right)
$$

where $F\left(u_{\sigma}\right)$ is a polynomial in $\mathbb{C}\left[u_{\sigma}\right]$ with $F\left(u_{\sigma}\right)=\sum_{i=1}^{p_{1}} \bar{a}_{1 i} u_{\sigma}{ }^{i} \in \mathbb{C}\left[u_{\sigma}\right]$.

Since it is trivial to show by Lemma 4.3 that an analytic mapping $\psi$ from $\left(v_{\sigma}, u_{\sigma}\right)$ to $\left(v_{\sigma}^{\prime}, u_{\sigma}^{\prime}\right)$ is a nonsingular change of coordinates at $\left(v_{\sigma}, u_{\sigma}\right)=(0,0)$ where $v_{\sigma}^{\prime}=$ $v_{\sigma}-\zeta_{1} u_{\sigma}^{\varepsilon}\left(1+F\left(u_{\sigma}\right)\right)$ and $u_{\sigma}^{\prime}=u_{\sigma}$ with $\psi(0,0)=(0,0)$, then $V^{(\sigma)}(f)$ is analytically well-defined by

$$
V^{(\sigma)}(f): \approx\left\{\begin{array}{l}
v_{\sigma}^{\prime}=\sum_{i=2}^{r+1} \zeta_{i} s^{n+\alpha_{i}-\alpha_{1}}\left(1+\bar{D}_{i}(s)\right) \\
u_{\sigma}^{\prime}=s^{\alpha_{1}-\sigma n} .
\end{array}\right.
$$

That is, $V^{(\sigma)}(f)$ of (5.1.34) satisfies the same kind of assumptions relative to the local coordinates $\left(v_{\sigma}^{\prime}, u_{\sigma}^{\prime}\right)$ as $V^{(\sigma)}(f)$ of $(5.1 .21)$ has done relative to the local coordinates $\left(v_{\sigma}, u_{\sigma}\right)$.

Also, by the same method just as we have done in (5.1.34), using a nonsingular change of coordinates for $V^{(\sigma)}(g)$ at $\left(v_{\sigma}^{\prime \prime}, u_{\sigma}^{\prime \prime}\right)$, then $V^{(\sigma)}(g)$ can be analytically written in the form

$$
V^{(\sigma)}(g): \approx\left\{\begin{array}{l}
v_{\sigma}^{\prime \prime}=\sum_{i=2}^{u+1} \eta_{i} s^{n+\beta_{i}-\beta_{1}}\left(1+\bar{L}_{1}(s)\right) \\
u_{\sigma}^{\prime \prime}=s^{\beta_{1}-\sigma n} .
\end{array}\right.
$$

Since $d_{1}=\alpha_{1}-\sigma n>d_{2}=\operatorname{gcd}\left(\alpha_{1}-\sigma n, n+\alpha_{2}-\alpha_{1}\right)$ and $d_{1}=\left(\beta_{1}-\sigma n\right)>$ $\operatorname{gcd}\left(\beta_{1}-\sigma n, n+\beta_{2}-\beta_{1}\right)$, then by the same method as we have done in Case (A), it is trivial to show that

$$
\begin{aligned}
V^{(\sigma)}(f) & \equiv V^{(\sigma)}(g) \quad \text { (multi. seq.) } \\
\Longrightarrow \alpha_{1}-\sigma n & =\beta_{1}-\sigma n, \quad r=u \text { and } n+\alpha_{i}-\alpha_{1}=n+\beta_{i}-\beta_{1} \text { for } 2 \leq i \leq r+1 .
\end{aligned}
$$

Thus, we proved by (5.1.36) that whenever $V(f)$ and $V(g)$ have the same multiplicity sequence for Case (B), then $n=m, r+1=u+1$ and $\alpha_{i}=\beta_{i}$ for $i=1,2, \ldots, r+1$.

Therefore, summarizing the results of Case (A) and Case (B), then the proof of the sufficiency for (I) is finished.

Next, to prove the converse for the statement [I], for example, recall that the parametrization of the irreducible curve $V(f)$ defined by an analytic function $f$ in $\mathbb{C}\{y, z\}$ has been represented as follows:

$$
V(f):=\left\{\begin{array}{l}
y=t^{n} \\
z=\sum_{i=1}^{r+1} a_{i} t^{\alpha_{i}}\left(1+D_{i}(t)\right)
\end{array}\right.
$$


where $V(f)$ satisfies the same properties and notations as in (1a), (1b), (1c), (1d) and (1e) of (5.1.1), as we have seen in the assumption of this theorem.

Since $2 \leq n<\alpha_{1}$ and $n>\operatorname{gcd}\left(n, \alpha_{1}\right)$ by (1a) and (1b) of (5.1.1), then there is a positive integer $\sigma$ such that $n \sigma<\alpha_{1}<n(\sigma+1)$. Also, in this case it is clear that the standard Puiseux expansion of $V(f)$ can be defined by $y=t^{n}$ and $z=$ $t^{\alpha_{1}}+t^{\alpha_{2}}+\cdots+t^{\alpha_{r+1}}$.

For the converse, it is enough to show by induction on the multiplicity $n$ of the curve $V(f)$ that the multiplicity and Puiseux exponents for the curve $V(f)$ determine uniquely the multiplicity sequence for such an irreducible plane curve singularity.

Suppose that the multiplicity and the Puiseux exponents of $V(f)$ are $\left\{2, \alpha_{1}\right\}$ where $2<\alpha_{1}$ and $\operatorname{gcd}\left(2, \alpha_{1}\right)=1$. Let $\sigma$ be the positive integer such that $n \sigma<\alpha_{1}<$ $n(\sigma+1)$, where $n=2$ and $\alpha_{1}-2 \sigma=1$.

Then, as we have done in (5.1.13), (5.1.14), (5.1.15) and (5.1.16), we have the followings:

$$
V(f): \approx\left\{\begin{array}{l}
y=t^{2} \\
z=t^{\alpha_{1}}
\end{array}\right.
$$

and

$$
V^{(\sigma)}(f): \approx\left\{\begin{array}{l}
v_{\sigma}=t^{2} \\
u_{\sigma}=t^{\alpha_{1}-2 \sigma}
\end{array}\right.
$$

where $\operatorname{gcd}\left(2, \alpha_{1}\right)=1$ and $\alpha_{1}-2 \sigma=1$.

Thus, it is clear that the multiplicity sequence is uniquely determined by the sequence $S=\{2,2, \ldots, 2,1,1\}$, where a counting number $\sigma$ of an element $2 \in S$ is $\frac{\alpha_{1}-1}{2}$ and a counting number of an element $1 \in S$ is two. Thus, if $n=2$, then the proof is easily done.

Now, suppose by the induction proof that if multiplicity of $V(f)$ is either less than a positive integer $n$ or equal to two, then the converse of theorem is true. Assuming that $f$ has a multiplicity either $n$ or at least three at the origin and that the Puiseux expansion of $V(f)$ is defined by the local defining equation in (5.1.1) of the assumption of this theorem where $V(f)$ satisfies the same properties (1a), (1b), (1c), (1d) and (1e) of (5.1.1), then it is enough to show that the multiplicity and Puiseux exponents for the curve $V(f)$ determine uniquely the multiplicity sequence in the standard resolution process of the singularity of $V(f)$.

First, for the convenience of the proof, we may start with assuming that $V(f)$ and $V(g)$ have the same multiplicity and Puiseux exponents, and then it suffices to show that $V(f)$ and $V(g)$ have the same multiplicity sequence.

As compared with the parametrization of $V(f)$ in (5.1.37), for brevity of notation, let the parametrization of $V(g)$ be the Puiseux expansion with the multiplicity and Puiseux exponents $\left\{m, \beta_{1} . \beta_{2}, \ldots, \beta_{u+1}\right\}$, defined by

$$
V(g):=\left\{\begin{array}{l}
y=t^{m} \\
z=\sum_{i=1}^{u+1} b_{i} t^{\beta_{i}}\left(1+L_{i}(t)\right)
\end{array}\right.
$$

where $V(g)$ satisfies the same properties and notations as in (2a), (2b), (2c), (2d) and (2e) of (5.1.2). 
Since $V(f)$ and $V(g)$ have the same multiplicity and Puiseux exponents by assumption, then we have the following:

$$
n=m, r+1=u+1, \text { and } \alpha_{i}=\beta_{i} \text { for } 1 \leq i \leq r+1, \text { with } n>\operatorname{gcd}\left(n, \alpha_{1}\right) .
$$

Also, there is a positive integer $\sigma$ such that $n \sigma<\alpha_{1}<n(\sigma+1), n=m, \alpha_{1}=\beta_{1}$.

In preparation for applying the induction assumption to the proof, first it can be easily shown by Theorem 3.4 with (5.1.19) and (5.1.20), and by (5.1.21) and (5.1.22) that the local defining equations with a new parameter $s$ for $V^{(\sigma)}(f)$ and $V^{(\sigma)}(g)$ are analytically written, respectively as follows: Note that $\alpha_{1}=\beta_{1}$.

$$
\begin{aligned}
& V^{(\sigma)}(f): \approx\left\{\begin{array}{l}
v_{\sigma}=\sum_{i=1}^{r+1} \zeta_{i} s^{n+\alpha_{i}-\alpha_{1}}\left(1+\bar{D}_{i}(s)\right) \\
u_{\sigma}=s^{\alpha_{1}-\sigma n}
\end{array}\right. \\
& V^{(\sigma)}(g): \approx\left\{\begin{array}{l}
v_{\sigma}=\sum_{i=1}^{u+1} \eta_{i} s^{n+\beta_{i}-\beta_{1}}\left(1+\bar{L}_{i}(s)\right) \\
u_{\sigma}=s^{\beta_{1}-\sigma n}
\end{array}\right.
\end{aligned}
$$

where the local defining equation of (5.1.41) satisfies the same properties and notations as in (5.1.21) and (5.1.23), and the local defining equation of (5.1.42) satisfies the same properties and notations as in (5.1.22) and (5.1.24).

If $\alpha_{1}-\sigma n=1$, in this case it is trivial that the same multiplicity sequence is uniquely determined for both $V(f)$ and $V(g)$ as we have seen in (5.1.41) and (5.1.42), because $\beta_{1}=\alpha_{1}$ and $n \geq 2$, and also $\operatorname{gcd}\left(n, \alpha_{1}\right)=\operatorname{gcd}\left(n, \alpha_{1}-\sigma n\right)=\operatorname{gcd}(n, 1)=1$ implies that there are no more exponents $\alpha_{i}$ of the parameter $t$ for $i \geq 2$ in the parametrization for the curve $V(f)$. So, we may start with assuming that $1<\alpha_{1}-$ $\sigma n<n$.

Now, consider two cases: Note that $\alpha_{1}-\sigma n \geq 2$.

(i) $\left(\alpha_{1}-\sigma n\right) \not h n$ and (ii) $\left(\alpha_{1}-\sigma n\right) \mid n$.

Case(i) If $\left(\alpha_{1}-\sigma n\right) \wedge n$, then $V^{(\sigma)}(f)$ and $V^{(\sigma)}(g)$ have the same multiplicity and Puiseux exponents by (5.1.40), (5.1.41) and (5.1.42), that is, the same set $\left\{\alpha_{1}-\sigma n, n, n+\alpha_{2}-\alpha_{1}, n+\alpha_{3}-\alpha_{1}, \ldots, n+\alpha_{r+1}-\alpha_{1}\right\}$, because the local defining equations for both $V^{(\sigma)}(f)$ and $V^{(\sigma)}(g)$ have the Puiseux expansion, satisfying the same kind of properties as we have seen in Lemma 3.3. Since the multiplicity of $V^{(\sigma)}(f)$ at $\left(v_{\sigma}, u_{\sigma}\right)=(0,0)$ is less than $n$, then $V^{(\sigma)}(f)$ and $V^{(\sigma)}(g)$ have the same multiplicity sequence by the induction assumption, and so $V(f)$ and $V(g)$ have the same multiplicity sequence, too.

Case(ii) If $\left(\alpha_{1}-\sigma n\right) \mid n$, then consider the local defining equations with a new parameter $s$ for $V^{(\sigma)}(f)$ and $V^{(\sigma)}(g)$, which are defined by (5.1.40), (5.1.41), and (5.1.42). Since $\alpha_{1}-\sigma n$ is a divisor of $n$, apply the same kind of the method as we have done in the proof of the sufficiency of the Case(B), to the local defining equation analytically equivalent to that of $V^{(\sigma)}(f)$ in (5.1.41) and (5.1.42), and then we can 
easily get that $V^{(\sigma)}(f)$ and $V^{(\sigma)}(g)$ are analytically well-defined by

$$
\begin{aligned}
& V^{(\sigma)}(f): \approx\left\{\begin{array}{l}
v_{\sigma}^{\prime}=\sum_{i=2}^{r+1} \zeta_{i} s^{n+\alpha_{i}-\alpha_{1}}\left(1+\bar{D}_{i}(s)\right) \\
u_{\sigma}^{\prime}=s^{\alpha_{1}-\sigma n} .
\end{array}\right. \\
& V^{(\sigma)}(g): \approx\left\{\begin{array}{l}
v_{\sigma}^{\prime \prime}=\sum_{i=2}^{u+1} \eta_{i} s^{n+\beta_{i}-\beta_{1}}\left(1+\bar{L}_{1}(s)\right) \\
u_{\sigma}^{\prime \prime}=s^{\beta_{1}-\sigma n} .
\end{array}\right.
\end{aligned}
$$

That is, $V^{(\sigma)}(f)$ of (5.1.43) satisfies the same kind of assumptions relative to the local coordinates $\left(v_{\sigma}^{\prime}, u_{\sigma}^{\prime}\right)$ as $V^{(\sigma)}(f)$ of (5.1.21) has done relative to the local coordinates $\left(v_{\sigma}, u_{\sigma}\right)$. Also, $V^{(\sigma)}(g)$ of (5.1.44) satisfies the same kind of assumptions relative to the local coordinates $\left(v_{\sigma}^{\prime \prime}, u_{\sigma}^{\prime \prime}\right)$ as $V^{(\sigma)}(g)$ of (5.1.22) has done relative to the local coordinates $\left(v_{\sigma}, u_{\sigma}\right)$. Since $\alpha_{1}-\sigma n<n+\alpha_{2}-\alpha_{1}$ and $\alpha_{1}-\sigma n>\operatorname{gcd}\left(\alpha_{1}-\sigma n, n+\right.$ $\left.\alpha_{2}-\alpha_{1}\right)$, then $V^{(\sigma)}(f)$ and $V^{(\sigma)}(g)$ have the same multiplicity and Puiseux exponents by (5.1.40), (5.1.41) and (5.1.42), that is, the same set $\left\{\alpha_{1}-\sigma n, n+\alpha_{2}-\alpha_{1}, n+\alpha_{3}-\right.$ $\left.\alpha_{1}, \ldots, n+\alpha_{r+1}-\alpha_{1}\right\}$, without containing an element $n$. Since the multiplicity of $V^{(\sigma)}(f)$ at $\left(v_{\sigma}, u_{\sigma}\right)=(0,0)$ is less than $n$, then $V^{(\sigma)}(f)$ and $V^{(\sigma)}(g)$ have the same multiplicity sequence by the induction assumption, and so $V(f)$ and $V(g)$ have the same multiplicity sequence, too.

(II) By Theorem 3.4, the local defining equation for $V(h)$ can be analytically written as follows:

$$
V(h): \approx\left\{\begin{aligned}
y & =s^{l_{1}} \\
z & =\sum_{i=1}^{v+1} \zeta_{i} s^{\gamma+l_{i}-l_{1}}\left(1+\bar{R}_{i}(s)\right)
\end{aligned}\right.
$$

where

(i) the $\zeta_{i}$ are all nonzero numbers for $i=1,2, \ldots, v+1$,

(ii) for $i=1,2, \ldots, v+1$, define $\bar{R}_{i}(s)$ by

$$
\begin{gathered}
\bar{R}_{1}(s)=\sum_{i=1}^{\varepsilon_{1}} \bar{c}_{1 i} s^{i \tau_{1}} \in \mathbb{C}[s], \\
\bar{R}_{2}(s)=\sum_{i=1}^{\varepsilon_{2}} \bar{c}_{2 i} s^{i \tau_{2}} \in \mathbb{C}[s], \\
\cdots \cdots \\
\bar{R}_{v}(s)=\sum_{i=1}^{\varepsilon_{v}} \bar{c}_{v i} s^{i \tau_{v}} \in \mathbb{C}[s], \\
\bar{R}_{v+1}(s)=\sum_{i=1}^{\infty} \bar{c}_{v+1, i} s^{i} \in \mathbb{C}\{s\},
\end{gathered}
$$

such that all $\bar{c}_{j, i(j)}$ are complex numbers with $1 \leq j \leq v+1$ and $1 \leq i(j) \leq \varepsilon_{j}$, some of which may be zero. 
For brevity of notation in (5.1.45), write

$$
\omega=l_{1} \text { and } \delta_{i}=\gamma+l_{i}-l_{1} \text { for } 1 \leq i \leq v+1 .
$$

Note by (3a) of (5.1.3) that $2 \leq l_{1}<\gamma=\delta_{1}$ and $\delta_{i+1}-\delta_{i}=l_{i+1}-l_{i}>0$ for $1 \leq i \leq v+1$.

Then, we claim the following properties:

(i) $2 \leq \omega<\delta_{1}<\delta_{2}<\delta_{3}<\cdots<\delta_{v+1}$.

(ii) $\omega \geq \operatorname{gcd}\left(\omega, \delta_{1}\right)>\operatorname{gcd}\left(\omega, \delta_{1}, \delta_{2}\right)>\cdots>\operatorname{gcd}\left(\omega, \delta_{1}, \delta_{2}, \ldots, \delta_{v+1}\right)=1$.

Firstly, to prove (i) is clear, because such inequalities have been already defined by (3a) of (5.1.3), with an additional notation $\omega=l_{1}$. Secondly, to prove (ii), note by the properties $(3 \mathrm{~b})$ and $(3 \mathrm{~d})$ in (5.1.3) and by (5.1.47) that

$$
\begin{aligned}
& \operatorname{gcd}\left(\omega, \delta_{1}\right)=\operatorname{gcd}\left(l_{1}, \gamma\right)=\operatorname{gcd}\left(\gamma, l_{1}\right)=\tau_{1}, \\
& \operatorname{gcd}\left(\omega, \delta_{1}, \delta_{2}\right)=\operatorname{gcd}\left(\gamma, l_{1}, \gamma+l_{2}-l_{1}\right)=\operatorname{gcd}\left(\gamma, l_{1}, l_{2}\right)=\tau_{2}, \\
& \operatorname{gcd}\left(\omega, \delta_{1}, \delta_{2}, \delta_{3}\right)=\operatorname{gcd}\left(\omega, \delta_{1}, \delta_{2}, \gamma+l_{3}-l_{1}\right)=\operatorname{gcd}\left(\gamma, l_{1}, l_{2}, \gamma+l_{3}-l_{1}\right)= \\
& \operatorname{gcd}\left(\gamma, l_{1}, l_{2}, l_{3}\right)=\tau_{3}, \\
& \cdots \cdots \\
& \operatorname{gcd}\left(\omega, \delta_{1}, \delta_{2}, \ldots, \delta_{v+1}\right)=\operatorname{gcd}\left(\omega, \delta_{1}, \delta_{2}, \ldots, \delta_{v}, \gamma+l_{v+1}-l_{1}\right)= \\
& \operatorname{gcd}\left(\gamma, l_{1}, l_{2}, \ldots, l_{v}, \gamma+l_{v+1}-l_{1}\right)=\tau_{v+1} \quad \text { and } \\
& \tau_{1}<\tau_{2}<\tau_{3}<\cdots<\tau_{v+1}=1 .
\end{aligned}
$$

Thus, the proofs of (i) and (ii) of the claim are just finished. Now, since $V(h)$, which is defined by (5.1.45) and (5.1.47), satisfies the same kind of properties as $V(f)$ of either Lemma 4.3 or (I) of this theorem does, then it is enough to consider two cases: (IIa) $l_{1}>\operatorname{gcd}\left(l_{1}, \gamma\right)$ and (IIb) $l_{1}$ is a divisor of $\gamma$.

(IIa) Let $l_{1}>\operatorname{gcd}\left(l_{1}, \gamma\right)$. By applying (5.1.45) with an additional proof of the claim to (I), then we get the following:

$$
\begin{aligned}
& V(f) \equiv V(h) \quad \text { (multi. seq.) } \\
\Longleftrightarrow & n=l_{1}, \quad r+1=v+1 \quad \text { and } \quad \alpha_{i}=\gamma+l_{i}-l_{1} \quad \text { for } \quad 1 \leq i \leq r+1,
\end{aligned}
$$

which is equivalent to the fact that the Puiseux pairs for both $V(f)$ and $V(h)$ are the same, by Definition 4.4 .

Note that $\alpha_{i}=\gamma+l_{i}-l_{1}$ is the same as $l_{i}=n+\alpha_{i}-\alpha_{1}$, because $\alpha_{1}=\gamma$ and $\ell_{1}=n$. Thus, the proof of (IIa) is done.

(IIb) Let $l_{1}$ be a divisor of $\gamma$. Since $\gamma$ is a multiple of $l_{1}$, then apply the same kind of the method as we have used in the local defining equations of either (5.1.32) or (5.1.34), to the local defining equation in (5.1.45).

Then, $V(h)$ has the set of multiplicity and Puiseux exponents, that is, $\left\{\ell_{1}, \gamma+\right.$ $\left.\ell_{2}-\ell_{1}, \ldots, \gamma+\ell_{v+1}-\ell_{1}\right\}$, without containing an element $\gamma$.

So, by the similar way as we have used in (IIa), we can easily prove the following:

$$
V(f) \equiv V(h) \quad \text { (multi. seq.) }
$$

$\Longleftrightarrow n=l_{1}, \alpha_{1}=\gamma+l_{2}-l_{1}, r+1=v \quad$ and $\quad \alpha_{i}=\gamma+l_{i+1}-l_{1} \quad$ for $\quad 2 \leq i \leq r+1$, 
which is equivalent to the fact that the Puiseux pairs for both $V(f)$ and $V(h)$ are the same, by Definition 4.4.

Thus, this completes the proof of Theorem 5.1.

Moreover, the proof of Corollary 5.2 follows just from Theorem 4.5 and Theorem 5.1 .

EXAMPLE: Let $V=\{(y, z): f(y, z)=0\}$ and $W_{\lambda}=\left\{(y, z): g_{\lambda}(y, z)=0\right\}$ be analytic varieties at $(y, z)=(0,0)$, respectively, satisfying the following the properties:

(i) $f(y, z)=0 \Longleftrightarrow y=t^{4}, z=t^{6}+t^{9}$.

(ii) $g_{\lambda}(y, z)=0 \Longleftrightarrow y=t^{4}+\lambda t^{7}, z=t^{6}+t^{9}$ for arbitrary complex number $\lambda$.

Now, the problem is to find when $f$ and $g_{\lambda}$ have the same topological type of singularity at the origin. To solve it, since $f(y, z)$ is analytically irreducible at $(y, z)=$ $(0,0)$, by Theorem 3.4 let $s$ be a new parameter defined by $s=t\left(1+t^{3}\right)^{\frac{1}{6}}$ for the equivalent parametrization of $V$ defined by the equation $f(y, z)=0$. By Theorem 3.4, $t=s\left\{1+s^{3}\left(\frac{1}{-6}+\sum_{k>1} a_{k} s^{k}\right)\right\}$ where all the $a_{k}$ are complex numbers, and so the equivalent parametrization for $W_{\lambda}$ have the defining equation with the parameter $s$ as follows:

$$
z(s)=s^{6} \quad \text { and } \quad y(s)=s^{4}+s^{7}\left\{\left(\frac{4}{-6}+\lambda\right)+\sum_{k \geq 1} b_{k} s^{k}\right\}
$$

where all the $b_{k}$ are complex numbers. Therefore, $\bar{V}$ and $W_{\lambda}$ have the same topological type of the singularity at the origin if and only if $\lambda \neq \frac{2}{3}$.

6. The proof of the Inverse Theorem (The relationship between Puiseux pairs and the reverse Puiseux pairs) by an equivalence of irreducible parametrization. In this section, we prove analytically the Inverse Theorem (Theorem 6.2). Before proving it, note by ([Ab1]) that the Inverse Theorem (the relationship between Puiseux pairs and the reverse Puiseux pairs), which was written in the algebraic statement, was already proved. Note by ([Ab2]) that the equivalence of the Puiseux expansion with the multiplicity and Puiseux exponents and the multiplicity sequences for irreducible plane curve singularities was once proved, too. The Inverse Theorem (Theorem 6.2) has been analytically written in ([Ab3]) without any other proof, which is the restatement of the inverse Theorem ([Ab1]). Now, we may assume begin with the definition of the reverse Puiseux pairs. Then by Theorem 3.4, we prove very easily this theorem, without using any other lemma.

DEFINITION 6.1. Let the parametrization for the curve $C$ be given as follows:

$$
\begin{aligned}
x=t^{u} & \\
y=\eta(t)= & \cdots+b_{m_{1}} t^{m_{1}}+\cdots+b_{m_{2}} t^{m_{2}}+\cdots \\
& +b_{m_{3}} t^{m_{3}}+\cdots+b_{m_{h}} t^{m_{h}}+\cdots
\end{aligned}
$$

where in the expansion, $u$ is the multiplicity of the curve $C$ at $(x, y)=(0,0), m_{1}$ is the smallest exponent not divisible by $u$ whose coefficient $b_{m_{1}}$ is nonzero, $m_{2}$ is the smallest exponent not divisible by $\operatorname{gcd}\left(u, m_{1}\right)$ whose coefficient $b_{m_{2}}$ is nonzero, $m_{3}$ is the smallest exponent not divisible by $\operatorname{gcd}\left(u, m_{1}, m_{2}\right)$ whose coefficient $b_{m_{3}}$ is nonzero,..., and $m_{h}$ is the smallest exponent for which $\operatorname{gcd}\left(u, m_{1}, \ldots, m_{h}\right)=1$ whose coefficient $b_{m_{h}}$ is nonzero. Let $m_{0}=u$ and $d_{i+1}=\operatorname{gcd}\left(m_{0}, m_{1}, \ldots, m_{i}\right)$ for $0 \leq i \leq h$. Then, $d_{1}>d_{2}>\cdots>d_{h+1}=1$. We define the Puiseux pairs $\left(\lambda_{1}, \mu_{1}\right),\left(\lambda_{2}, \mu_{2}\right), \ldots,\left(\lambda_{h}, \mu_{h}\right)$ 
of the curve $C$ at $(y, z)=(0,0)$ by putting

$$
\lambda_{i}=\frac{m_{i}}{d_{i+1}} \text { and } \mu_{i}=\frac{d_{i}}{d_{i+1}} \text { for } 1 \leq i \leq h .
$$

Now, to state the Inversion Theorem, define the reverse Puiseux pairs, that is, $\left(\lambda_{1}^{\prime}, \mu_{1}^{\prime}\right)$, $\left(\lambda_{2}^{\prime}, \mu_{2}^{\prime}\right), \ldots,\left(\lambda_{h}^{\prime}, \mu_{h}^{\prime}\right)$ of the curve $C$ at $(y, x)=(0,0)$ by expanding $x$ in terms of $y$ thus. Let $u^{\prime}$ be the order of zero in $\eta(t)$ at $t=0$. Note that $u^{\prime}$ is a positive integer with $u^{\prime} \leq m_{1}$. follows:

By Newton's Theorem, the parametrization for the curve $C$ can be given as

$$
\begin{aligned}
y=t^{u^{\prime}} & \\
x=\xi(t)= & \cdots+c_{m_{1}^{\prime}} t^{m_{1}^{\prime}}+\cdots+c_{m_{2}^{\prime}} t^{m_{2}^{\prime}}+\cdots \\
& +c_{m_{3}^{\prime}} t^{m_{3}^{\prime}}+\cdots+c_{m_{h}^{\prime}} t^{m_{h}^{\prime}}+\cdots
\end{aligned}
$$

where in the expansion, $m_{1}^{\prime}$ is the smallest exponent not divisible by $u^{\prime}$ whose coefficient $c_{m_{1}^{\prime}}$ is nonzero, $m_{2}^{\prime}$ is the smallest exponent not divisible by $\operatorname{gcd}\left(u^{\prime}, m_{1}^{\prime}\right)$ whose coefficient $c_{m_{2}^{\prime}}$ is nonzero, $m_{3}^{\prime}$ is the smallest exponent not divisible by $\operatorname{gcd}\left(u^{\prime}, m_{1}^{\prime}, m_{2}^{\prime}\right)$ whose coefficient $c_{m_{3}^{\prime}}$ is nonzero,..., and $m_{k}^{\prime}$ is the smallest exponent for which $\operatorname{gcd}\left(u^{\prime}, m_{1}^{\prime}, \ldots, m_{h}^{\prime}\right)=1$ whose coefficient $c_{m_{h^{\prime}}^{\prime}}$ is nonzero. Let $m_{0}^{\prime}=u$, and $d_{i+1}^{\prime}=$ $\operatorname{gcd}\left(m_{0}^{\prime}, m_{1}^{\prime}, \ldots, m_{i}^{\prime}\right)$ for $0 \leq i \leq h$. Then, $d_{1}^{\prime}>d_{2}^{\prime}>\cdots>d_{h+1}^{\prime}=1$. We define the reverse Puiseux pairs $\left(\lambda_{1}^{\prime}, \mu_{1}^{\prime}\right),\left(\lambda_{2}^{\prime}, \mu_{2}^{\prime}\right), \ldots,\left(\lambda_{h^{\prime}}^{\prime}, \mu_{h^{\prime}}^{\prime}\right)$ of the curve $C$ at $(y, x)=(0,0)$ by putting

$$
\lambda_{i}^{\prime}=\frac{m_{i}^{\prime}}{d_{i+1}^{\prime}} \text { and } \mu_{i}^{\prime}=\frac{d_{i}^{\prime}}{d_{i+1}^{\prime}} \text { for } 1 \leq i \leq h^{\prime}
$$

TheOrEm 6.2 (InVERsion TheOREM). We use the same properties and notations as we have seen in Definition 6.1. The relationship between the Puiseux pairs and the reverse Puiseux pairs is given by the following: Note that $u \leq u^{\prime} \leq m_{1}$.

(1) If $u^{\prime}=u$, then $h=h, \lambda_{i}^{\prime}=\lambda_{i}$ for $1 \leq i \leq h$, and $\mu_{i}^{\prime}=\mu_{i}$ for $1 \leq i \leq h$.

(2) If $u^{\prime}=m_{1}$, then $h^{\prime}=h, \lambda_{1}^{\prime}=\mu_{1}, \mu_{1}^{\prime}=\lambda_{1}, \lambda_{i}^{\prime}=\lambda_{i}-\left(\lambda_{1}-\mu_{1}\right) \mu_{2} \mu_{3} \cdots \mu_{i}$ for $2 \leq i \leq h$, and $\mu_{i}^{\prime}=\mu_{i}$ for $2 \leq i \leq h$.

(3) If $u \neq u^{\prime} \neq m_{1}$, then $h^{\prime}=h+1, \lambda_{1}^{\prime}=1, \mu_{1}^{\prime}=\frac{u^{\prime}}{u}, \lambda_{i+1}^{\prime}=\lambda_{1}-\frac{u^{\prime}}{u-1} \mu_{1} \mu_{2} \cdots \mu_{i}$ for $1 \leq i \leq h$, and $\mu_{i+1}^{\prime}=\mu_{i}$ for $1 \leq i \leq h$.

Proof of Theorem. It is enough to prove (1), (2), and (3), respectively as follows: (1) Let $u^{\prime}=u$. For brevity, write $u^{\prime}=m_{0}$. Then by Theorem 3.4, the parametrization of $C$ can be easily rearranged as follows:

$$
\begin{aligned}
x= & t^{u} \quad \text { and } \\
y= & c_{1} t^{m_{0}}\left\{\left(1+D_{0}(t)\right)+a_{1} t^{m_{1}-m_{0}}\left(1+D_{1}(t)\right)+\cdots\right. \\
& \left.+a_{h-1} t^{m_{h-1}-m_{0}}\left(1+D_{h-1}(t)\right)+a_{h} t^{m_{h}-m_{0}}\left(1+D_{h}(t)\right)\right\} \\
= & c_{1} t^{m_{0}}\{1+H(t)\}
\end{aligned}
$$

where (i) $2 \leq u$ and $2 \leq m_{0}<m_{1}<m_{2}<\cdots<m_{h}$, (ii) $u=m_{0}=d_{1}>d_{2}>$ $\cdots>d_{h+1}=1$ with $\operatorname{gcd}\left(m_{0}, m_{1}, \ldots, m_{i}\right)=d_{i+1}$ for $0 \leq i \leq h$, (iii) $p_{1}, p_{2}, \ldots$, 
$p_{h}$ are nonnegative integers such that $p_{i+1} d_{i+1}<m_{i+1}-m_{i}<\left(p_{i+1}+1\right) d_{i+1}$ for $0 \leq i \leq h-1$, (iv) $D_{j}(t)=\sum_{i=1}^{p_{j+1}} c_{j+1, i} t^{i d_{j+1}} \in \mathbb{C}[t]$, the ring of polynomials in $t$, for $0 \leq j \leq h-1$ and $D_{h}(t)=\sum_{i=1}^{\infty} c_{h+1, i} t^{i} \in \mathbb{C}\{t\}$, the ring of convergent power series at the origin in $\mathbb{C}^{2},(\mathrm{v})$ the $a_{i}$ are nonzero complex numbers for $i=1,2, \ldots, h$ from the above parametrization of the curve $C$.

Let $s$ be the new parameter defined by a conformal mapping of one complex variable $t$ at the origin such that $s(t)=c_{1}^{\frac{1}{m_{0}}} t(1+H(t))^{\frac{1}{m_{0}}}$ with $y=s^{m_{0}}$ and $s(0)=$ 0 . Then by Theorem 3.4, an equivalence of the given parametrization of $C$ can be represented as follows:

$$
\begin{aligned}
y= & s^{m_{0}} \text { and } \\
x= & c_{1}^{-\frac{u}{m_{0}}} s^{u}\left\{\left(1+Q_{0}^{*}(s)\right)+s^{m_{1}-m_{0}}\left(b_{10}+Q_{1}^{*}(s)\right)+\cdots\right. \\
& \left.\left.+s^{m_{h-1}-m_{0}}\left(b_{h-1,0}+Q_{h-1}^{*}(s)\right)\right\}+s^{m_{h}-m_{0}}\left(b_{h, 0}+Q_{h}^{*}(s)\right)\right\} \\
= & c_{1}{ }^{-\frac{u}{m_{0}}} s^{u}\{1+L(s)\},
\end{aligned}
$$

where (i) $Q_{j}^{*}(s)=\sum_{i=1}^{p_{j+1}} b_{j+1, i} s^{i d_{j+1}} \in \mathbb{C}[s]$ for $0 \leq j \leq h-1$ and $Q_{h}^{*}(s)=$ $\sum_{i=1}^{\infty} b_{h+1, i} s^{i} \in \mathbb{C}\{s\}$, (ii) all the $b_{j i}$ are complex numbers and the $b_{i 0}$ are nonzero for $1 \leq i \leq h$, (iii) $L(s)$ is just the substitution. Since $u^{\prime}=m_{0}=u$ and $m_{i}^{\prime}=m_{i}$ for $1 \leq i \leq h=h^{\prime}$, by definition of the reverse Puiseux pairs, there is nothing to prove.

(2) Let $u^{\prime}=m_{1}$. Then by Theorem 3.4, the parametrization of $C$ can be easily rearranged as follows:

$$
\begin{aligned}
x= & t^{u} \quad \text { and } \\
y= & c_{1} t^{m_{1}}\left\{\left(1+D_{1}(t)\right)+a_{1} t^{m_{2}-m_{1}}\left(1+D_{2}(t)\right)+\cdots\right. \\
& \left.+a_{h-1} t^{m_{h-1}-m_{1}}\left(1+D_{h-1}(t)\right)+a_{h} t^{m_{h}-m_{1}}\left(1+D_{h}(t)\right)\right\} \\
= & c_{1} t^{m_{1}}\{1+H(t)\},
\end{aligned}
$$

where (i) $2 \leq u$ and $2 \leq m_{1}<m_{2}<\cdots<m_{h}$, (ii) $u=d_{1}>d_{2}>\cdots>d_{h+1}=1$ with $\operatorname{gcd}\left(u, m_{1}, \ldots, m_{i}\right)=d_{i+1}$ for $0 \leq i \leq h$, (iii) $p_{1}, p_{2}, \ldots, p_{h}$ are nonnegative integers such that $p_{i+1} d_{i+1}<m_{i+1}-m_{i}<\left(p_{i+1}+1\right) d_{i+1}$ for $0 \leq i \leq h-1$, (iv) $D_{j}(t)=\sum_{i=1}^{p_{j+1}} c_{j+1, i} t^{i d_{j+1}} \in \mathbb{C}[t]$, the ring of polynomials in $t$, for $0 \leq j \leq h-1$ and $D_{h}(t)=\sum_{i=1}^{\infty} c_{h, i} t^{i} \in \mathbb{C}\{t\}$, the ring of convergent power series at the origin in $\mathbb{C}^{2},(\mathrm{v})$ the $a_{i}$ are nonzero complex numbers for $i=1,2, \ldots, h$ from the above parametrization of the curve $C$.

Let $s$ be the new parameter defined by a conformal mapping of one complex variable $t$ at the origin such that $s(t)=c_{1}^{\frac{1}{m_{1}}} t(1+H(t))^{\frac{1}{m_{1}}}$ with $y=s^{m_{1}}$ and $s(0)=$ 0 . Then by Theorem 3.4, an equivalence of the given parametrization of $C$ can be represented as follows:

$$
\begin{aligned}
y= & s^{m_{1}} \quad \text { and } \\
x= & c_{1}^{-\frac{u}{m_{1}}} s^{u}\left\{\left(1+Q_{1}^{*}(s)\right)+s^{m_{2}-m_{1}}\left(b_{2,0}+Q_{2}^{*}(s)\right)+\cdots\right. \\
& \left.\left.+s^{m_{h-1}-m_{1}}\left(b_{h-1,0}+Q_{h-1}^{*}(s)\right)\right\}+s^{m_{h}-m_{1}}\left(b_{h, 0}+Q_{h}^{*}(s)\right)\right\} \\
= & c_{1}^{-\frac{u}{m_{1}}} s^{u}\{1+L(s)\},
\end{aligned}
$$


where (i) $Q_{j}^{*}(s)=\sum_{i=1}^{p_{j+1}} b_{j+1, i} s^{i d_{j+1}} \in \mathbb{C}[s]$ for $0 \leq j \leq h-1$ and $Q_{h}^{*}(s)=$ $\sum_{i=1}^{\infty} b_{h, i} s^{i} \in \mathbb{C}\{s\}$, (ii) all the $b_{j i}$ are complex numbers and the $b_{i 0}$ are nonzero for $1 \leq i \leq h$, (iii) $L(s)$ is just the substitution.

To find the reverse Puiseux pairs, by (6.1.1), (6.1.3), (6.2.3) and (6.2.4), observe the following:

$$
\begin{aligned}
& u^{\prime}=m_{1}, m_{1}^{\prime}=u, m_{2}^{\prime}=u+m_{2}-m_{1}, \ldots, \\
& m_{h}^{\prime}=u+m_{h}-m_{1}, d_{1}^{\prime}=m_{1}=u^{\prime} .
\end{aligned}
$$

By definition, recall that $d_{i+1}^{\prime}=\operatorname{gcd}\left(u^{\prime}, m_{1}^{\prime}, m_{2}^{\prime}, \ldots, m_{i}^{\prime}\right)$ for $1 \leq i \leq h$.

So, by (6.2.5) and the definition of $d_{i}, d_{i+1}^{\prime}=\operatorname{gcd}\left(m_{1}, u, u+m_{2}-m_{1}, u+m_{3}-\right.$ $\left.m_{1}, \ldots, u+m_{i}-m_{1}\right)=\operatorname{gcd}\left(u, m_{1}, m_{2}, \ldots, m_{i}\right)=d_{i+1}$ for $1 \leq i \leq h$.

Now, by definition of the reverse Puiseux pairs, the proof of (2) can be done from the following facts $(2 \mathrm{a}),(2 \mathrm{~b}),(2 \mathrm{c}),(2 \mathrm{~d})$ and $(2 \mathrm{e})$ :

(2a) Then, it is trivial that $h=h^{\prime}$.

(2b) Then, $\lambda_{1}^{\prime}=\frac{m_{1}^{\prime}}{d_{2}^{\prime}}=\frac{u}{d_{2}^{\prime}}$ and $\mu_{1}=\frac{d_{1}}{d_{2}}=\frac{u}{d_{2}}$. Since $d_{2}^{\prime}=\operatorname{gcd}\left(m_{1}, u\right)$ and $d_{2}=\operatorname{gcd}\left(u, m_{1}\right)$, then the proof is done.

(2c) Note that $\mu_{1}^{\prime}=\frac{d_{1}^{\prime}}{d_{2}^{\prime}}=\frac{m_{1}}{\operatorname{gcd}\left(m_{1}, u\right)}=\lambda_{1}$.

(2d) By definition and (6.2.5), $\lambda_{i}^{\prime}=\frac{m_{i}^{\prime}}{d_{i+1}^{\prime}}=\frac{u+m_{i}-m_{1}}{d_{i+1}^{\prime}}$.

Also, $\lambda_{i}-\left(\lambda_{1}-\mu_{1}\right) \mu_{2} \mu_{3} \cdots \mu_{i}=\frac{m_{i}}{d_{i+1}}-\left(\frac{m_{1}}{d_{2}}-\frac{d_{1}}{d_{2}}\right) \frac{d_{2}}{d_{i+1}}=\frac{m_{i}-\left(m_{1}-d_{1}\right)}{d_{i+1}}$. Since $d_{1}=u$ and $d_{i}^{\prime}=d_{i}$ for $2 \leq i \leq h+1$, then the proof is done.

(2e) Note by definition that $\mu_{i}^{\prime}=\frac{d_{i}^{\prime}}{d_{i+1}^{\prime}}$ and $\mu_{i}=\frac{d_{i}}{d_{i+1}}$ for $2 \leq i \leq h$. Since $d_{i}^{\prime}=d_{i}$ for $2 \leq i \leq h+1$, then the proof is done. Thus, the proof of (2) is finished.

(3) First, note that $u<u^{\prime}<m_{1}$ and that $u^{\prime}$ is divisible by $u$. Then by Theorem 3.4, the parametrization of $C$ can be easily rearranged as follows: For convenience of notation, write $m_{0}=u^{\prime}$.

$$
\begin{aligned}
x= & t^{u} \quad \text { and } \\
y= & c_{1} t^{m_{0}}\left\{\left(1+D_{0}(t)\right)+a_{1} t^{m_{1}-m_{0}}\left(1+D_{1}(t)\right)+\cdots\right. \\
& \left.+a_{h-1} t^{m_{h-1}-m_{0}}\left(1+D_{h-1}(t)\right)+a_{h} t^{m_{h}-m_{0}}\left(1+D_{h}(t)\right)\right\} \\
= & c_{1} t^{m_{0}}\{1+H(t)\},
\end{aligned}
$$

where (i) $2 \leq u$ and $2 \leq m_{0}=u^{\prime}<m_{1}<m_{2}<\cdots<m_{h}$, (ii) $u=d_{1}>d_{2}>$ $\cdots>d_{h+1}=1$ with $\operatorname{gcd}\left(u, m_{0}, m_{1}, \ldots, m_{i}\right)=d_{i+1}$ for $1 \leq i \leq h$, (iii) $p_{1}, p_{2}, \ldots$, $p_{h}$ are nonnegative integers such that $p_{i+1} d_{i+1}<m_{i+1}-m_{i}<\left(p_{i+1}+1\right) d_{i+1}$ for $0 \leq i \leq h-1$, (iv) $D_{j}(t)=\sum_{i=1}^{p_{j+1}} c_{j+1, i} t^{i d_{j+1}} \in \mathbb{C}[t]$, the ring of polynomials in $t$, for $0 \leq j \leq h-1$ and $D_{h}(t)=\sum_{i=1}^{\infty} c_{j+1, i} t^{i} \in \mathbb{C}\{t\}$, the ring of convergent power series at the origin in $\mathbb{C}^{2},(\mathrm{v})$ the $a_{i}$ are nonzero complex numbers for $i=1,2, \ldots, h$ from the above parametrization of the curve $C$.

Let $s$ be the new parameter defined by a conformal mapping of one complex variable $t$ at the origin such that $s(t)=c_{1}^{\frac{1}{m_{0}}} t(1+H(t))^{\frac{1}{m_{0}}}$ with $y=s^{m_{0}}$ and $s(0)=$ 0 . Then by Theorem 3.4, an equivalence of the given parametrization of $C$ can be 
represented as follows: Note that $m_{0}=u^{\prime}$.

$$
\begin{aligned}
y= & s^{m_{0}} \quad \text { and } \\
x= & c_{1}^{-\frac{u}{m_{0}}} s^{u}\left\{\left(1+Q_{0}^{*}(s)\right)\right. \\
& +s^{m_{1}-m_{0}}\left(b_{1,0}+Q_{1}^{*}(s)\right)+s^{m_{2}-m_{0}}\left(b_{2,0}+Q_{2}^{*}(s)\right)+\cdots \\
& \left.\left.+s^{m_{h-1}-m_{0}}\left(b_{h-1,0}+Q_{h-1}^{*}(s)\right)\right\}+s^{m_{h}-m_{0}}\left(b_{h, 0}+Q_{h}^{*}(s)\right)\right\} \\
= & c_{1}{ }^{-\frac{u}{m_{0}}} s^{u}\{1+L(s)\},
\end{aligned}
$$

where (i) $Q_{j}^{*}(s)=\sum_{i=1}^{p_{j}} b_{j+1, i} s^{i d_{j+1}} \in \mathbb{C}[s]$ for $0 \leq j \leq h-1$ and $Q_{h}^{*}(s)=\sum_{i=1}^{\infty} b_{h, i} s^{i} \in$ $\mathbb{C}\{s\}$, (ii) all the $b_{j i}$ are complex numbers and the $b_{i 0}$ are nonzero for $1 \leq i \leq h$, (iii) $L(s)$ is just the substitution.

Since $u<u^{\prime}=m_{0}<m_{1}$ and $m_{0}$ is divisible by $u$, then by (6.1.1), (6.1.3), (6.2.6) and (6.2.7), observe the followings:

$$
\begin{aligned}
& d_{1}=u, d_{2}=\operatorname{gcd}\left(u, m_{1}\right), d_{i+1}=\operatorname{gcd}\left(u, m_{1}, m_{2}, \ldots, m_{i}\right) \quad \text { for } 2 \leq i \leq h, \\
& u^{\prime}=m_{0}, m_{1}^{\prime}=u, m_{2}^{\prime}=u+m_{1}-m_{0}, \ldots, m_{h}^{\prime}=u+m_{h-1}-m_{0}, m_{h+1}^{\prime}=u+m_{h}-m_{0}, \\
& d_{1}^{\prime}=u^{\prime}=m_{0}, d_{2}^{\prime}=\operatorname{gcd}\left(u^{\prime}, u\right)=u, d_{3}^{\prime}=\operatorname{gcd}\left(u^{\prime}, u, u+m_{1}-u^{\prime}\right)=\operatorname{gcd}\left(u, m_{1}\right), \\
& d_{i+1}^{\prime}=\operatorname{gcd}\left(u^{\prime}, u, u+m_{1}-u^{\prime}, \ldots, u+m_{i-1}-u^{\prime}\right) \\
& \quad=\operatorname{gcd}\left(u, m_{1}, m_{2}, \ldots, m_{i-1}\right) \quad \text { for } 2 \leq i \leq h+1 .
\end{aligned}
$$

Now, by definition of the reverse Puiseux pairs, the proof of (3) can be done from the following facts (3a), (3b), (3c), (3d) and (3e):

(3a) Note that $h$ is the number of distinct elements in the set $\left\{d_{1}>d_{2}>\cdots>\right.$ $\left.d_{h}\right\}$, and that $h^{\prime}$ is the number of distinct elements in the set $\left\{d_{1}^{\prime}>d_{2}^{\prime}>\cdots>d_{h^{\prime}}^{\prime}\right\}$. Then it is clear that $h^{\prime}=h+1$, because $u<u^{\prime}=m_{0}<m_{1}$ and $m_{0}$ is divisible by $u$.

(3b) Then, $\lambda_{1}^{\prime}=\frac{m_{1}^{\prime}}{d_{2}^{\prime}}=\frac{u}{u}=1$.

(3c) Then, $\mu_{1}^{\prime}=\frac{d_{1}^{\prime}}{d_{2}^{\prime}}=\frac{u^{\prime}}{u}$.

(3d) By definition and (6.2.8), $\lambda_{i+1}^{\prime}=\frac{m_{i+1}^{\prime}}{d_{i+2}^{\prime}}=\frac{u+m_{i+1}-m_{1}}{d_{i+2}^{\prime}}$ for $1 \leq i \leq h$. Also, $\lambda_{i}-\left(\frac{u^{\prime}}{u}-1\right) \mu_{1} \mu_{2} \cdots \mu_{i}=\frac{m_{i+1}}{d_{i+1}}-\left(\frac{u^{\prime}}{u}-1\right) \frac{d_{1}}{d_{2}} \frac{d_{2}}{d_{3}} \cdots \frac{d_{i}}{d_{i+1}}=\frac{m_{i+1}}{d_{i+1}}-\left(\frac{m_{1}}{u}-1\right) \frac{d_{1}}{d_{i+1}}$ for $1 \leq i \leq h$. Since $d_{i+2}^{\prime}=d_{i+1}$ and $d_{1}=u$, then the proof is done.

(3e) Then, $\mu_{i+1}^{\prime}=\frac{d_{i+1}^{\prime}}{d_{i+2}^{\prime}}=\frac{d_{i}}{d_{i+1}}=\mu_{i}$ for $1 \leq i \leq h$, and so the proof of (3) is done. Therefore, we have completed the proof of the theorem.

Acknowledgement. The author is very appreciated for Dr. Young Rock Kim's typewriting help.

\section{REFERENCES}

[Ab1] AbHYAnkaR, S. S., Inversion and invariance of characteristic pairs, Amer. J. Math., 89 (1967), pp. 363-372.

[Ab2] AвнyankaR, S. S., Singularities of algebraic curves, Analytic methods in mathematical Physics Conference Proceedings, Gordon and Breech (1970), pp. 3-14. 
[Ab3] Abhyankar, S. S., Algebraic Geometry for Scientists and Engineers(book), American Mathematical Society, Providence (1990).

[Ar] V.I. ARNOLD, Normal forms of functions in neighborhoods of degenerate critical points, Russian Math. Surveys, 29 (1974), pp. 10-50.

[Br] Brauner, K., Zur Geometrie der Funktionen Zweier Komplexen Vesänderlicken, Abh. Math. Sem. Hamburg, 6 (1928), pp. 1-54.

[Bri-Kn] Brieskorn, E. And Knorrer, H., Plane Algebraic Curves Vol.I, Vol.II, Birkhauser Verlag Basel (1986).

[Bu] Burau, W., Kennzeichung der Schlauchknoten, Abh. Math. Sem. Hamburg, 9 (1932), pp. $125-133$.

[En-Ch] EnRIQues, F And Chisini, O., Lezioni sulla teoria geometrica delle equazioni e delle funzioni algebriche, 3 Vols., Bologna, 1915, 1918, 1924.

[L] Lejeune, M., Sur l' equivalence des singularité des courbes algebroides planes, Coefficients de Newton, Centre de Math. del 1'Ecole Polytechnique (1969).

[R] ReEve, J. E., A summary of results in the topological classification of plane algebroid singularities, Rendiconti Seminario Matematico Universita e Politecnico Torino, 14 (1955), pp. 159-87.

[Z1] ZARISKI, O, On the topology of algebroid singularities, Amer. J. Math., 54 (1932), pp. 433465.

[Z2] ZARISKI, O, Studies in equisingularity I: Equivalent singularities of plane algebroid curves, Amer. J. Math., 87 (1965), pp. 507-536. 
